\title{
Consistent Fluid Property Evaluation in RELAP5-3D
}

\author{
Dr. George L Mesina
}

October, 2016

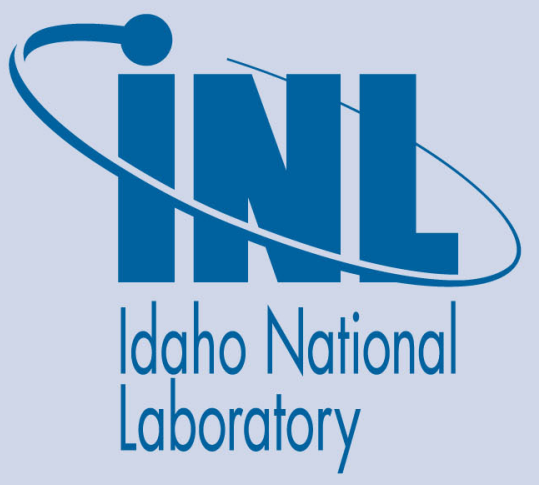

The INL is a U.S. Department of Energy National Laboratory operated by Battelle Energy Alliance 


\section{DISCLAIMER}

This information was prepared as an account of work sponsored by an agency of the U.S. Government. Neither the U.S. Government nor any agency thereof, nor any of their employees, makes any warranty, expressed or implied, or assumes any legal liability or responsibility for the accuracy, completeness, or usefulness, of any information, apparatus, product, or process disclosed, or represents that its use would not infringe privately owned rights. References herein to any specific commercial product, process, or service by trade name, trade mark, manufacturer, or otherwise, does not necessarily constitute or imply its endorsement, recommendation, or favoring by the U.S. Government or any agency thereof. The views and opinions of authors expressed herein do not necessarily state or reflect those of the U.S. Government or any agency thereof. 
INL/EXT-16-40085

\title{
Consistent Fluid Property Evaluation in RELAP5-3D
}

\author{
Dr. George L Mesina
}

October, 2016

Idaho National Laboratory Idaho Falls, Idaho 83415

Prepared for the

U.S. Department of Energy

Office of Nuclear Energy

Under DOE Idaho Operations Office

Contract DE-AC07-05ID14517 

Title: Consistent Fluid Property Evaluation in RELAP5-3D

October, 2016

Code Version: 4.3.4

Authors:

G. L. Messina Merge Medina Date: $10 / 27 / 2016$

Reviewer:

C. B. Davis CBDaris

Date: $10 / 27 / 16$

Manager:

J.R. Wolf

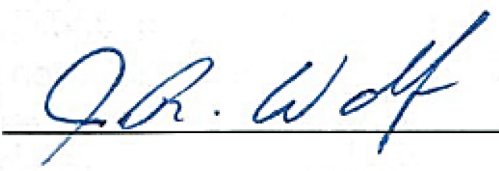

Date: 


\section{EXECUTIVE SUMMARY}

Previous investigations have developed and tested a methodology to improve the consistency between the analytical method that calculates the fluid property derivatives used by RELAP5-3D in the numerical solution scheme and the linearized solution scheme itself. An alternative numerical method is to calculate linear approximations to the fluid properties and their derivatives by interpolation for the properties and finite differencing for the derivatives. Both the analytical and numerical methods produce an approximation at a fluid state point in a bounding rectangle of the fluid property table. Further, the analytical and numerical approximations may be combined via a weighted average with a weighting factor between 0.0 and 1.0. This average has shown potential to reduce mass error relating to the fluid properties in RELAP5-3D.

This linear interpolation and weighted averaging methodology has been implemented for a fixed weighting factor in RELAP5-3D. Further, two additional options have been developed; these adjust the weighting factor in the average according to the location within the bounding rectangle. The first calculates the weighting factor based only on the position between the left and right internal energy endpoints. The second also factors the position between the upper and lower pressure grid points into the calculation of the weighting factor. The new coding has been implemented only for fluid $\mathrm{H} 2 \mathrm{ON}$. The implementation passes verification testing and expanded testing of the various options. Graphs are presented from six kinds of test cases, a two volume basic model with both insurge and outsurge, Edward's O'Brien blowdown, typical PWR, Moby Dick, and Steam Bubbling through Water. Some trends of the effect of crossing fluid property table pressure and internal energy grid line are identified and discussed. Preliminary results show that several variations of the consistent fluid methodology can reduce mass error. Based on the number of test cases in the study, there is no clear best choice. 


\section{CONTENTS}

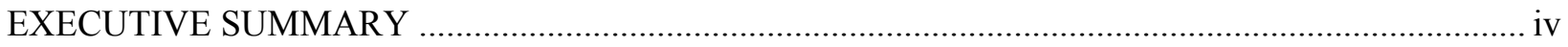

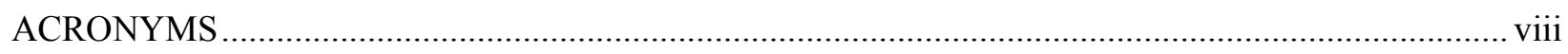

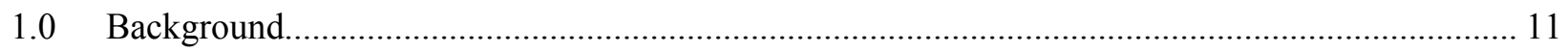

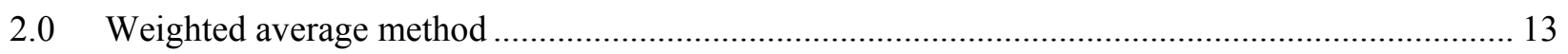

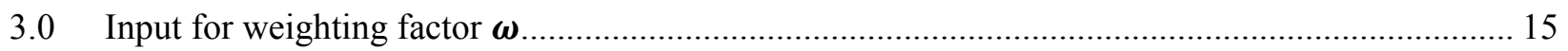

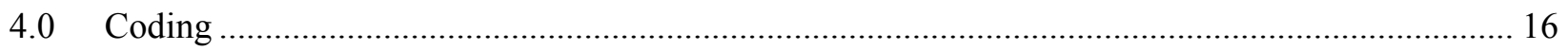

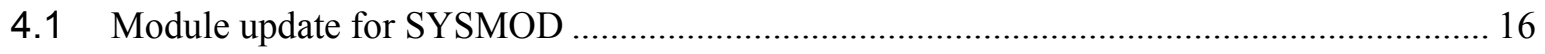

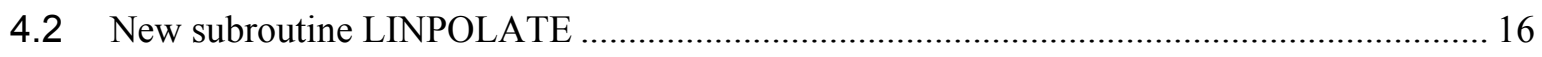

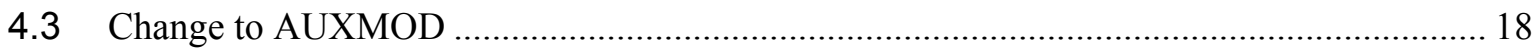

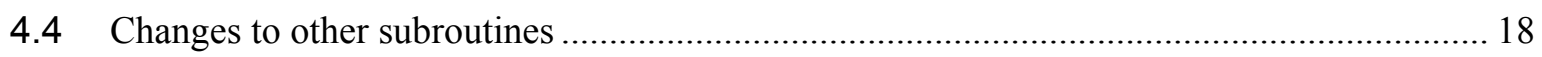

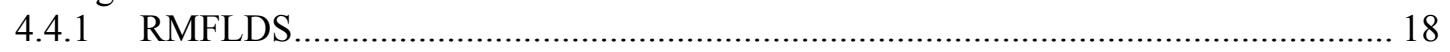

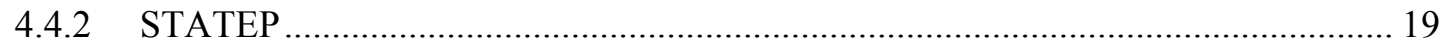

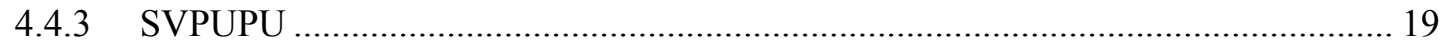

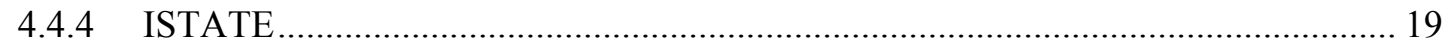

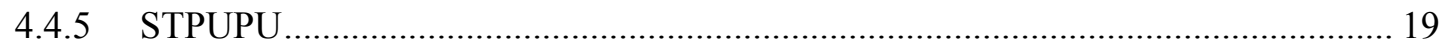

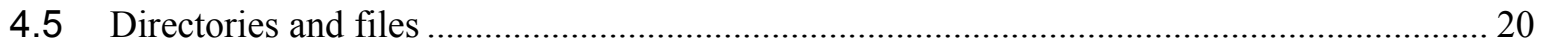

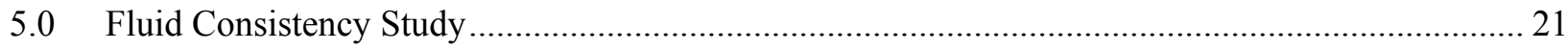

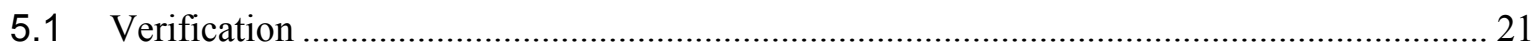

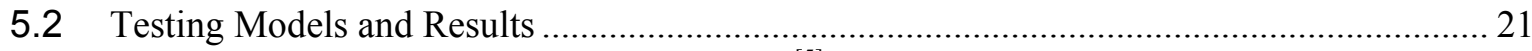

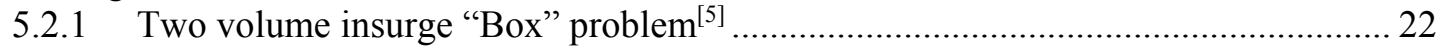

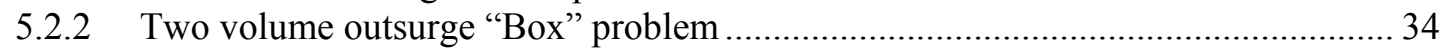

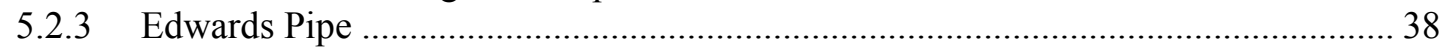

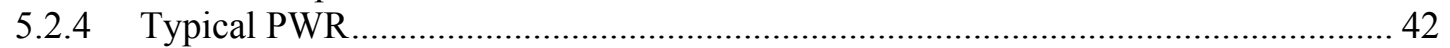

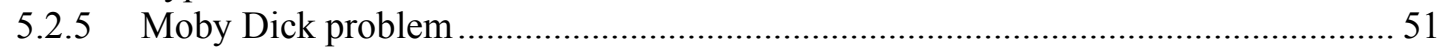

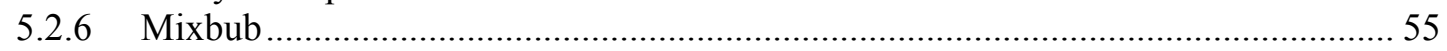

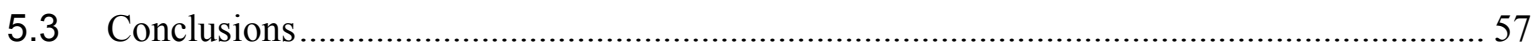

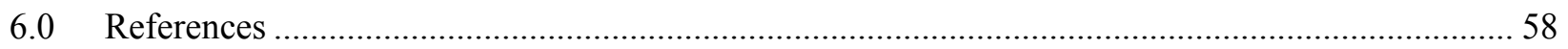




\section{FIGURES}

Figure 4.2.1 Calculation of varying Omega corresponding to input Omega $=-1$ or $02 \ldots \ldots \ldots \ldots \ldots \ldots \ldots \ldots . \ldots \ldots$

Figure 4.2.2 Statement function LINTER for linear interpolant of $\mathrm{f}(\mathrm{x}, \mathrm{y})$.......................................... 16

Figure 4.2.3 Calculation of bounding rectangle corner points \& dimensionless distances..................... 16

Figure 4.2.4 Linearly interpolate the physical quantities, $\mathrm{T}, \boldsymbol{\rho}, \mathbf{\kappa}, \mathbf{C p}, \boldsymbol{\beta}, \mathbf{s}, \mathbf{k}, \boldsymbol{\mu}$, and $\boldsymbol{v} \ldots \ldots \ldots \ldots \ldots \ldots \ldots \ldots . . . . . . . . .17$

Figure 4.2.5 Calculation of densities, enthalpies and temperatures ..................................................... 17

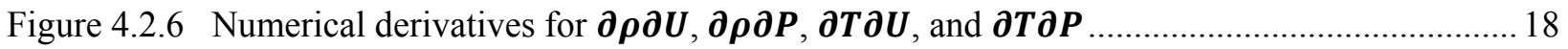

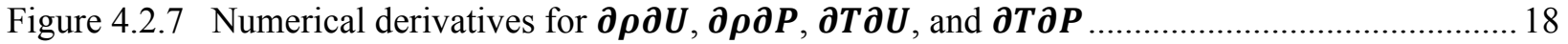

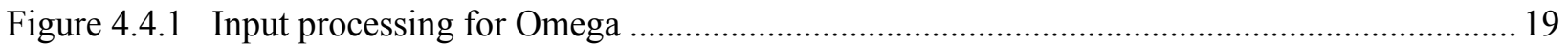

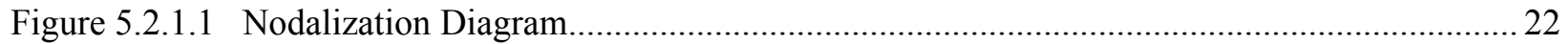

Figure 5.2.1.2 Mass Error for Box 113, all $\boldsymbol{\omega}$ excellent, except $\boldsymbol{\omega}=-2$............................................. 23

Figure 5.2.1.3a Box 7556 Mass error, $-\mathbf{1} \leq \boldsymbol{\omega}<1$ outperform default RELAP5-3D ..........................23

Figure 5.2.1.3b Box 7556 Mass error, $-\mathbf{1} \leq \boldsymbol{\omega}<1$ outperform default RELAP5-3D..........................24

Figure 5.2.1.4 Box 7495 Mass error is smaller for $\boldsymbol{\omega}=\mathbf{0 . 5}$, nearly zero for $\boldsymbol{\omega}=\mathbf{0 . 0} \mathbf{0} \ldots \ldots \ldots \ldots \ldots \ldots \ldots .24$

Figure 5.2.1.5 Box 7400 Mass error has local minima at pressure grid values. ....................................25

Figure 5.2.1.6a Mass Error for Box 5629, $\boldsymbol{\omega}=0$ excellent, $\boldsymbol{\omega}=-2$ spikes..........................................26

Figure 5.2.1.6b Zoom for Box 5629, $\boldsymbol{\omega} \neq-2$ outperform default RELAP5-3D ......................................2 26

Figure 5.2.1.7 Mass error in Box 7445, all $\boldsymbol{\omega}<1$ outperform default RELAP5-3D ............................ 27

Figure 5.2.1.8 Box 7446 Mass error: all $\boldsymbol{\omega}$ good. No u-crossing, corner at middle p-point....................2 27

Figure 5.2.1.9a Mass Error for Box 1936, all $\boldsymbol{\omega} \neq \mathbf{1} . \mathbf{0}$ better than default, $\boldsymbol{\omega}=0$ excellent.................28

Figure 5.2.1.9b Mass Error for Box 1936, all $\boldsymbol{\omega} \neq \mathbf{1} . \mathbf{0}$ better than default, $\boldsymbol{\omega}=0$ excellent................. 28

Figure 5.2.1.10 Mass Error for Box 5651, all $\boldsymbol{\omega}=0.5,1.0$ good, $\boldsymbol{\omega}=0$ good, $\boldsymbol{\omega}<\mathbf{0}$ poor. .................29

Figure 5.2.1.11 Mass error in Box 5928, $1>\boldsymbol{\omega} \geq \mathbf{0}$ outperform default RELAP5-3D .......................29

Figure 5.2.1.12 Mass error in Box 5928, $1>\boldsymbol{\omega} \geq \mathbf{- 1}$ outperform default RELAP5-3D .................... 30

Figure 5.2.1.13 Box sc1 Mass error, $\boldsymbol{\omega} \neq \mathbf{- 2}$ outperform default RELAP5-3D .................................31

Figure 5.2.1.14 Box sc2 Mass error, $\boldsymbol{\omega} \neq \mathbf{- 2}$ outperform default RELAP5-3D ................................... 31

Figure 5.2.1.15 Box sc3 Mass error, all $\boldsymbol{\omega}<\mathbf{1}$ outperform default RELAP5-3D ................................ 32

Figure 5.2.1.16 Box sc4 Mass error, $\boldsymbol{\omega}=\mathbf{- 2}$ outperform default RELAP5-3D …............................ 32

Figure 5.2.1.17 Box sc5 Mass error, $\boldsymbol{\omega} \neq \mathbf{- 2}$ outperforms default RELAP5-3D ................................. 33

Figure 5.2.1.18 Box sc6 Mass error, $\mathbf{0} \leq \boldsymbol{\omega}<\mathbf{1}$ outperforms default RELAP5-3D .............................33

Figure 5.2.1.1 Diagram of the Edward's O'Brien Blowdown test facility .......................................... 38 
Figure 5.2.2.2 Diagram of the Edward's O'Brien Blowdown test facility …......................................... 38

Figure 5.2.2.3a Edwards Pipe mass error. One U grid point crossed, INL card-1 options ...................... 39

Figure 5.2.2.3b Edwards Pipe mass error. One U grid point crossed, BTS card-1 options ..................... 39

Figure 5.2.2.4 Edwards Pipe mass error. P grid points crossed, INL card-1 options............................... 40

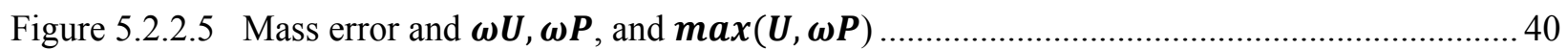

Figure 5.2.2.5 DA plot Pressure vs. time for all five omega runs........................................................... 41

Figure 5.2.2.6 DA plot Void fraction vs. time for all five omega runs.............................................. 41

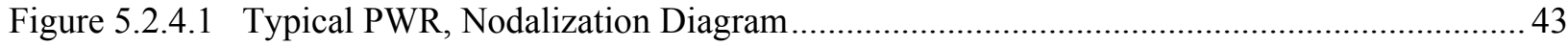

Figure 5.2.4.2a Mass error for various $\boldsymbol{\omega}$ values with $\Delta \mathrm{t}=0.1$, INL card-1 options ............................... 44

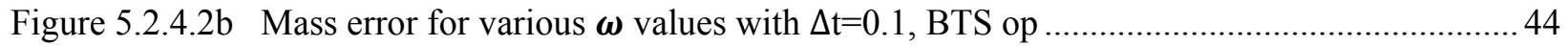

Figure 5.2.4.2c Mass error $\Delta \mathrm{t}=0.1$ and BTS options run until failure................................................. 45

Figure 5.2.4.3a Mass error for various $\boldsymbol{\omega}$ values with $\Delta \mathrm{t}=0.05$, INL card- 1 options ............................ 46

Figure 5.2.4.3b Mass error for various $\boldsymbol{\omega}$ values with $\Delta \mathrm{t}=0.05$, BTS options ...................................46

Figure 5.2.4.4a Mass error for various $\boldsymbol{\omega}$ values with $\Delta \mathrm{t}=0.01$, INL card-1 options ............................ 47

Figure 5.2.4.4b Mass error for various $\boldsymbol{\omega}$ values with $\Delta \mathrm{t}=0.01$, BTS options ...................................... 47

Figure 5.2.4.5a Mass error for various $\boldsymbol{\omega}$ values with $\Delta \mathrm{t}=0.005$, INL card-1 options .......................... 48

Figure 5.2.4.5b Mass error for various $\boldsymbol{\omega}$ values with $\Delta \mathrm{t}=0.005$, BTS options ................................... 48

Figure 5.2.4.6a Mass error for various $\boldsymbol{\omega}$ values with $\Delta \mathrm{t}=0.001$, INL card-1 options ........................... 49

Figure 5.2.4.6b Mass error for various $\boldsymbol{\omega}$ values with $\Delta \mathrm{t}=0.001$, BTS options .................................. 49

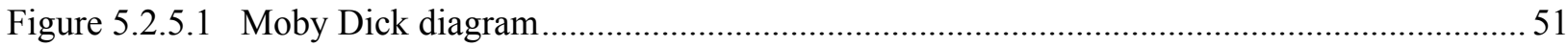

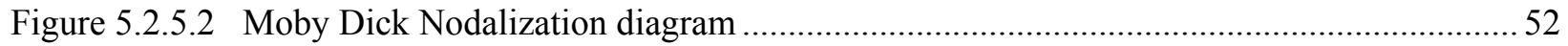

Figure 5.2.5.1 Pressure vs. elevation for Moby Dick, all five standard omega values .......................... 53

Figure 5.2.6.1 Nodalization diagram for the mixbub input model...................................................... 55

Figure 5.2.6.1a Mass error in mixbub.i for various omega values, INL card-1 options .........................5 56

Figure 5.2.6.1b Mass error in mixbub.i for various omega values, BTS card-1 options ........................56 


\section{ACRONYMS}

BC Boundary Conditions

DA Developmental Assessment

FDM Finite Difference Method

INL Idaho National Laboratory

RELAP Reactor Excursion and Leak Analysis Package

$\mathrm{V} \& \mathrm{~V} \quad$ Verification and Validation 


\section{SYMBOLS}

\section{$\underline{\text { English }}$}

$\mathrm{C}_{\mathrm{p}} \quad$ Specific heat capacity

$f / f_{\text {num }} \quad$ Function of internal energy and pressure / linear interpolant of $f$.

$\mathrm{P} \quad$ Pressure / Saturation pressure of steam / Critical pressure

$T^{s} / T_{f} / T_{g} \quad$ Temperatures: saturation/liquid/gas

$\mathrm{U} / \mathrm{U}_{\mathrm{f}} / \mathrm{U}_{\mathrm{g}} \quad$ Specific Internal Energy / Liquid specific internal energy / gas specific internal energy

$X_{\mathrm{n}} \quad$ Noncondensable Quality

$\mathrm{x} / x_{1} / x_{2} \quad$ Abscissa coordinate designation (internal energy) / left endpoint / right endpoint

$\mathrm{y} / y_{1} / y_{2}$ Ordinate coordinate designation (pressure) / bottom endpoint / top endpoint

3D Three dimensional

\section{Greek}

$\alpha(\mathrm{x}) \quad$ Linear interpolation weighting factor as a function of $\mathrm{x}$

$\alpha_{\mathrm{g}} \quad$ Volume fraction of the gas

$\beta(y) \quad$ Linear interpolation weighting factor as a function of $y$

$\beta_{f} / \beta_{g} \quad$ Coefficient of thermal expansion: liquid, gas

$\varepsilon \quad$ Small value

$\Delta \mathrm{t} \quad$ Timestep for Hydrodynamic Advancement

$\kappa_{f} / \kappa_{g} \quad$ Pressure coefficient of expansion

$\mathrm{V} \quad$ Specific volume

$\rho_{f} / \rho_{g} \quad$ Liquid / gas density

$\omega \quad$ Weighting factor between analytical and linear approximations 


\subsection{Background}

RELAP5-3D ${ }^{[1]}$ uses independent variables of pressure and specific internal energy in its numerical solution scheme. In discretizing the field equations, the densities, $\rho_{f}$ and $\rho_{g}$, and temperatures, $T^{s}, T_{f}$ and $T_{g}$, are approximated at new time by first degree Taylor Polynomials. The coefficients of these polynomials are the partial derivatives of the temperatures and densities with respect to pressure and the appropriate phasic internal energy in Volume I, Eqns. (3.1-98), (3.1-99), (3.1-102), (3.1-103), (3.1-104). These are represented here as Eqns. (1-1) through (1-5) where the derivatives are evaluated at time level $\mathrm{n}$.

$$
\begin{aligned}
& \rho_{f}^{n+1} \approx \rho_{f}^{n}+\frac{\partial \rho_{\mathrm{f}}}{\partial \mathrm{U}_{\mathrm{f}}}\left(\widetilde{U}_{f}^{n+1}-\widetilde{U}_{f}^{n}\right)+\frac{\partial \rho_{\mathrm{f}}}{\partial \mathrm{U}_{\mathrm{f}}}\left(P^{n+1}-P^{n}\right) \\
& \rho_{g}^{n+1} \approx \rho_{g}^{n}+\frac{\partial \rho_{\mathrm{g}}}{\partial \mathrm{X}_{n}}\left(\widetilde{X}_{n}^{n+1}-\widetilde{X}_{n}^{n}\right)+\frac{\partial \rho_{\mathrm{g}}}{\partial \mathrm{U}_{g}}\left(\widetilde{U}_{f}^{n+1}-\widetilde{U}_{f}^{n}\right)+\frac{\partial \rho_{\mathrm{g}}}{\partial \mathrm{P}}\left(P^{n+1}-P^{n}\right) \\
& T_{f}^{n+1} \approx T_{f}^{n}+\frac{\partial \mathrm{T}_{\mathrm{f}}}{\partial \mathrm{U}_{\mathrm{f}}}\left(\widetilde{U}_{f}^{n+1}-\widetilde{U}_{f}^{n}\right)+\frac{\partial \mathrm{T}_{\mathrm{f}}}{\partial \mathrm{U}_{\mathrm{f}}}\left(P^{n+1}-P^{n}\right) \\
& T_{g}^{n+1} \approx T_{g}^{n}+\frac{\partial \mathrm{T}_{\mathrm{g}}}{\partial \mathrm{X}_{n}}\left(\widetilde{X}_{n}^{n+1}-\widetilde{X}_{n}^{n}\right)+\frac{\partial \mathrm{T}_{\mathrm{g}}}{\partial \mathrm{U}_{g}}\left(\widetilde{U}_{g}^{n+1}-\widetilde{U}_{g}^{n}\right)+\frac{\partial \mathrm{T}_{\mathrm{g}}}{\partial \mathrm{P}}\left(P^{n+1}-P^{n}\right) \\
& T_{g}^{s, n+1} \approx T_{g}^{s, n}+\frac{\partial T^{s}}{\partial \mathrm{x}_{n}}\left(\widetilde{X}_{n}^{n+1}-\widetilde{X}_{n}^{n}\right)+\frac{\partial T^{s}}{\partial \mathrm{U}_{g}}\left(\widetilde{U}_{g}^{n+1}-\widetilde{U}_{g}^{n}\right)+\frac{\partial T^{s}}{\partial \mathrm{P}}\left(P^{n+1}-P^{n}\right)
\end{aligned}
$$

Noncondensable quality $\mathrm{X}_{n}$ is not considered in this project, therefore terms involving $\mathrm{X}_{n}$ drop out of preceding equation. In the absence of noncondensable, saturation temperature is a function of pressure alone, so Eqn. (1-5) is eliminated. The remaining derivatives are: $\frac{\partial \rho_{f}}{\partial U_{f}}, \frac{\partial \rho_{f}}{\partial P}$, and $\frac{\partial T_{f}}{\partial U_{f}}, \frac{\partial T_{f}}{\partial P}$ for liquid, and $\frac{\partial \rho_{g}}{\partial U_{g}}, \frac{\partial \rho_{g}}{\partial P}$, and $\frac{\partial T_{g}}{\partial U_{g}}, \frac{\partial T_{g}}{\partial P}$ for vapor.

The code calculates these derivatives from the following basic fluid state properties that are contained in the steam tables at grid points:

- specific volume $v$

- specific heat capacity $C_{p}$

- the isobaric coefficient of thermal expansion $\beta$

- and the isothermal coefficient of compressibility $\kappa$

The formulas for the required derivatives are given in Eqns. (3.2-5) through (3.2-8) for the liquid phase, restated here as Eqns. (1-6) through (1-9).

$$
\begin{aligned}
& \left(\frac{\partial \rho_{f}}{\partial U_{f}}\right)_{P}=\frac{v_{f} \beta_{f}}{\left(C_{p f}-v_{f} \beta_{f} P\right) v_{f}^{2}} \\
& \left(\frac{\partial \rho_{f}}{\partial P}\right)_{U_{f}}=\frac{C_{p f} v_{f} \kappa_{f}-T_{f}\left(v_{f} \beta_{f}\right)^{2}}{\left(C_{p f}-v_{f} \beta_{f} P\right) v_{f}^{2}} \\
& \left(\frac{\partial T_{f}}{\partial U_{f}}\right)_{P}=\frac{1}{C_{p f}-v_{f} \beta_{f} P} \\
& \left(\frac{\partial T_{f}}{\partial P}\right)_{U_{f}}=\frac{P v_{f} \kappa_{f}-T_{f} v_{f} \beta_{f}}{C_{p f}-v_{f} \beta_{f} P}
\end{aligned}
$$

The manual states that the gas phase equations are identical with the subscript "f" for liquid replaced by " $g$ " for gas. 
The steam table properties at non-grid points are determined by various interpolations, some of which are linear and some of which are not. The interpolated specific volume, specific heat capacity, etc. are then used to obtain interpolated values for the four derivatives.

A previous project ${ }^{[2]}$ evaluated a different interpolation scheme. It calculates all properties via linear interpolations of the values at the grid points. Thereafter it calculates the values of the eight derivatives needed in Eqns. (1-1) through (1-4) from the interpolated values of densities, temperatures, specific internal energies, and pressure at the four grid points. This latter method is referred to as a numerical derivative scheme because the four derivatives can be calculated directly from the known values of $\rho$ and $T$ at the grid points.

Specifically, the grid is contained in the TPF-file which tabulated values of pressure, and liquid and gas internal energies, $v, C_{p}, \beta, \kappa$, and temperatures. Let function $\mathrm{f}(\mathrm{x}, \mathrm{y})$ represent one of the functions $\mathrm{T}_{\mathrm{f}}, \mathrm{T}_{\mathrm{g}}, \rho_{\mathrm{f}}$, or $\rho_{\mathrm{g}}$, whose derivatives are sought, and let $\mathrm{x}$ denote internal energy and $\mathrm{y}$ denote pressure. The bounding grid points for specific point $(\mathrm{x}, \mathrm{y})$ satisfy $x_{1} \leq x \leq x_{2}$ and $y_{1} \leq y \leq y_{2}$. Define dimensionless distances $\alpha$ and $\beta$ by Eqns. (1-10) and (1-11), so that Eqn. (1-12) defines the linear interpolant $F(x, y)$ for $f(x, y)$ in the bounding rectangle.

$$
\begin{aligned}
& \alpha(x)=\frac{x-x_{1}}{x_{2}-x_{1}} \\
& \beta(y)=\frac{y-y_{1}}{y_{2}-y_{1}} \\
& f_{\text {num }}(x, y)=(1-\alpha)(1-\beta) f\left(x_{1}, y_{1}\right)+\beta(1-\alpha) f\left(x_{1}, y_{2}\right) \\
&+\alpha(1-\beta) f\left(x_{2}, y_{1}\right)+\beta \alpha f\left(x_{2}, y_{2}\right)
\end{aligned}
$$

Thus, $\alpha\left(x_{1}\right)=0, \alpha\left(x_{2}\right)=1, \beta\left(y_{1}\right)=0$, and $\beta\left(y_{2}\right)=1$, so that $f_{\text {num }}\left(x_{i}, y_{j}\right)=f\left(x_{i}, y_{j}\right)$ for $\mathrm{i}=1,2$ and $\mathrm{j}=1,2$. Since $1-\alpha=\frac{x_{2}-x}{x_{2}-x_{1}}$ and $1-\beta=\frac{y_{2}-y}{y_{2}-y_{1}}$, the derivatives are given by,

$$
\begin{aligned}
\left(\frac{\partial f}{\partial x}\right)_{n u m} & =\frac{d \alpha}{d x}\left\{-(1-\beta) f\left(x_{1}, y_{1}\right)-\beta f\left(x_{1}, y_{2}\right)+(1-\beta) f\left(x_{2}, y_{1}\right)+\beta f\left(x_{2}, y_{2}\right)\right\} \\
& =\frac{1}{x_{2}-x_{1}}\left\{(1-\beta) f\left(x_{2}, y_{1}\right)-(1-\beta) f\left(x_{1}, y_{1}\right)+\beta f\left(x_{2}, y_{2}\right)-\beta f\left(x_{1}, y_{2}\right)\right\} \\
& =\left\{(1-\beta)\left[f\left(x_{2}, y_{1}\right)-f\left(x_{1}, y_{1}\right)\right]+\beta\left[f\left(x_{2}, y_{2}\right)-f\left(x_{1}, y_{2}\right)\right]\right\} /\left(x_{2}-x_{1}\right) \\
\left(\frac{\partial f}{\partial y}\right)_{\text {num }} & =\frac{d \beta}{d y}\left\{-(1-\alpha) f\left(x_{1}, y_{1}\right)-\alpha f\left(x_{2}, y_{1}\right)+(1-\alpha) f\left(x_{1}, y_{2}\right)+\alpha f\left(x_{2}, y_{2}\right)\right\} \\
& =\left\{(1-\alpha)\left[f\left(x_{1}, y_{2}\right)-f\left(x_{1}, y_{1}\right)\right]+\alpha\left[f\left(x_{2}, y_{2}\right)-f\left(x_{2}, y_{1}\right)\right]\right\} /\left(y_{2}-y_{1}\right)
\end{aligned}
$$

The numerical derivatives are linear and therefore only first order accurate while the code applies approximations to analytical forms that have higher order of accuracy. Moreover, the piecewise linear interpolant given by Eqns. $(1-10)$ to $(1-12)$ has discontinuities at the grid edges. A smoothing method is given in Sec. 2. Its input is explained in Sec. 3. Implementation of the linear interpolant and numerical derivatives of Eqns. (1-12) to (1-14) is explained in Sec. 4. Sample problems and their mass error graphs are discussed in Sec. 5.

All implementation and testing for this method is confined to the fluid $\mathrm{H} 2 \mathrm{ON}$. 


\subsection{Weighted average method}

The numerical derivatives of Eqns. (1-13) and (1-14) are linear and thus more consistent with other linear schemes in RELAP5-3D. Therefore, using the numerical derivatives could result in reduced mass error than the continuous derivatives. A small 2 volume test model was constructed to test this. The results from the previous project showed that the mass error was significantly lower than when Eqns. (1-6) through (1-9) are used. Improved coding from this project, shown in Sec. 5, agrees with this result. However, for more complicated models shown in Sec. 5 such as Edwards Pipe, this is not always true. One reason is discontinuity at the edges of grid rectangles.

Eqns. (1-13) and (1-14) are piecewise linear with jump discontinuities at grid rectangle edges. For example, because $\beta\left(y_{2}\right)=\left(y_{2}-y_{1}\right) /\left(y_{2}-y_{1}\right)=1$ at $y=y_{2}$ in the left rectangle and at $y=y_{2}$ in the right rectangle, $\beta\left(y_{2}\right)=\left(y_{2}-y_{2}\right) /\left(y_{3}-y_{2}\right)=0$,

$$
\begin{aligned}
& \left(\frac{\partial f}{\partial x}\right)_{n u m}\left(x_{2}-\varepsilon, y_{2}\right)=1\left[f\left(x_{2}, y_{2}\right)-f\left(x_{1}, y_{2}\right)\right] /\left(x_{2}-x_{1}\right) \\
& \left(\frac{\partial f}{\partial x}\right)_{n u m}\left(x_{2}+\varepsilon, y_{2}\right)=(1-0)\left[f\left(x_{3}, y_{2}\right)-f\left(x_{2}, y_{2}\right)\right] /\left(x_{3}-x_{2}\right)
\end{aligned}
$$

Letting $\varepsilon \rightarrow 0$ in Eqns. (2-1) and (2-2) shows that the left-hand and right-hand derivatives of $F$ w.r.t. $x$ are unequal except in special circumstances.

Jump discontinuities are unphysical in these derivatives and produce unphysical jump discontinuities in density and temperature in Eqns. (1-1) through (1-4). On the other hand, Eqns. (1-6) through (1-9) are evaluated with fluid properties obtained by linear interpolation at points $(x, y)$ in the grid. Each of these properties is therefore continuous in $(x, y)$, therefore the resulting derivatives are also continuous. Continuity is the reason Eqns. (1-6) through (1-9) were selected for the derivative calculations over Eqns. (1-13) and (1-14).

Since both schemes have advantages and disadvantages, investigations into a means to apply both for reducing mass error were conducted in both the previous project and this one. A weighting factor, $\omega$, combines the two calculations via Eqns. (2-3), (2-4) and (2-5).

$$
\begin{aligned}
& f_{\omega}(x, y)=\omega f(x, y)+(1-\omega) f_{\text {num }}(x, y) \\
& \left(\frac{\partial F}{\partial x}\right)_{\omega}=\omega \frac{\partial f}{\partial x}(x, y)+(1-\omega)\left(\frac{\partial f}{\partial x}(x, y)\right)_{\text {num }} \\
& \left(\frac{\partial F}{\partial y}\right)_{\omega}=\omega \frac{\partial f}{\partial y}(x, y)+(1-\omega)\left(\frac{\partial f}{\partial y}(x, y)\right)_{\text {num }}
\end{aligned}
$$

Here $0 \leq \omega \leq 1$. For $\omega=1$, the calculation produces the original RELAP5-3D calculations while for $\omega=0$, the calculation is fully linear. For values in between, the code has greater consistency with the code's other linear schemes than Eqns. $(1-6,7,8,9)$ and smaller jump discontinuity than in the derivatives of Eqns. (1-13) and (1-14).

When performing the calculations, $\omega$ may be a fixed constant or variable. Varying $\omega$ from 1 at the rectangle edges to 0 inside guarantees continuous derivatives. Many ways to vary omega exist. Eqns. (2-6), (2-7), and (2-8) are simple forms that were implemented and tested in the code. 


$$
\begin{aligned}
& \omega=\frac{2\left|x-\left(x_{2}+x_{1}\right) / 2\right|}{x_{2}-x_{1}}=\frac{\left|2 x-\left(x_{2}+x_{1}\right)\right|}{x_{2}-x_{1}} \\
& \omega=\max \left(\frac{\left|2 x-\left(x_{2}+x_{1}\right)\right|}{x_{2}-x_{1}}, \frac{\left|2 y-\left(y_{2}+y_{1}\right)\right|}{y_{2}-y_{1}}\right) \\
& \omega=\min \left(\frac{\left|2 x-\left(x_{2}+x_{1}\right)\right|}{x_{2}-x_{1}}, \frac{\left|2 y-\left(y_{2}+y_{1}\right)\right|}{y_{2}-y_{1}}\right)
\end{aligned}
$$

Eqn. (2-6) has a V-shape with the minimum at the center of the grid along the $x$-axis while the cross-section of Eqn. (2-7) is V-shaped in both coordinate directions. Eqn. (2-6) considers only crossings in the $\mathrm{x}$-direction (internal energy) and ignores the $y$-direction (pressure). On the other hand, Eqn. (2-7) considers both. Many other shapes are possible, but are beyond the scope of this project. Eqn. (2-8) was programmed and tested. It produced much larger mass errors than Eqn. (2-7) for the insurge and outsurge test models and was therefore abandoned.

The application of the weighting factor has other considerations besides the shape. Each hydrodynamic system should be allowed to have its own fixed value or varying calculation of $\omega$. Moreover, a default value must be chosen. It should be $\omega=1$ to allow legacy calculations to proceed without changes to input decks.

The input of the weighting factor is presented in Sec. 3. 


\subsection{Input for weighting factor $\omega$}

So that different omega factors could be applied to different systems, the input was attached to the RELAP5-3D hydrodynamic system cards. This allows application of different $\omega$ values to different systems, even if the fluid is the same.

The default value is 1.0 for $\omega$. Any value from 0.0 to 1.0 may be input for a fixed-value of the weighting factor. Key values of -1.0 and -2.0 are also allowed and signal the code to use Eqns. (2-6) and (2-7) respectively.

The section documents the additions to the description in the Vol. 2, Appendix $A^{[3]}$ of Sec. 2.14, hydrodynamic system control cards 120 through 129 .

In Sec. 2.14.1, "Hydrodynamic System Card for Fixed Problem," add word 6 to provide the value of $\omega$ or the flag that indicates the method for varying it.

W6(R) Optional weighting factor, $\omega$, used with $\mathrm{H} 2 \mathrm{ON}$ only for averaging fluid properties and their derivatives between the original analytical calculations from Vol.1, Eqns. (3.2-5) through (3.2-8) and linear interpolants on the grid points of the fluid property file. The analytical form corresponds to $\omega=1.0$, the pure linear form corresponds to $\omega=0.0$. For $0.0<\omega<1.0$, a weighted average of the two forms is applied. The value $\omega=1.0$ is applied for all other fluids and is the default for $\mathrm{H} 2 \mathrm{ON}$.

The special values $\omega=-1.0$ and $\omega=-2.0$ signal the code to vary $\omega$ w.r.t. $U$ and $P$, instead of using a fixed value. For $\omega=-1.0$, the code varies $\omega$ from 1.0 at the left edge to the bounding rectangle to 0.0 in the middle to 1.0 at the right edge. For $\omega=-2.0$, the code varies $\omega$ from 1.0 at the left and bottom edges to the bounding rectangle to 0.0 at the midpoint of the rectangle to 1.0 at the right and top edges.

In Sec. 2.14.2, "Hydrodynamic System Card for Moving Problem," add word 8 to provide the value of $\omega$ or the flag that indicates the method for varying it.

W8(R) Optional weighting factor, $\omega$, used with $\mathrm{H} 2 \mathrm{ON}$ only for averaging fluid properties and their derivatives between the original analytical calculations from Vol.1, Eqns. (3.2-5) through (3.2-8) and linear interpolants on the grid points of the fluid property file. The analytical form corresponds to $\omega=1.0$, the pure linear form corresponds to $\omega=0.0$. For $0.0<\omega<1.0$, a weighted average of the two forms is applied. The value $\omega=1.0$ is applied for all other fluids and is the default for $\mathrm{H} 2 \mathrm{ON}$.

The special values $\omega=-1.0$ and $\omega=-2.0$ signal the code to vary $\omega$ w.r.t. $U$ and $P$, instead of using a fixed value. For $\omega=-1.0$, the code varies $\omega$ from 1.0 at the left edge to the bounding rectangle to 0.0 in the middle to 1.0 at the right edge. For $\omega=-2.0$, the code varies $\omega$ from 1.0 at the left and bottom edges to the bounding rectangle to 0.0 at the midpoint of the rectangle to 1.0 at the right and top edges. 


\subsection{Coding}

\subsection{Module update for SYSMOD}

Since each hydrodynamic system can its own value of $\omega$, the natural place to include the coding is SYSMOD as a component to the array SYS. The value of the parameter that indexes SYS is MISSYS. This is located in CTRLMOD. Thus every subroutine that accesses the weighting factor must "use" both SYSMOD and CTRLMOD. Currently, this affects only new subroutine LINPOLATE and RMFLDS, the routine that reads and processes the 120-129 cards.

\subsection{New subroutine LINPOLATE}

Subroutine LINPOLATE copies the weighting factor $\omega$ from SYSMOD into a local variable. If it holds one of the key values, -1.0 or -2.0 , the calculation in Figure 4.2.1 is undertaken.

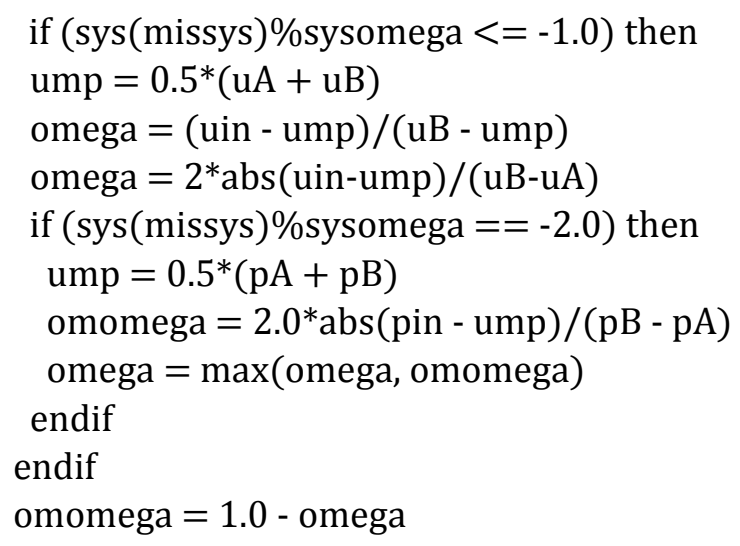

Figure 4.2.1 Calculation of varying Omega corresponding to input Omega = -1 or 02 .

Statement function LINTER, shown in Fig. 4.2.2, implements Eqn. (1-12). Here "a" stands for $\alpha$, "b" for $\beta$, f1 for $f\left(x_{1}, y_{1}\right)$, f2 for $f\left(x_{1}, y_{2}\right)$, f3 for $f\left(x_{2}, y_{1}\right)$, and f4 for $f\left(x_{2}, y_{2}\right)$.

$$
\begin{aligned}
& \text { ! Statement function } \\
& \text { ! } \mathrm{F}(\mathrm{x}, \mathrm{y})=(1-\mathrm{a})(1-\mathrm{b}) \mathrm{f}\left(\mathrm{x}_{1}, \mathrm{y}_{1}\right)+\mathrm{b}(1-\mathrm{a}) \mathrm{f}\left(\mathrm{x}_{1}, \mathrm{y}_{2}\right)+\mathrm{a}(1-\mathrm{b}) \mathrm{f}\left(\mathrm{x}_{2}, \mathrm{y}_{1}\right)+\mathrm{b} * \mathrm{a} * \mathrm{f}\left(\mathrm{x}_{2}, \mathrm{y}_{2}\right) \\
& \quad \text { real :: linter, } \mathrm{a}, \mathrm{b}, \mathrm{f} 1, \mathrm{f} 2, \mathrm{f} 3, \mathrm{f} 4 \\
& \quad \text { linter(f1, f2, f3, f4,a,b) }=\mathrm{f} 1 *(1.0-\mathrm{a}) *(1.0-\mathrm{b})+\mathrm{f} 2 * \mathrm{~b}^{*}(1.0-\mathrm{a})+\mathrm{f} 3 *(1.0-\mathrm{b}) * \mathrm{a}+\mathrm{f} 4 * \mathrm{a}^{*} \mathrm{~b}
\end{aligned}
$$

\section{Figure 4.2.2 Statement function LINTER for linear interpolant of $f(x, y)$}

Fig. 4.2.3 shows the mnemonic names for the $U$ and $P$ grid points of the surrounding rectangle. $X$ and $Y$ are the dimensionless lengths for internal energy and pressure from Eqns. $(1-10,11)$.

$$
\begin{aligned}
& \text { ! U starts at mpt1, P starts at mpt2. Grid pt. } x 1 \text { is at index ix-1, x2 at ix, y1 at iy-1, y2 at iy. } \\
& u A=a(m p t 1+i x-1) \\
& \mathrm{uB}=\mathrm{a}(\mathrm{mpt} 1+\mathrm{ix}) \\
& p A=a(m p t 2+i y-1) \\
& \mathrm{pB}=\mathrm{a}(\mathrm{mpt} 2+\mathrm{iy}) \\
& X=(s(4, n)-u A)) /(u B-u A) \\
& Y=(s(2, n)-p B) /(p A-p B)
\end{aligned}
$$

Figure 4.2.3 Calculation of bounding rectangle corner points \& dimensionless distances 
In a TPF-file, the table of fluid properties holds one property per column; however the table is stored as a linear array. Thus for a given bounding corner point, the table index of the table row plus the appropriate column index accesses the property value at the point. Calculating the corner point indices just once (ndx1, ndx2, ndx3, ndx4) and then applying the appropriate offset for each property (column number from 0 to 7 ) reduced computations. As shown in Fig. 4.2.4, this simplification, combined with statement function linter, increases readability.

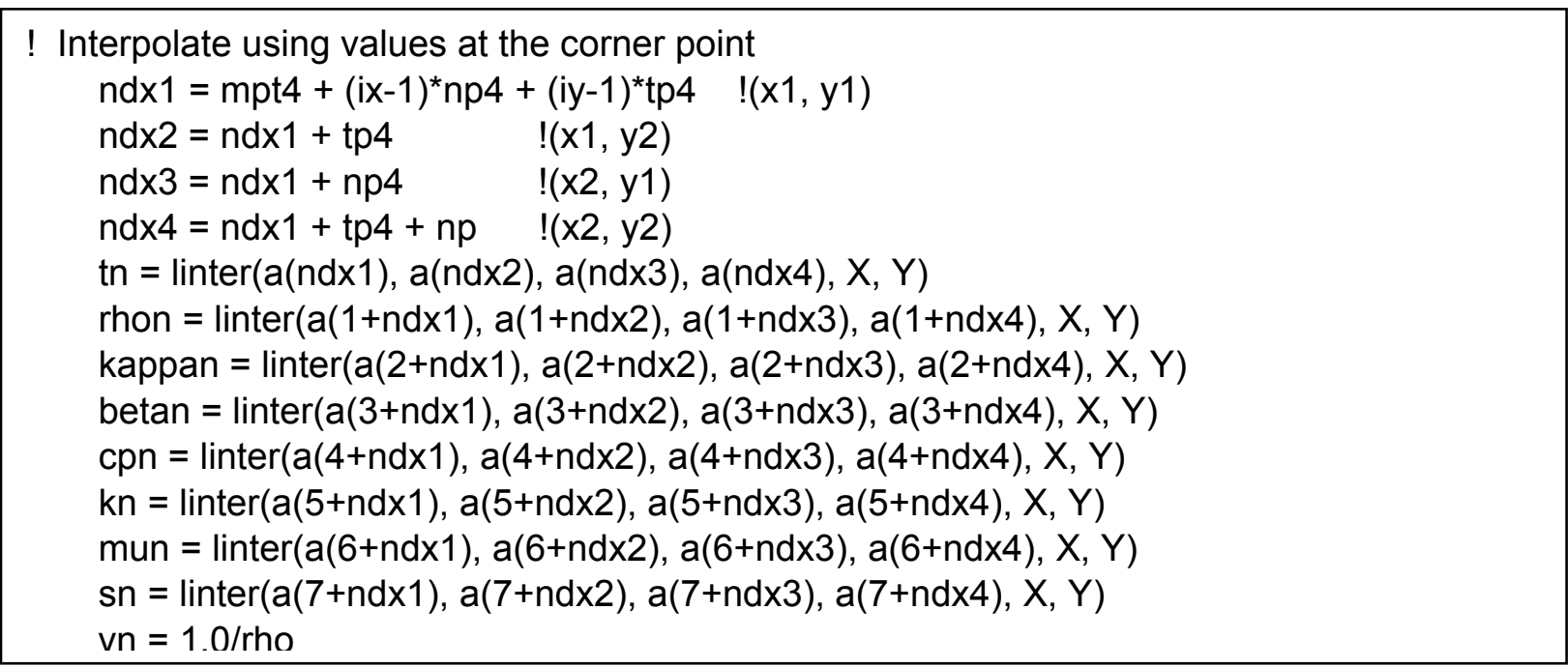

Figure 4.2.4 Linearly interpolate the physical quantities, $T, \rho, \kappa, C_{p}, \beta, s, k, \mu$, and $v$.

To calculate the derivatives with respect to enthalpy and temperature, these properties must be linearly interpolated first, as presented in Fig. 4.2.5.

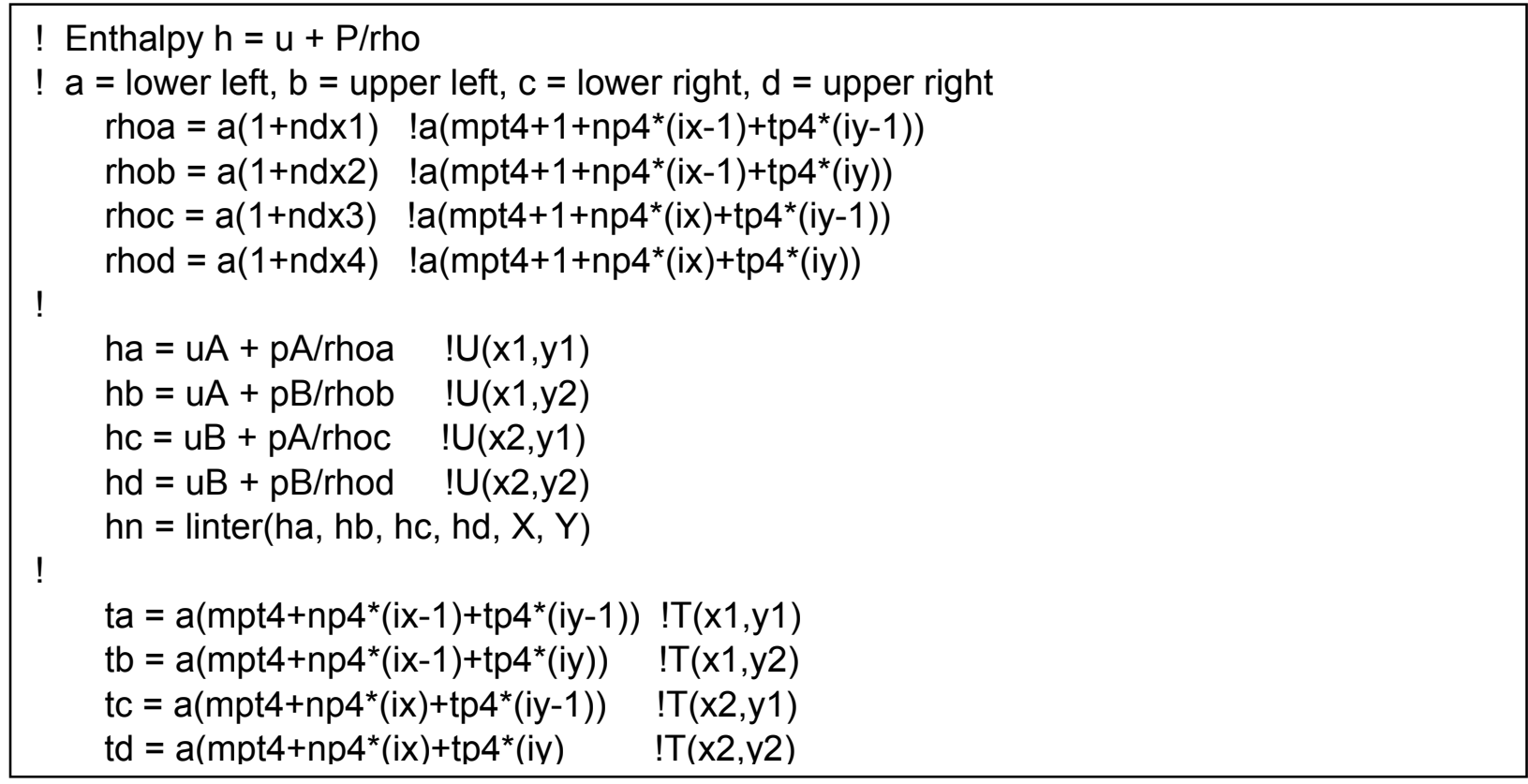

Figure 4.2.5 Calculation of densities, enthalpies and temperatures

Equations (1-13) and (1-14) are used to calculate the numerical derivatives, Fig. 4.2.6. 


$$
\begin{aligned}
& \text { ! Numerical Derivatives } \\
& !\left\{(1-\beta)\left[f\left(x_{2}, y_{1}\right)-f\left(x_{1}, y_{1}\right)\right]+\beta\left[f\left(x_{2}, y_{2}\right)-f\left(x_{1}, y_{2}\right)\right]\right\} /\left(x_{2}-x_{1}\right) \\
& \text { ! }\left\{(1-\alpha)\left[\mathrm{f}\left(\mathrm{x}_{1}, \mathrm{y}_{2}\right)-\mathrm{f}\left(\mathrm{x}_{1}, \mathrm{y}_{1}\right)\right]+\alpha\left[\mathrm{f}\left(\mathrm{x}_{2}, \mathrm{y}_{2}\right)-\mathrm{f}\left(\mathrm{x}_{2}, \mathrm{y}_{1}\right)\right]\right\} /\left(\mathrm{y}_{2}-\mathrm{y}_{1}\right) \\
& \text { drdun }=\left((\text { rhod }- \text { rhob })^{*} Y+(\text { rhoc }- \text { rhoa })^{*}(1.0-Y)\right) /(u B-u A) \\
& \text { dtdun }=\left((\text { td }-t b)^{*} Y+(\text { tc }- \text { ta })^{\star}(1.0-Y)\right) /(u B-u A) \\
& \text { drdpn }=\left((\text { rhod }- \text { rhoc })^{*} X+(\text { rhob }- \text { rhoa })^{*}(1.0-X)\right) /(p B-p A) \\
& \text { dtdpn }=\left((\mathrm{td}-\mathrm{tc})^{\star} \mathrm{X}+(\mathrm{tb}-\mathrm{ta})^{*}(1.0-\mathrm{X})\right) /(\mathrm{pB}-\mathrm{pA}
\end{aligned}
$$

Figure 4.2.6 Numerical derivatives for $\partial \rho / \partial U, \partial \rho / \partial P, \partial T / \partial U$, and $\partial T / \partial P$

With the fluid properties and their derivatives calculated, the weighted average can be formulated as shown in Fig. 4.2.7. The array, s1, transmits the derivatives from LINPOLATE to the subroutines that use them, STATEP and ISTATE, through module AUXMOD where it is declared as TT1_, a two dimensional auxiliary array.

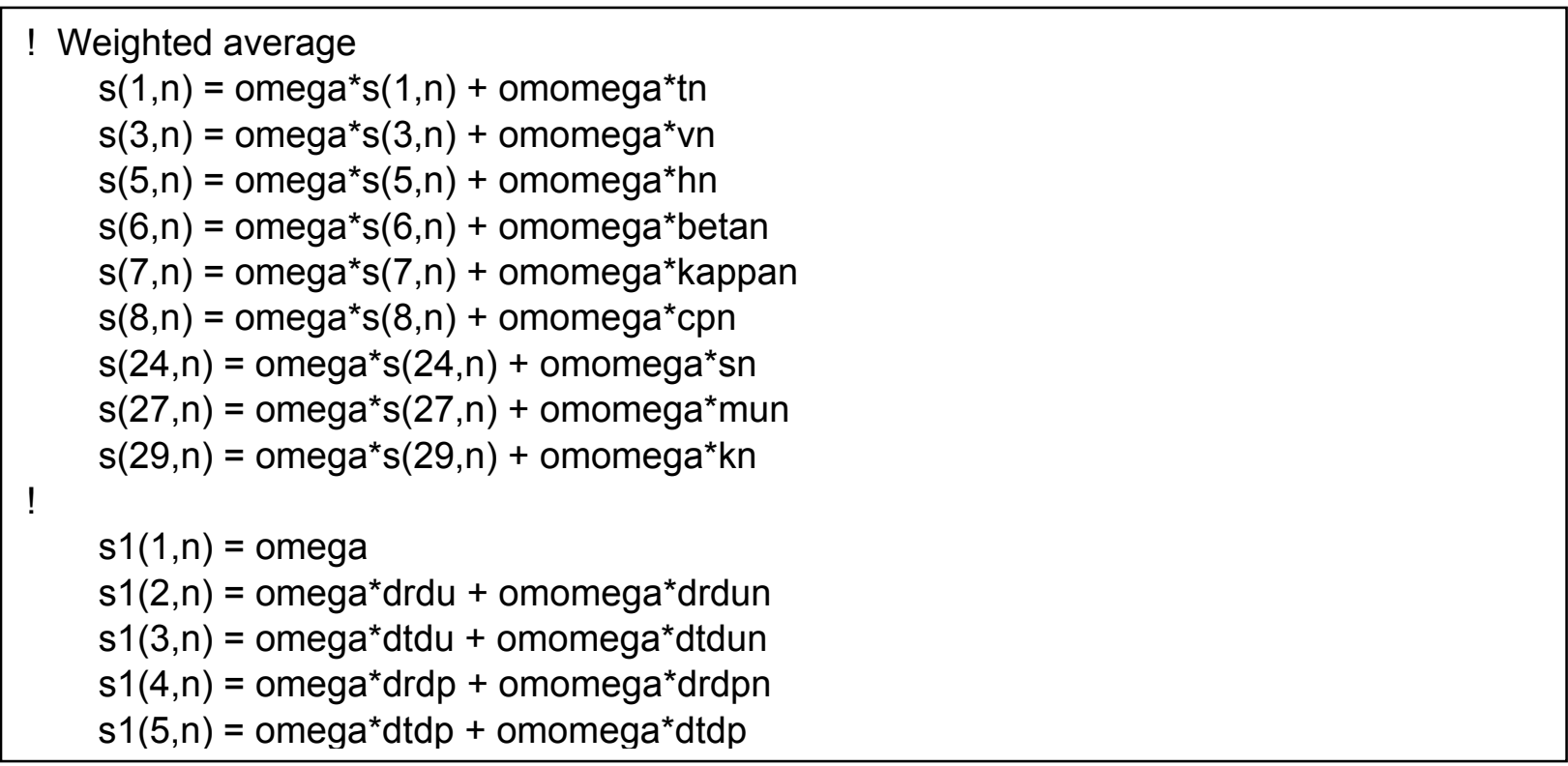

Figure 4.2.7 Numerical derivatives for $\partial \rho / \partial U, \partial \rho / \partial P, \partial T / \partial U$, and $\partial T / \partial P$

Subroutine LINPOLATE makes many declarations, calculates the analytical derivatives, and has many conditional diagnostics. The full subroutine is not presented here.

\subsection{Change to AUXMOD}

Module AUXMOD was modified to incorporate TT1_, a two dimensional auxiliary array, where both dimensions must be set with allocation. Allocate and Deallocate statements are included for the new array.

\subsection{Changes to other subroutines}

\subsubsection{RMFLDS}

This subroutine processes the $120-129$ system cards. One word was added to the card, word 6 for fixed problems and word 8 for moving problems, as documented in Sec. 3 for Vol. 2, Appendix $A$ of the input manual. Most of the coding is given in Fig. 4.4.1. If the fluid is anything 
other than $\mathrm{H} 2 \mathrm{ON}$, a nonzero value of $\omega$ is an error. For $\mathrm{H} 2 \mathrm{ON}$, allowable values $\omega=-1, \omega=$ -2 , and $0 \leq \omega \leq 1$ ) are accepted. All other values are reset to 1.0 without causing code failure but with a warning message.

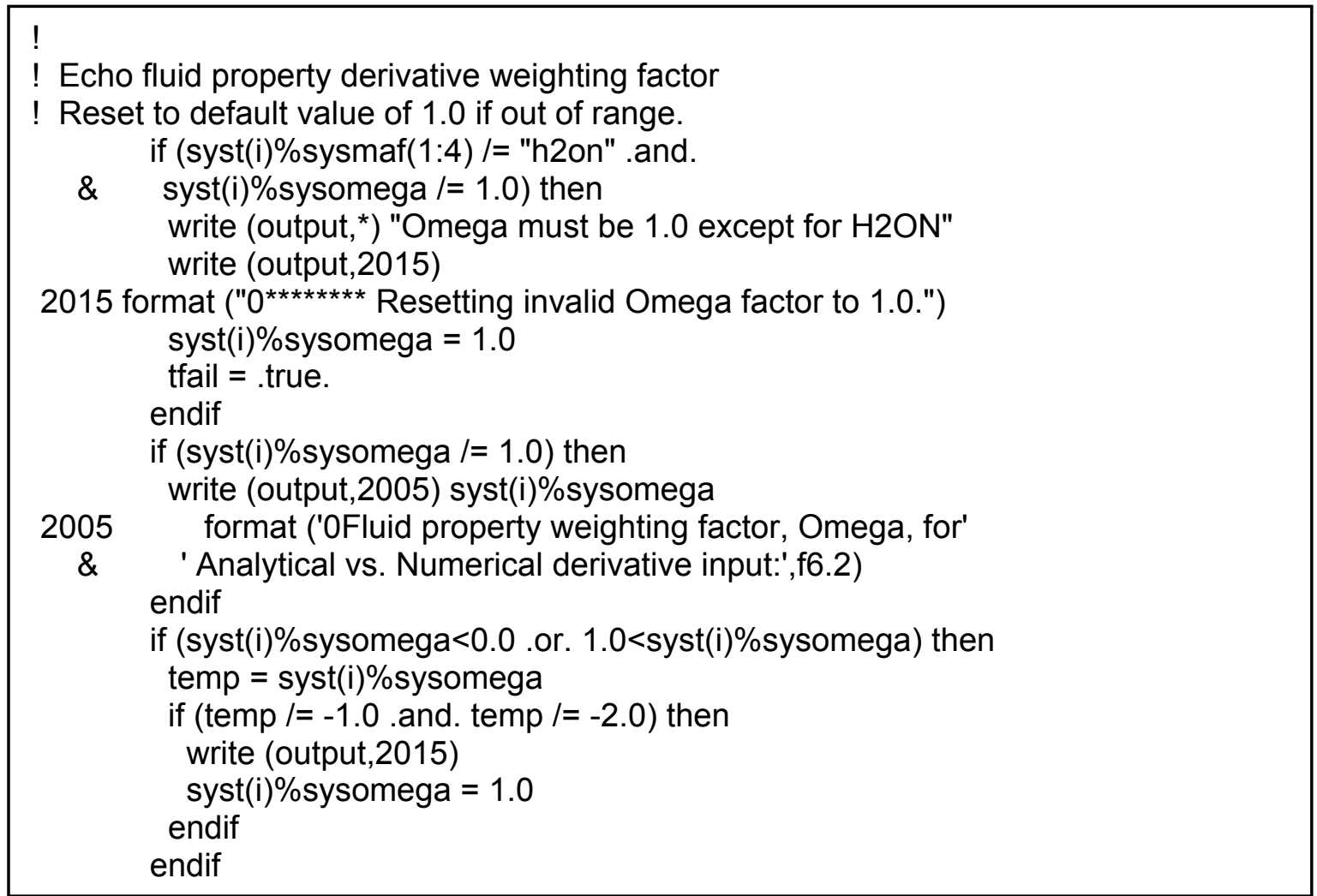

Figure 4.4.1 Input processing for Omega

\subsubsection{STATEP}

This subroutine declares the arrays s(36) and s1(6) and passes them to the GETSTATE routines that in turn call SVPUPU. Note that s1 is another name for TT1_ of AUXMOD. It also uses the weighted average approximations, instead of the usual ones, for $\mathrm{H} 2 \mathrm{ON}$ only.

\subsubsection{SVPUPU}

This subroutine uses a vector loop to calculate the analytical fluid properties and analytical derivatives for $\mathrm{H} 2 \mathrm{ON}$. It calls LINPOLATE to calculate the linear fluid properties and derivatives and also populate the $\mathrm{s} 1$ array with the derivative values.

\subsubsection{ISTATE}

This subroutine now declares the arrays s(36) and s1(6) and adds those as arguments to STPUPU through either GETSTATE routines or direct calls. It also sets MISSYS, which is passed through SYSMOD to LINPOLATE. It also uses the weighted average approximations, instead of the usual ones, for $\mathrm{H} 2 \mathrm{ON}$ only.

\subsubsection{STPUPU}

This subroutine calculates the analytical fluid properties and analytical derivatives for $\mathrm{H} 2 \mathrm{ON}$ for a single control volume. It calls LINPOLATE to calculate the linear fluid properties and derivatives and also populate the s1 array with the derivative values. 


\subsection{Directories and files}

Many auxiliary files were created for this project and stored in a project directory on the INL HPCC. The project directory is named CONFLU. It has the following subdirectories

- envrl - Environmental source files: linpolate.F90, stpupu.F, svpupu.F

- $\quad$ input - Input files and subdirectories of input files: edconflu.i, mixbub.i

- Insurge - 5 input files, one for each omega value

- Moby - 18 input deck from the Developmental Assessment suite

- Outsurge -5 input files, one for each omega value

- Typ - 5 input files, one for each omega value

- $\quad$ output - some sample output for possible future use

- relap - source files from the relap directory: istate.F, rmflds.F, statep.F, sysmod.F

- $\quad$ scripts - shell scripts and APTPLOT scripts

The scripts created a level of reproducibility. On a compatible platform, the results will be the same for the sample output stored in the output directory. Changes due to the evolution in the computer industry may cause the calculations to differ, but the scripts provide a means to adapt the work to future environments..

These include APT plot scripts, shell scripts for generating input files and running RELAP5-3D with them, and some JPEG files. The naming convention is as follows:

- doplot_ID APTPLOT scripts for the plot indicated by the ID. E.G. doplot_b131

- do_run_KIND Run the set of input problems of type KIND. E.G. dorun_typ

- doplots_KIND run many doplot_ID files to create a collection of JPEG files of one kind. There are 76 doplot_ID scripts, 7 doplots_KIND files, and 9 dorun_KIND files. 


\subsection{Fluid Consistency Study}

\subsection{Verification}

Verification requires the entire verification test suite ${ }^{[4]}$ to produce identical calculations with the current released code, 4.3.4t, and the same version with the linear interpolation update. The verification suite has no reference to omega, so the default value of $\omega=1.0$ is used.

This test was performed before and after the coding was incorporated into the preliminary version of the next code version. It passed both tests.

\subsection{Testing Models and Results}

This section shows the calculations produced with various options of $\omega$, namely $1.0,0.5$, $0.0,-1.0$, and -2.0 . The latter two values signal the code to recalculate $\omega$ according to Eqns. (2$6)$ and (2-7) on every call to LINPOLATE.

The Edward's pipe, Typical PWR, insurge and outsurge problems were studied in Ref 5 and they are repeated here to help verify that the new coding produces similar results to the original experimental coding. Note that the experimental coding did not have the numerical interpolant nor did it have the weighting function for initialization in ISTATE, therefore there are some differences between it and the new implementation.

In response to discussions at the International RELAP5 User Group (IRUG) meeting, additional runs, graphs, and tables were made. These include the 160 Moby Dick runs of Sec. 5.2.5 and runs to further study Typical PWR, Sec. 5.2.4, and Edwards Pipe, Sec. 5.2.3. In all these cases and in Sec. 5.2.6 runs were made with the BTS card-1 options.

The BTS card-1 options are: 29, 41, 54, and 55. From Appendix A of RELAP5-3D Vol. 2, these have the following meaning:

Table 5.2.1 BTS card-1 options

\begin{tabular}{|c|l|}
\hline $\begin{array}{c}\text { Card-1 } \\
\text { Option }\end{array}$ & Description \\
\hline 29 & This option accurately solves the momentum equations at low velocities. \\
\hline 41 & $\begin{array}{l}\text { This option includes energy dissipation due to form loss (code calculated abrupt } \\
\text { area change loss and user-specified loss). }\end{array}$ \\
\hline 54 & $\begin{array}{l}\text { This option changes the two-phase to single phase vapor/gas transition truncation } \\
\text { limit in subroutine EQFINL for the semi-implicit scheme. }\end{array}$ \\
\hline 55 & $\begin{array}{l}\text { This option is a collection of modeling improvements designed to minimize } \\
\text { numerical sources of oscillations for low pressure two-phase flow simulations. } \\
\text { Specifically, this option affects: } \\
\bullet \quad \text { Interfacial heat transfer for annular mist } \\
\text { - Mist pre-CHF }\end{array}$ \\
$\begin{array}{l}\text { The liquid-side interfacial heat transfer coefficient has been modified to replace } \\
\text { "ad hoc" correlations with more physical models. }\end{array}$ \\
\hline
\end{tabular}




\subsubsection{Two volume insurge "Box" problem ${ }^{[5]}$}

The problem gauges the code's response during a simple fill transient in various boxes in the thermo-dynamic grid. Each of the many cases run concentrates on the worst boxes in the various regions of the grid. The two-volume input model is illustrated in Fig. 5.2.1.1. Single volume 503 has a geometry representative of the pressurizer of a PWR. Time-dependent junction 502 forces constant-rate flow into volume 503 from time-dependent volume 501 that applies constant pressure and temperature boundary conditions.

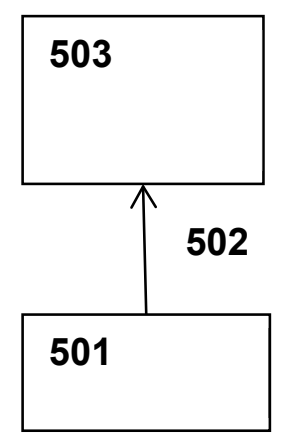

\section{Figure 5.2.1.1 Nodalization Diagram}

In the Box problems, the pressure and temperature in the model were set at the lowest values that still remained in the box of interest for the original steam tables (i.e. the lower left corner of the box). The flow rate was adjusted so that either the pressure or temperature reached the maximum value for the box at about $10 \mathrm{~s}$, at which time the calculation was terminated. Thus, the pressure and temperature of the single volume remained in the original box throughout the calculation.

The measure for code performance is the mass error. The analyses concentrated on the worst boxes in the various regions of the original thermodynamic grid.

Descriptions of the conditions in the various boxes are summarized in Table 5.2.1.1. The table shows the box number and the pressure and temperature at the midpoint of the box.

Table 5.2.1.1. Normalized mass errors during simple filling calculations

\begin{tabular}{|r|c|c|l|}
\hline Box & \multicolumn{2}{|c|}{ Midpoint values } & Comments \\
\hline & Pressure (MPa) & Temp. (K) & \\
\hline 113 & $1.10 \mathrm{E}-03$ & 275.33 & Liquid (worst box in Region 7) \\
\hline 341 & $1.50 \mathrm{E}-03$ & 286.25 & Vapor (worst box in Region 8) \\
\hline 1936 & $9.00 \mathrm{E}-02$ & 410.0 & Vapor (worst box in Region 10) \\
\hline 5629 & 7.50 & 377.5 & Liquid (average box in Region 1) \\
\hline 5651 & 7.50 & 617.50 & Vapor (average box in Region 2) \\
\hline 7400 & 16.00 & 293.75 & Liquid (worst box in Region 9) \\
\hline 7445 & 16.00 & 627.50 & Vapor (worst box in Region 2) \\
\hline 7446 & 16.00 & 632.50 & Vapor (worst box in Region 4) \\
\hline 7495 & 16.00 & 936.575 & Vapor (high temperature) \\
\hline 7556 & 17.25 & 622.5 & Liquid (worst box in Region 1) \\
\hline 7669 & 18.25 & 627.5 & Liquid (worst box in Region 3) \\
\hline
\end{tabular}

${ }^{1}$ Worst box based on normalized error in specific volume rather than the coefficient of thermal expansion. 
Fig. 5.2.1.2 shows that $\omega=0.0,0.5,1.0$ and -1.0 produce almost zero mass error and that the only one with significant mass error is $\omega=-2.0$. However poor the option $\omega=-2.0$ performs here and elsewhere, it helps reveal behavior in the other options in subsequent tests.

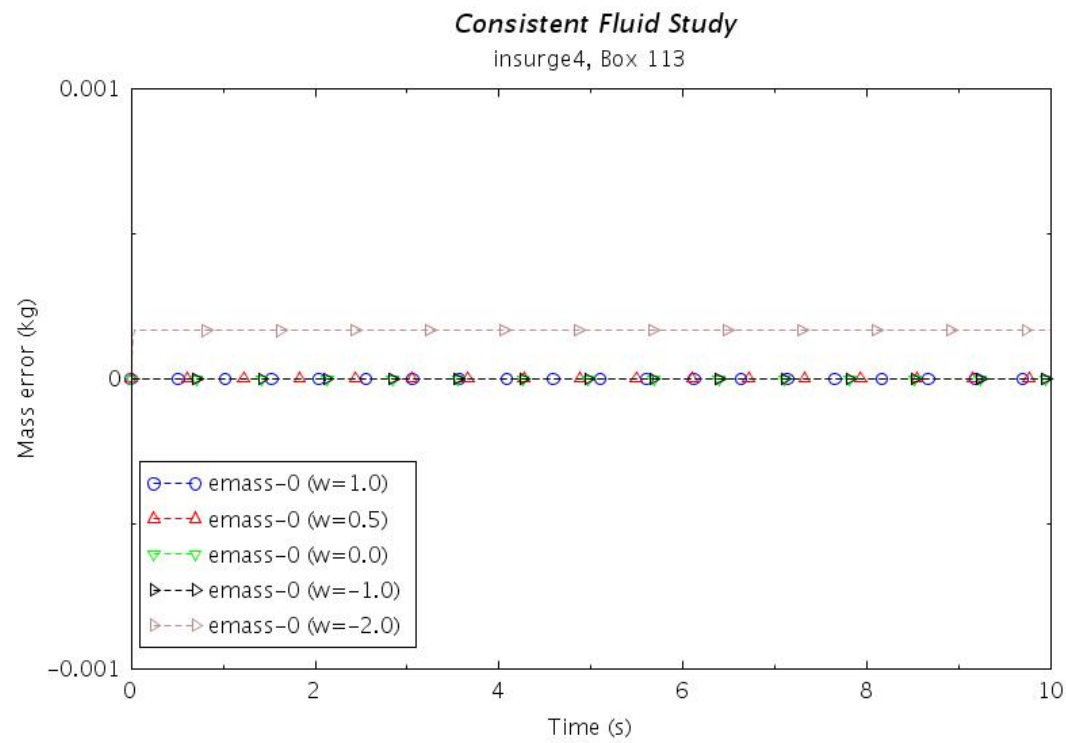

Figure 5.2.1.2 Mass Error for Box 113, all $\omega$ excellent, except $\omega=\mathbf{- 2}$.

In Fig. 5.2.1.3, $\omega(-2)$ is plotted as the upper curve. As $P$ approaches grid points, $\omega$ rises to 1.0 and the magnitude of the mass error increases. Otherwise, $\omega=-2$ tracks closely with $\omega=-1$. Except $\omega=0$, all plots have local extrema between P grid points. In Figs. 5.2.1.3a and 5.2.1.3b, local maxima in mass error and relative enthalpy error occur at the same times. In Figs. 5.2.1.3 and 5.2.1.4, the mass error of $\omega=-2$ closely parallels $\omega=-1$ except at pressure grid points and Mass $\operatorname{Error}(\omega=-2)-$ Mass $\operatorname{Error}(\omega=1)$ at the grid points are nearly constant.

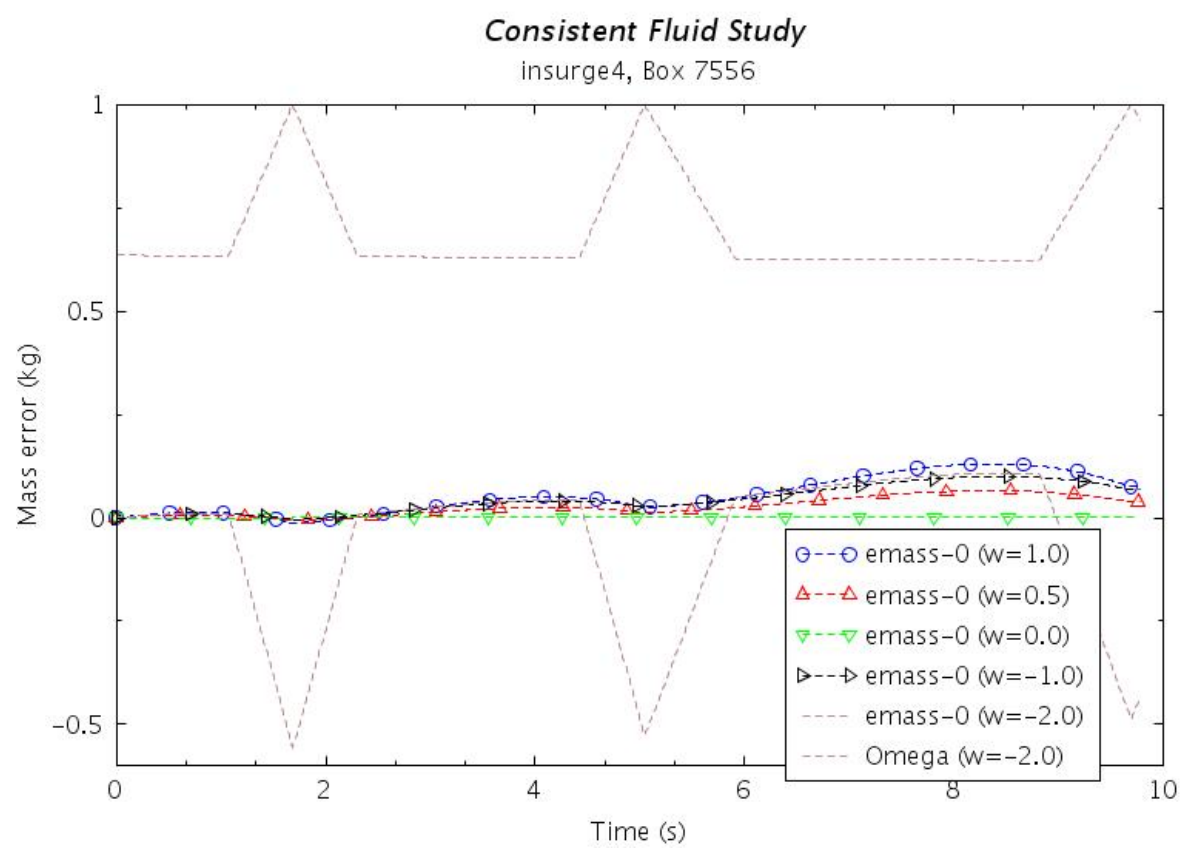

Figure 5.2.1.3a Box 7556 Mass error, $-1 \leq \omega<1$ outperform default RELAP5-3D 


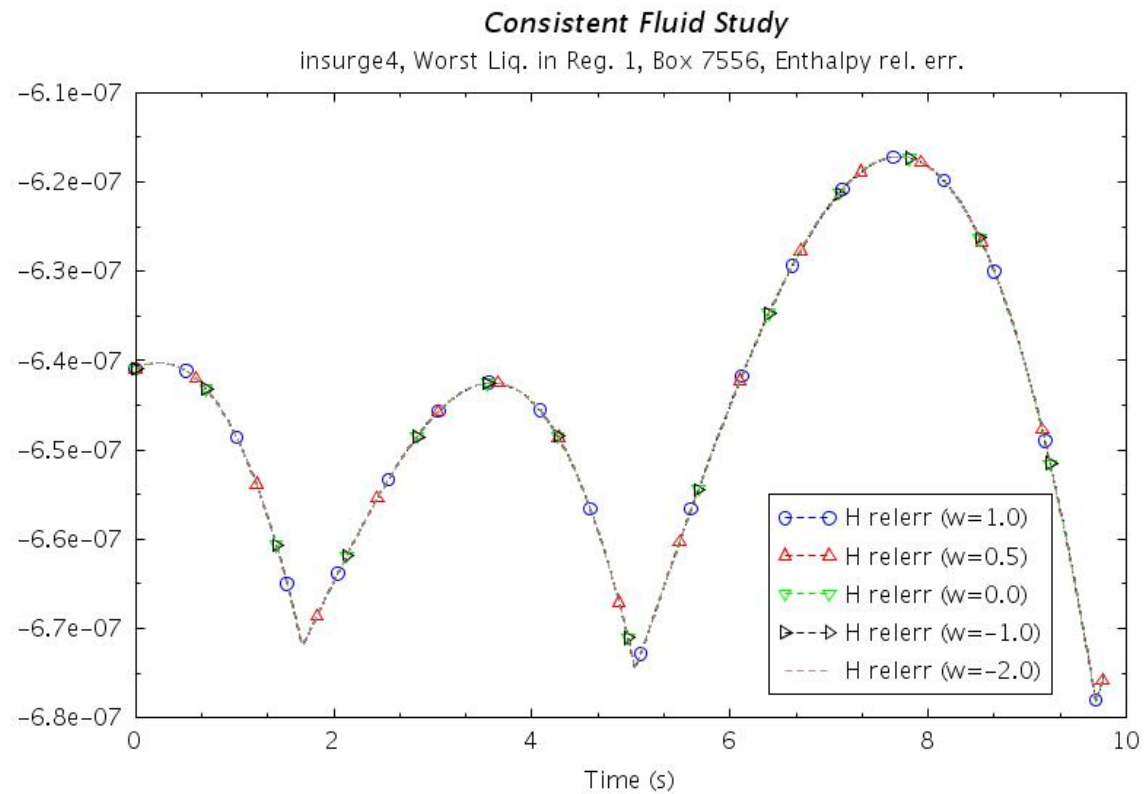

Figure 5.2.1.3b Box 7556 Enthapy error, $-1 \leq \omega<1$ outperform default RELAP5-3D

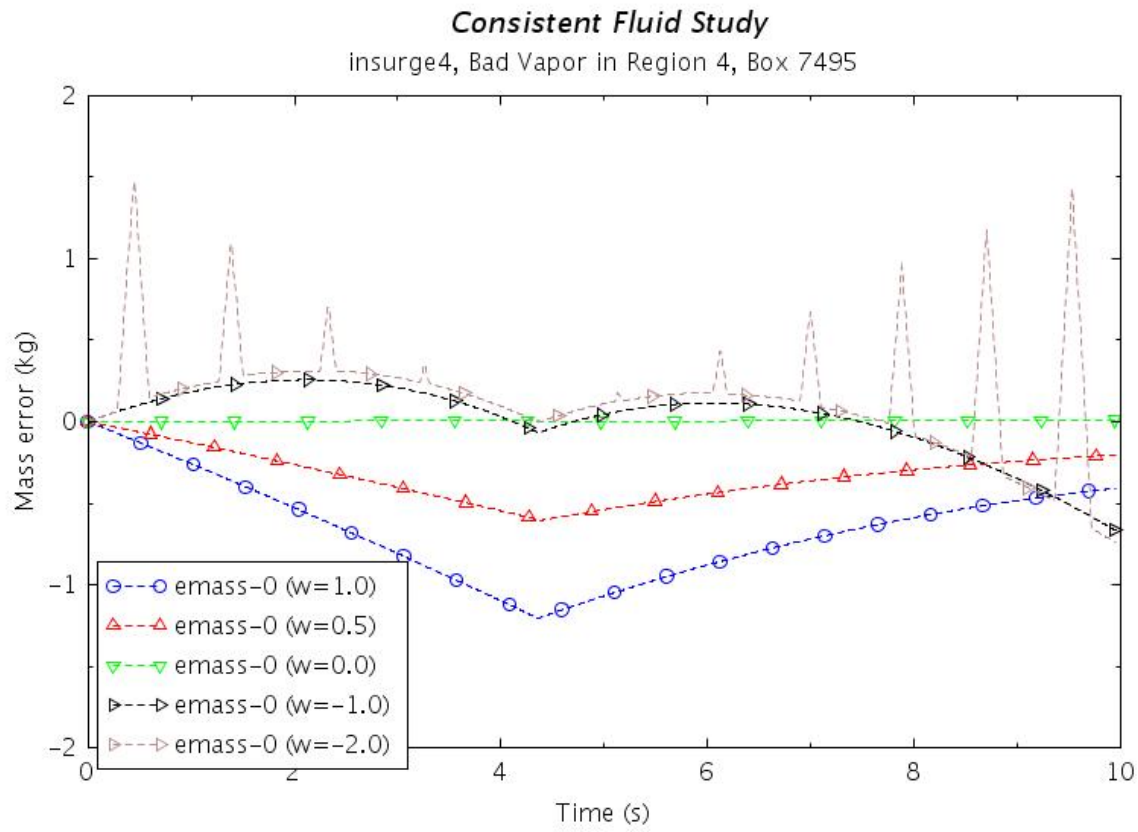

Figure 5.2.1.4 Box 7495 Mass error is smaller for $\omega=0.5$, nearly zero for $\omega=0.0$.

Fig. 5.2.1.5 shows the relationship between the spikes in mass error and pressure crossing the pressure grid lines. The pressure is modified to fit the upper half of the graph by the APTPLOT formula given in Eqn. (5.2-1) 


$$
\text { s5.y }=(s 5 . y * 1.0-15000000.0) / 2.1 \mathrm{e} 8
$$

Here s5.y is the APTPLOT reference to pressure. To produce the horizontal grid value lines on Fig. 5.2.1.5, the same transformation, namely Eqn. (5.2-1), is applied the relevant pressure values from the $\mathrm{H} 2 \mathrm{ON}$ fluid properties table. The pressure values are listed in the legend, namely, $\mathrm{P}=15088700.0,15276300.0,15463800.0,15651300.0,15838800.0,16026200.0$, $16401300.0,16580600.0,16747200.0$, and 16915500.0.

Note that mass error in all graphs except $\omega=0.0$ undulate with local minima in mass error at the pressure grid points.

\section{Consistent Fluid Study}

insurge4, Worst Liquid in Region 9, Box 7400

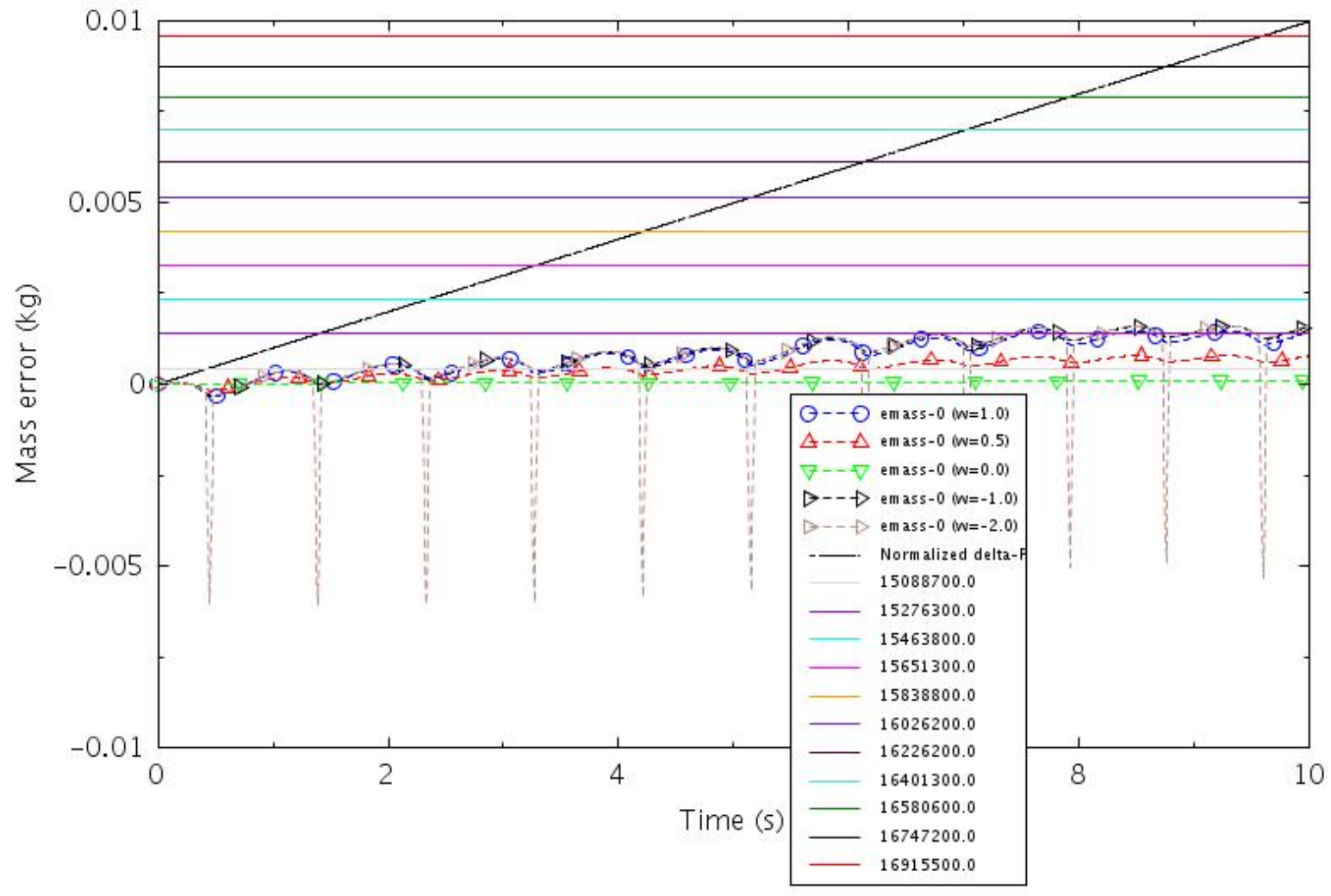

Figure 5.2.1.5 Box 7400 Mass error has local minima at pressure grid values. 
In Fig. 5.2.1.6a, all $\omega$ graphs have local minima at the pressure grid point, except $\omega=0$ with $\omega=-2.0$ having the largest mass error. Fig. 5.2.1.6b indicates that all $\omega \neq 0$ have local maxima midway between pressure grid points.

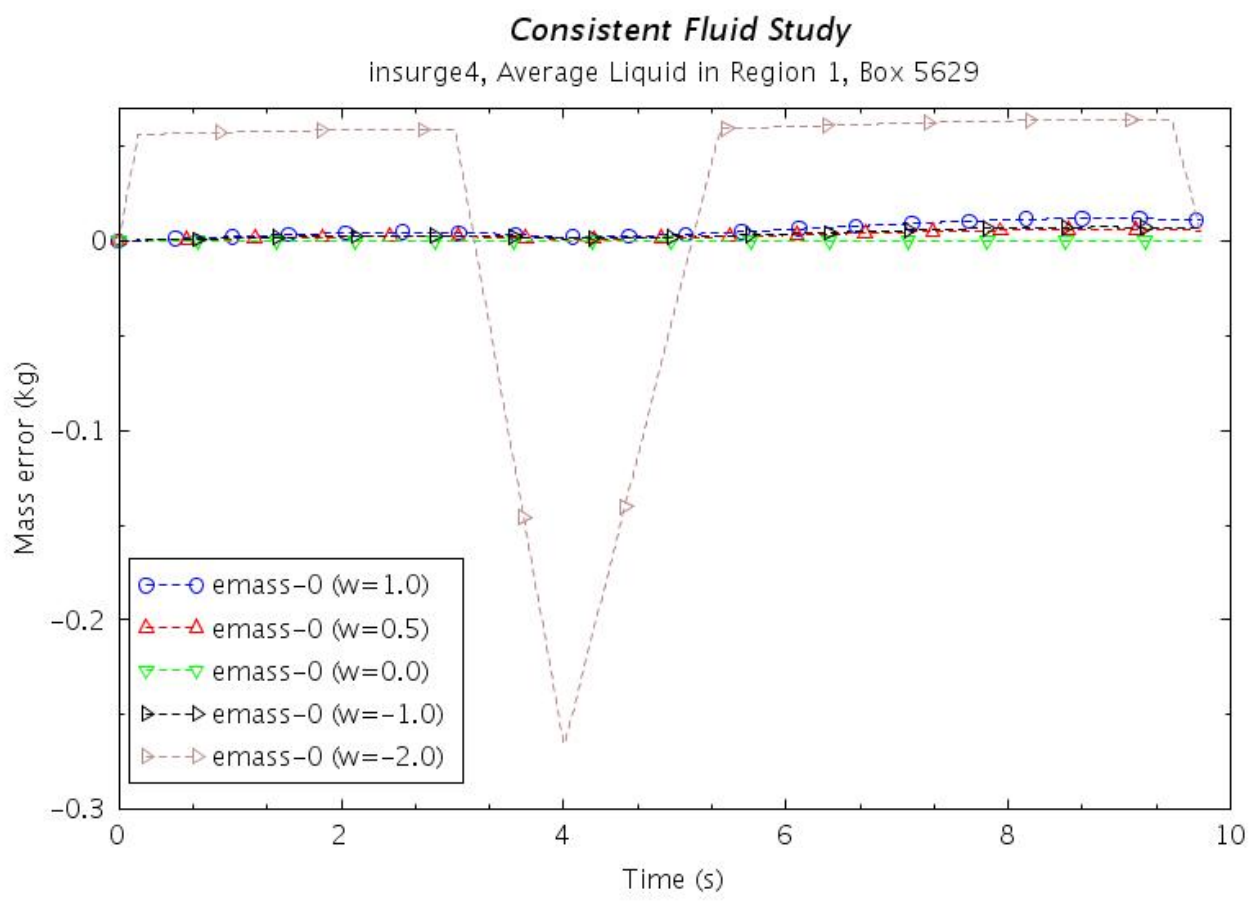

Figure 5.2.1.6a Mass Error for Box 5629, $\omega=0$ excellent, $\omega=-2$ spikes.

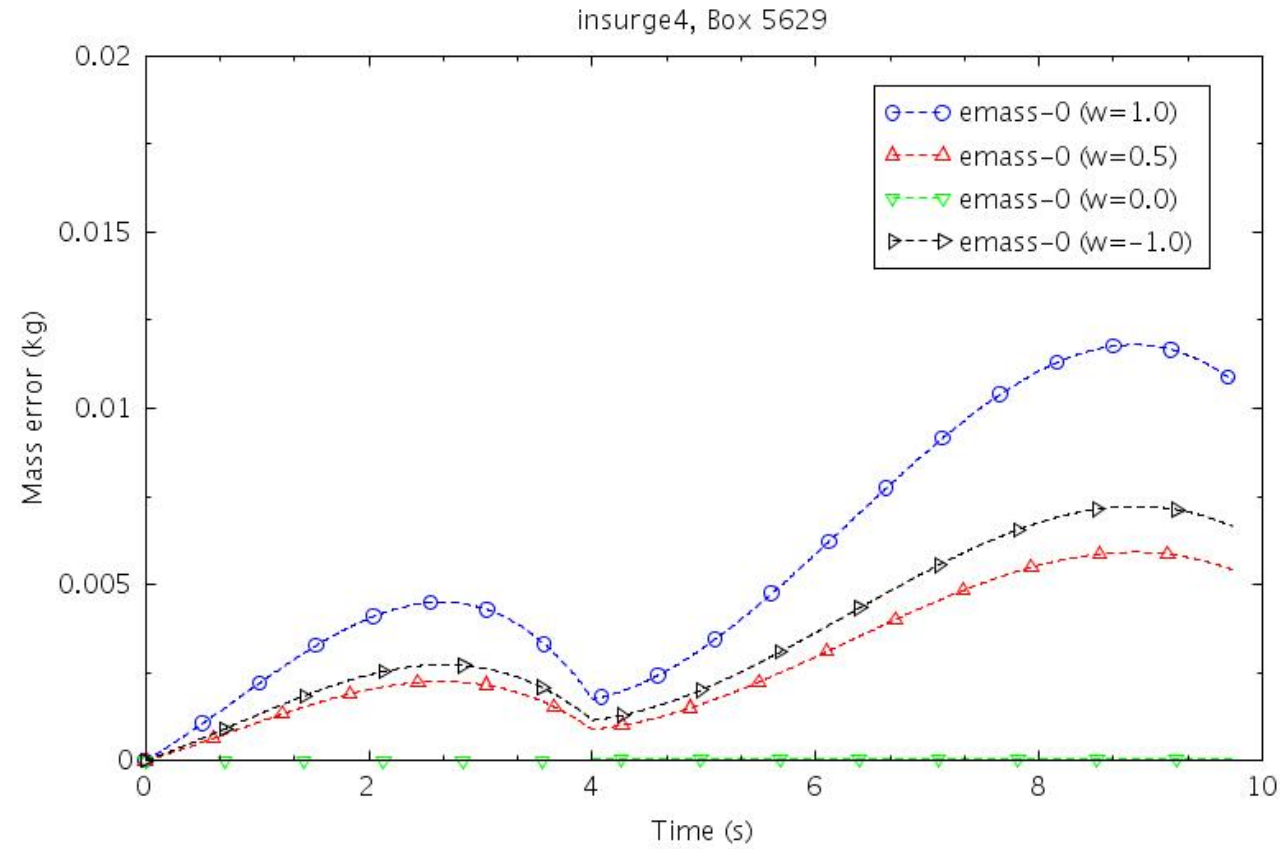

Figure 5.2.1.6b Zoom for Box 5629, $\omega \neq-2$ outperform default RELAP5-3D 
In Figs. 5.2.1.7, 5.2.1.8, 5.2.1.9a and 5.2.1.9b, values of $\omega<1$ generally produce lower mass error than default RELAP5-3D. The graph for $\omega=-2$ closely mirrors that of $\omega=-1$, except at $P$ grid points. Figs. 5.2.1.7, 5.2.1.8 have no undulation at $P$ grid points. Box 7445 and 7446 cross no u grid point.

Consistent Fluid Study

insurge4, Worst Liquid in Region 2, Box 7445

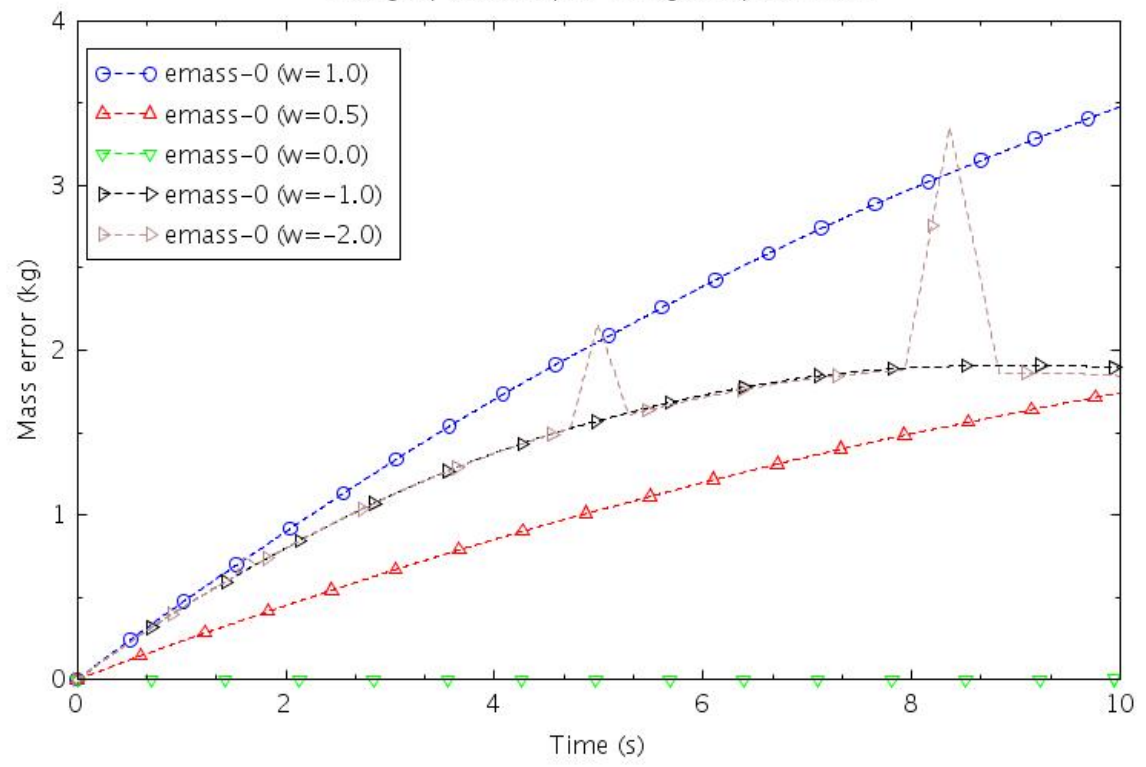

Figure 5.2.1.7 Mass error in Box 7445, all $\omega<1$ outperform default RELAP5-3D

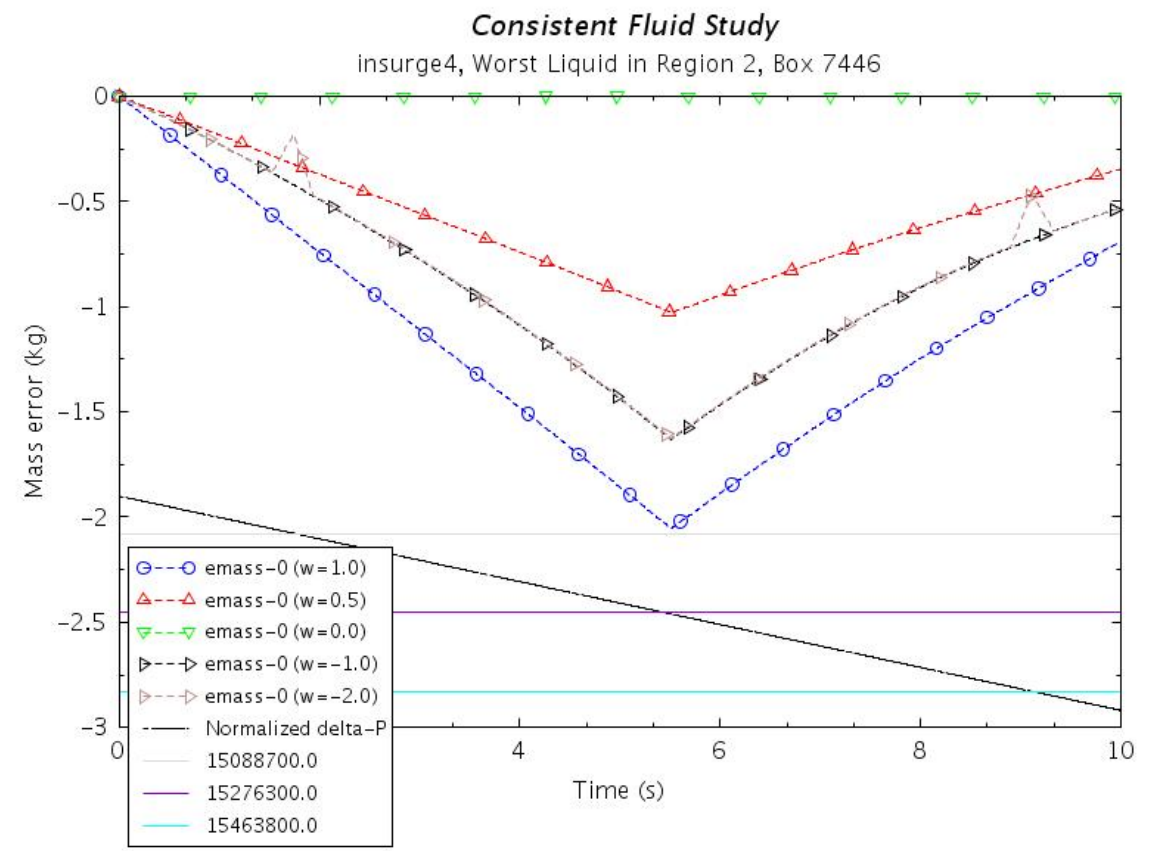

Figure 5.2.1.8 Box 7446 Mass error: all $\omega$ good. No u-crossing, corner at middle p-point. 
Box 1936 crosses a u grid point. Comparing Figs. 5.2.9a and 5.2.9b, $\omega=-2,-1,0.5,1$ have an inflection point at the $u$ grid point. Also, $\omega=-2$ has corners a pressure grid points, $\omega=-1$ has a max at $\omega(u)=0.5$.

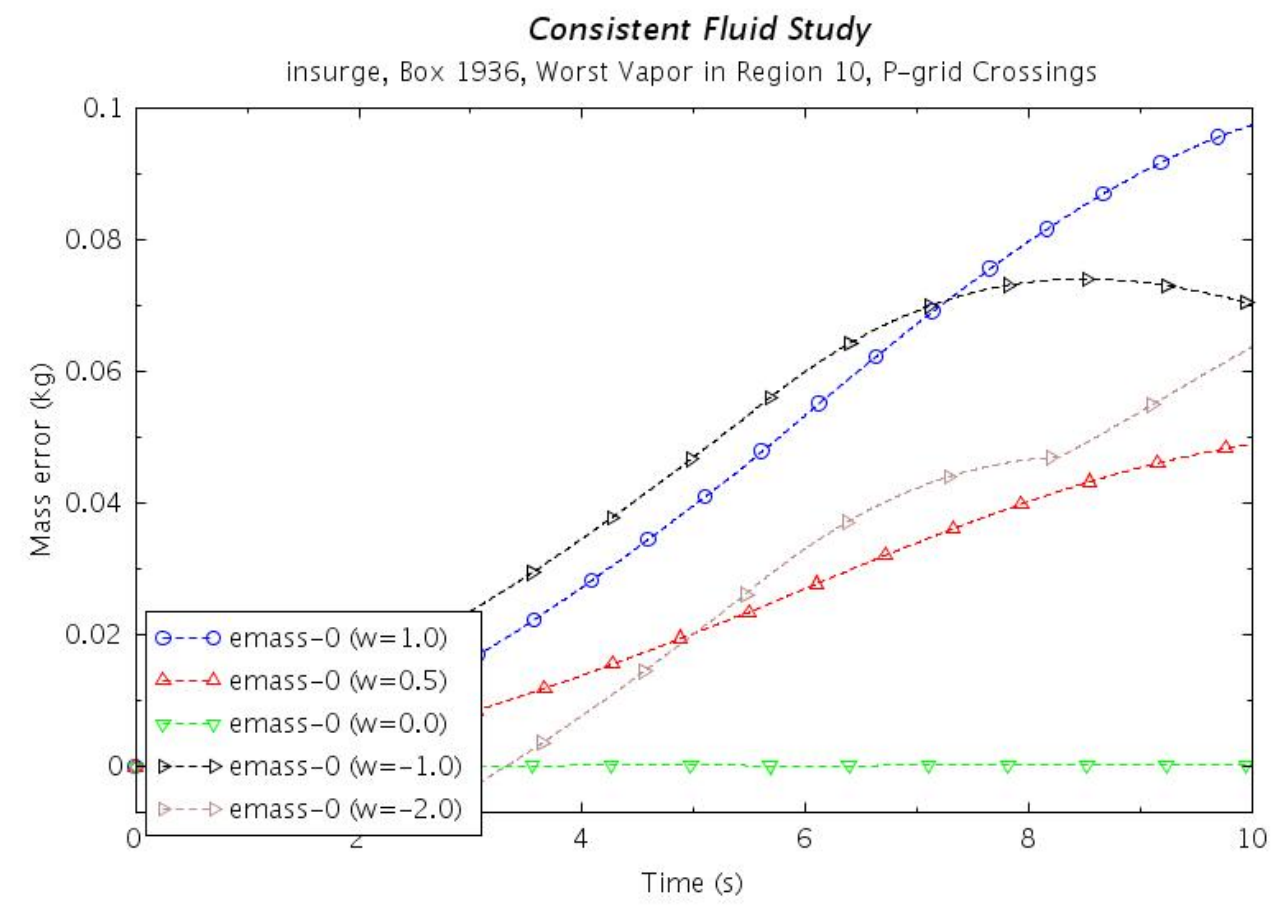

Figure 5.2.1.9a Mass Error for Box 1936, all $\omega \neq 1.0$ better than default, $\omega=0$ excellent.

\section{Consistent Fluid Study}

insurge Box 1936, emass uncorrelated to omega value, grid crossings

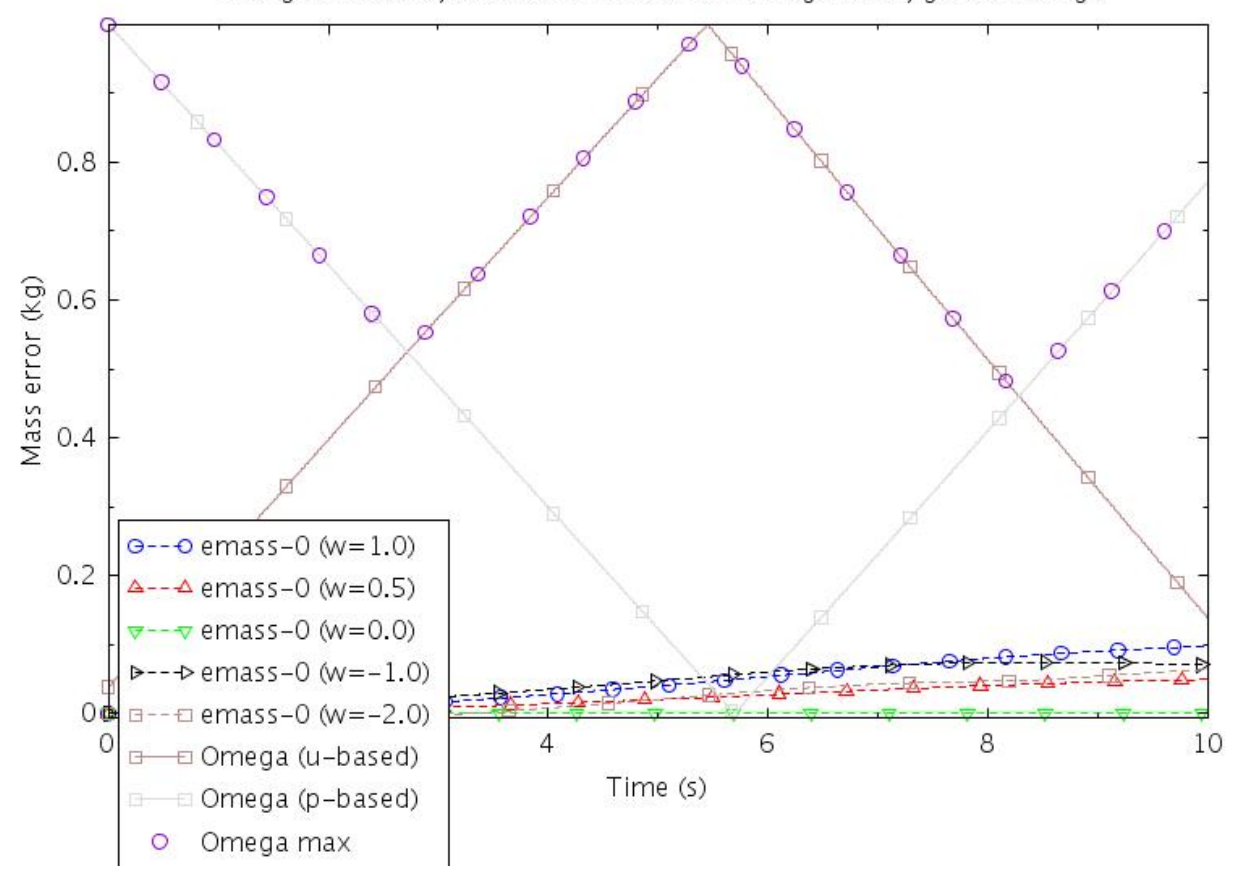

Figure 5.2.1.9b Mass Error for Box 1936, all $\omega \neq 1.0$ better than default, $\omega=0$ excellent. 
In Figs. 5.2.10 and 5.2.11, negative values of $\omega$ perform poorly, while increasing $\omega$ from 0.0 to 1.0 results in increasing mass error.

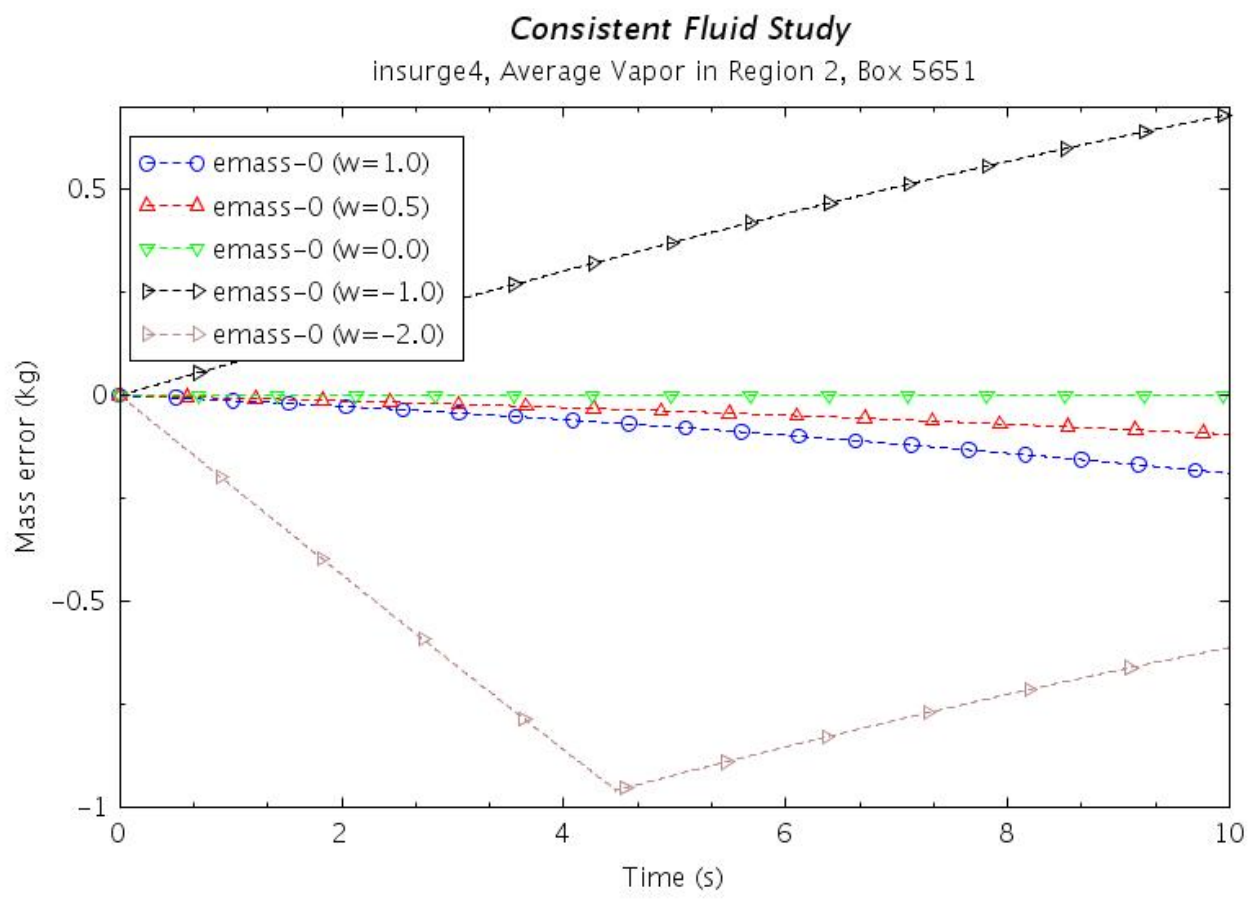

Figure 5.2.1.10 Mass Error for Box 5651, all $\omega=0.5,1.0$ good, $\omega=0$ good, $\omega<0$ poor.

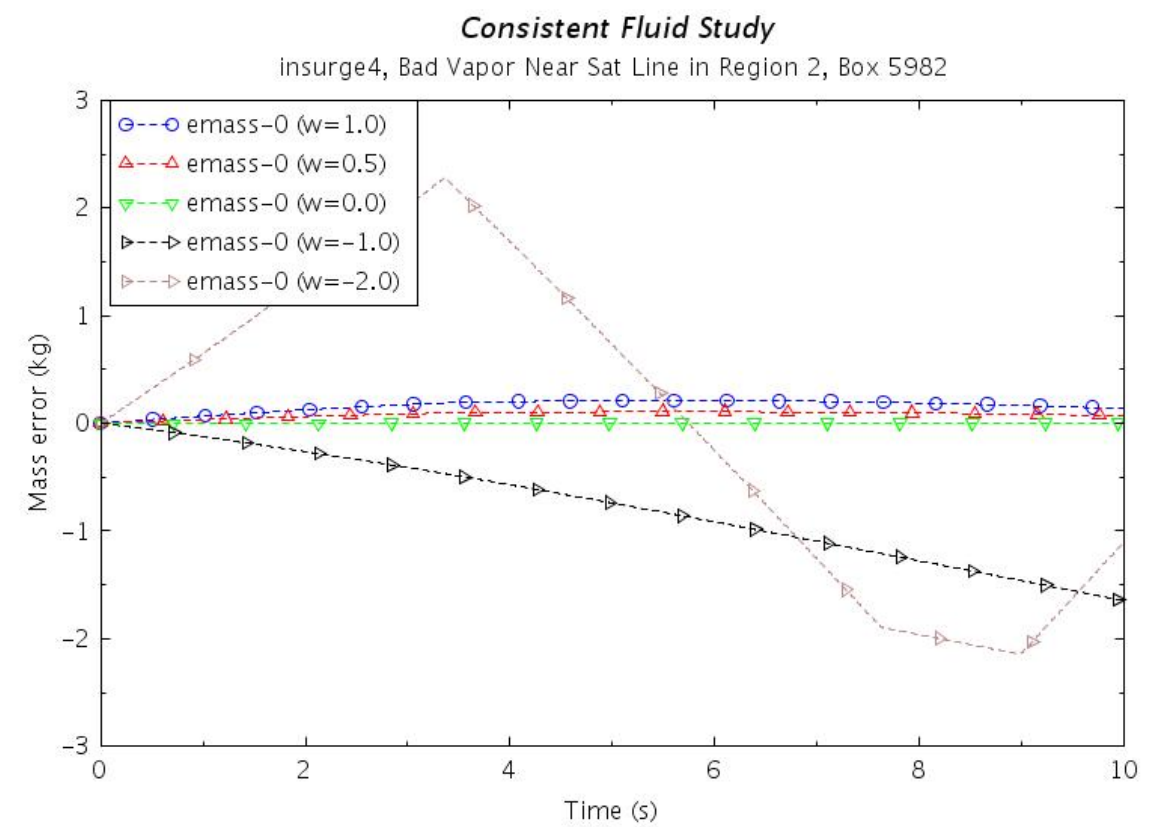

Figure 5.2.1.11 Mass error in Box 5928, $1>\omega \geq 0$ outperform default RELAP5-3D 
In Fig. 5.2.12 shows that all values of $\omega$, except -2, outperform default RELAP5-3D. Mass error increases as $\omega$ increases $\omega$ from 0.0 to 1.0. Except for $\omega=0$, the graphs undulate between $P$ grid points.

\section{Consistent Fluid Study}

insurge4, Worst Liquid in Region 3, Box 7669, P-grid Crossings

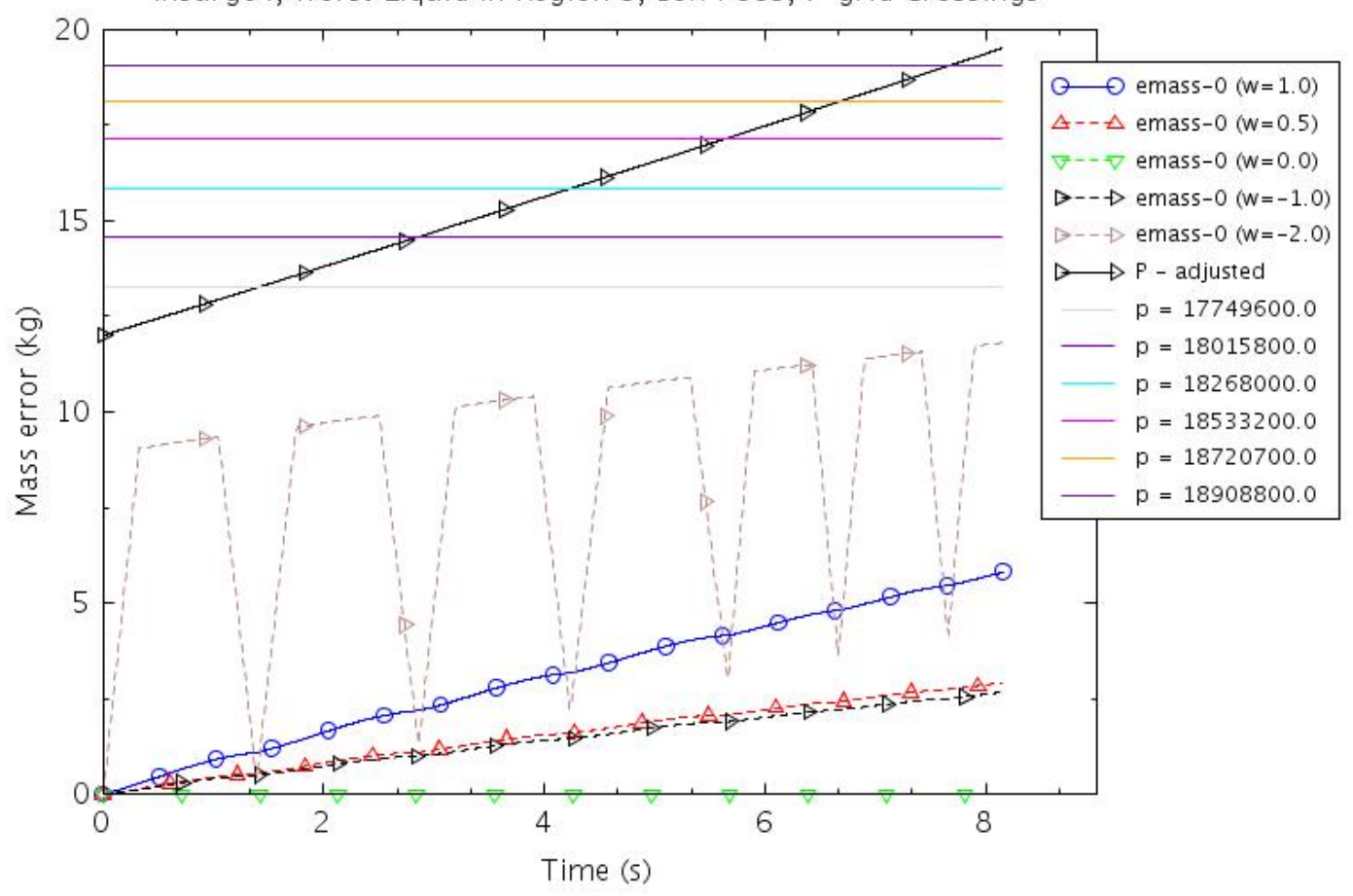

Figure 5.2.1.12 Mass error in Box 5928, $1>\omega \geq-1$ outperform default RELAP5-3D

The six cases numbered sc1 through sc6 show that $\omega=0$ produces almost no mass error, and that $\omega=0.5$ always has smaller mass error than default RELAP5-3D. Except in the final two cases, $\omega=-1$ also produces less mass error. Only in Case sc3 does $\omega=-2$ outperform the default code. Otherwise, it produces the most mass error while mirroring $\omega=-1$ except at pressure grid points. 
Consistent Fluid Study

insurge4, Liquid Near Critical Point, Box sc1

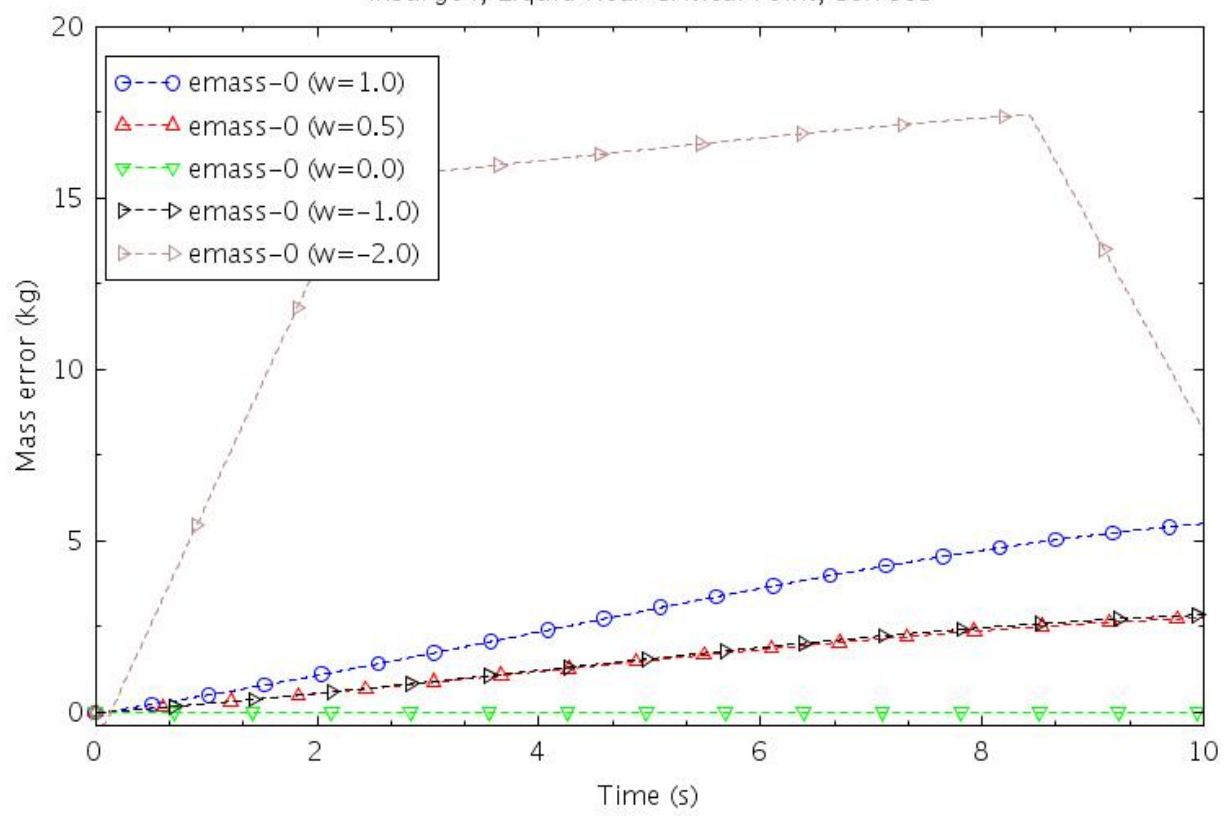

Figure 5.2.1.13 Box sc1 Mass error, $\omega \neq-2$ outperform default RELAP5-3D

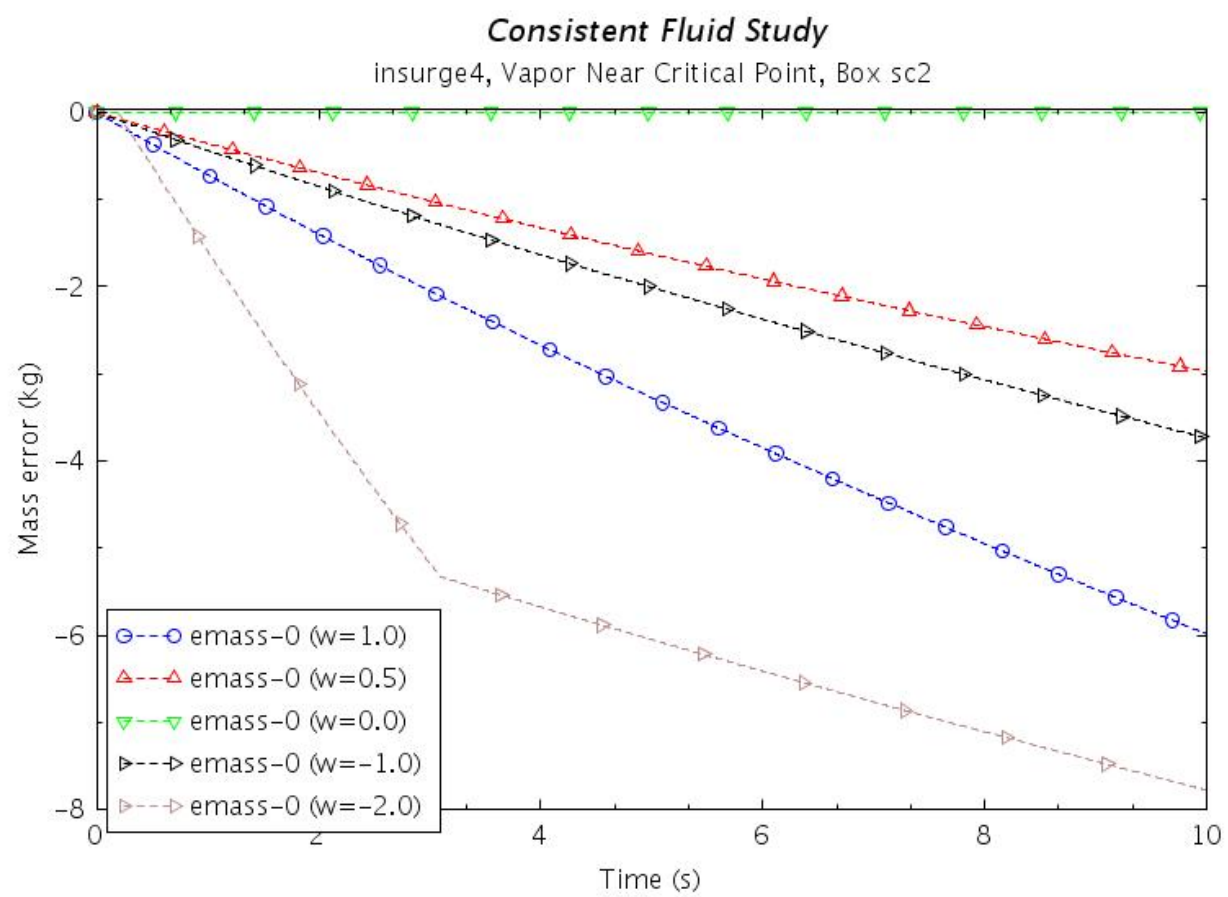

Figure 5.2.1.14 Box sc2 Mass error, $\omega \neq-2$ outperform default RELAP5-3D 


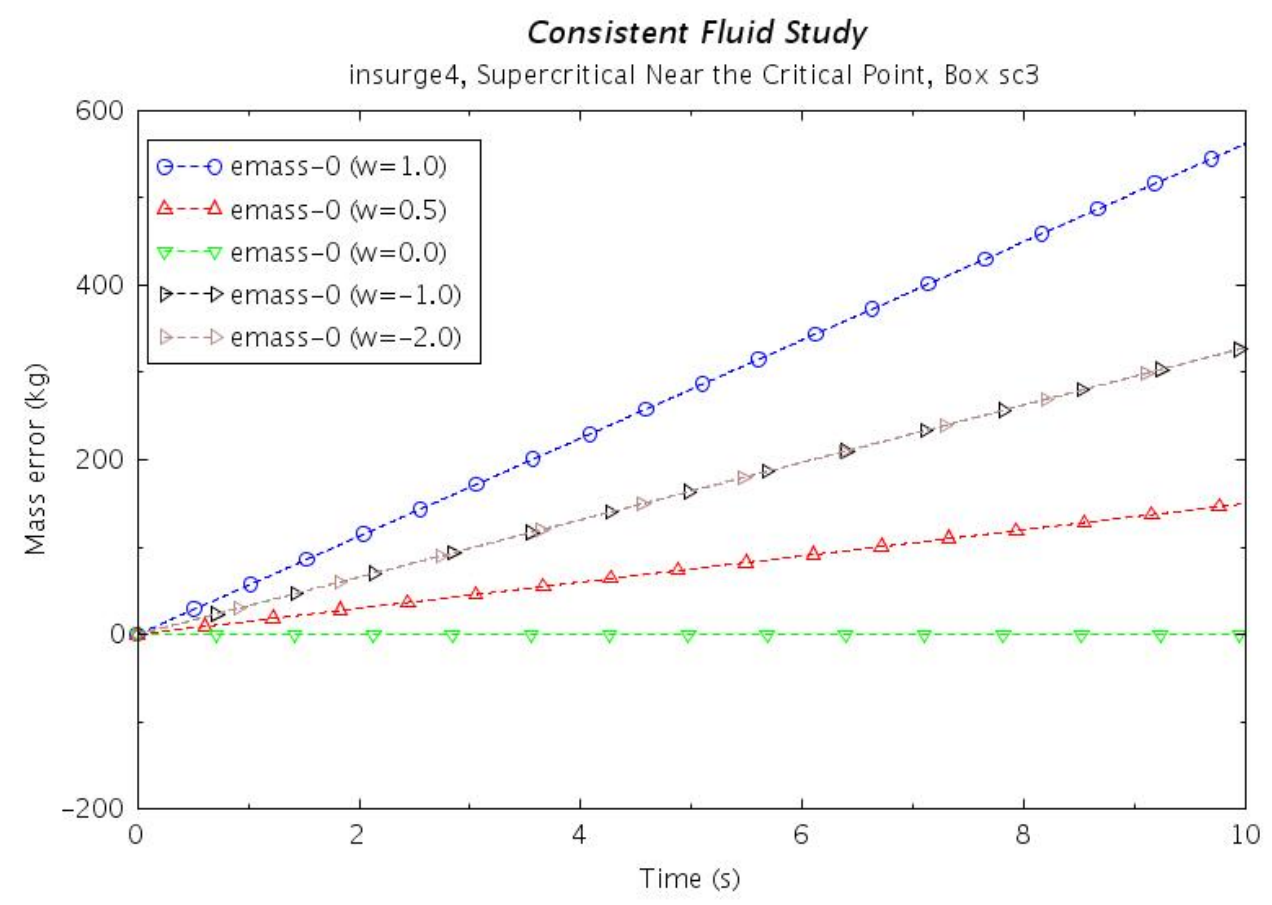

Figure 5.2.1.15 Box sc3 Mass error, all $\omega<1$ outperform default RELAP5-3D

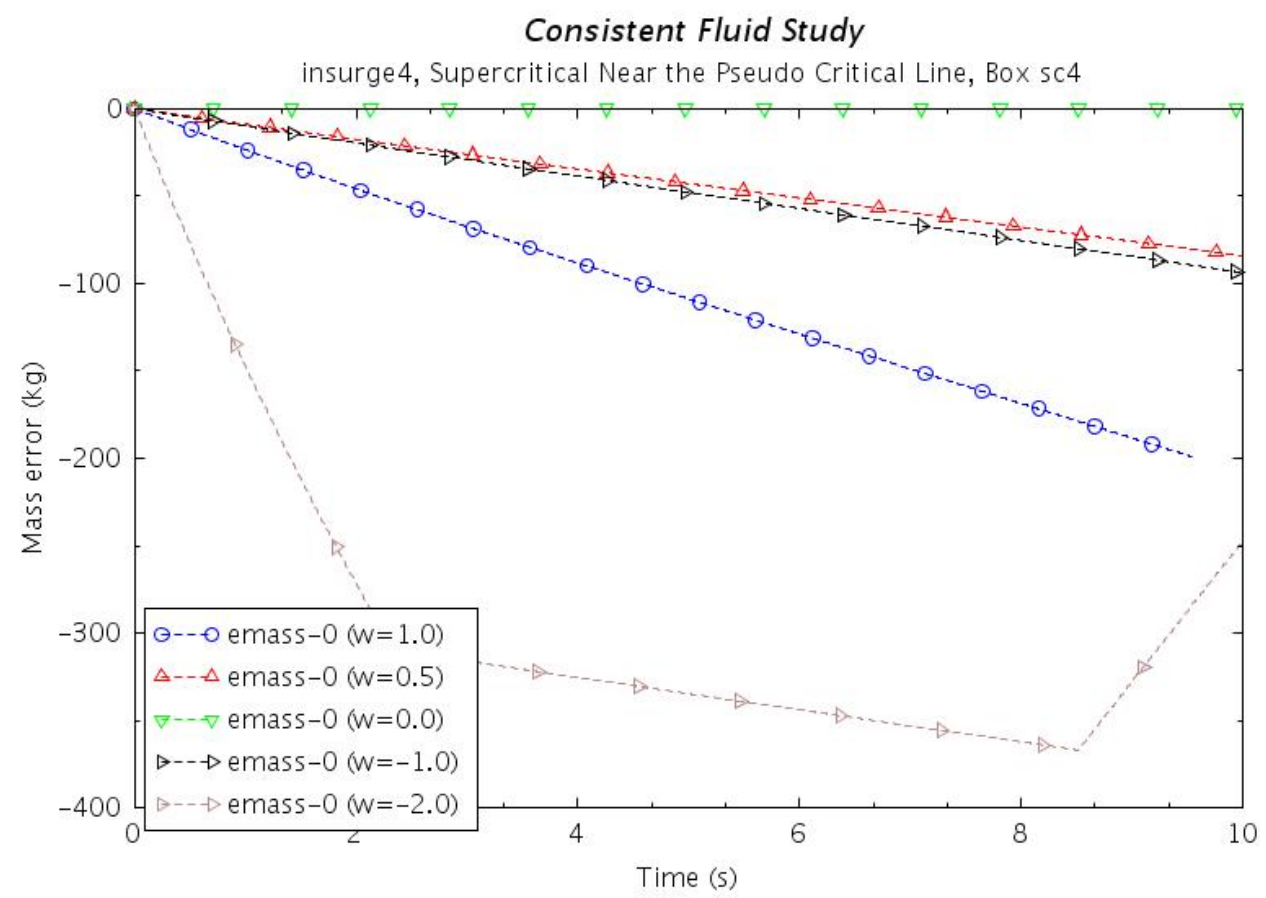

Figure 5.2.1.16 Box sc4 Mass error, $\omega=-2$ outperform default RELAP5-3D 


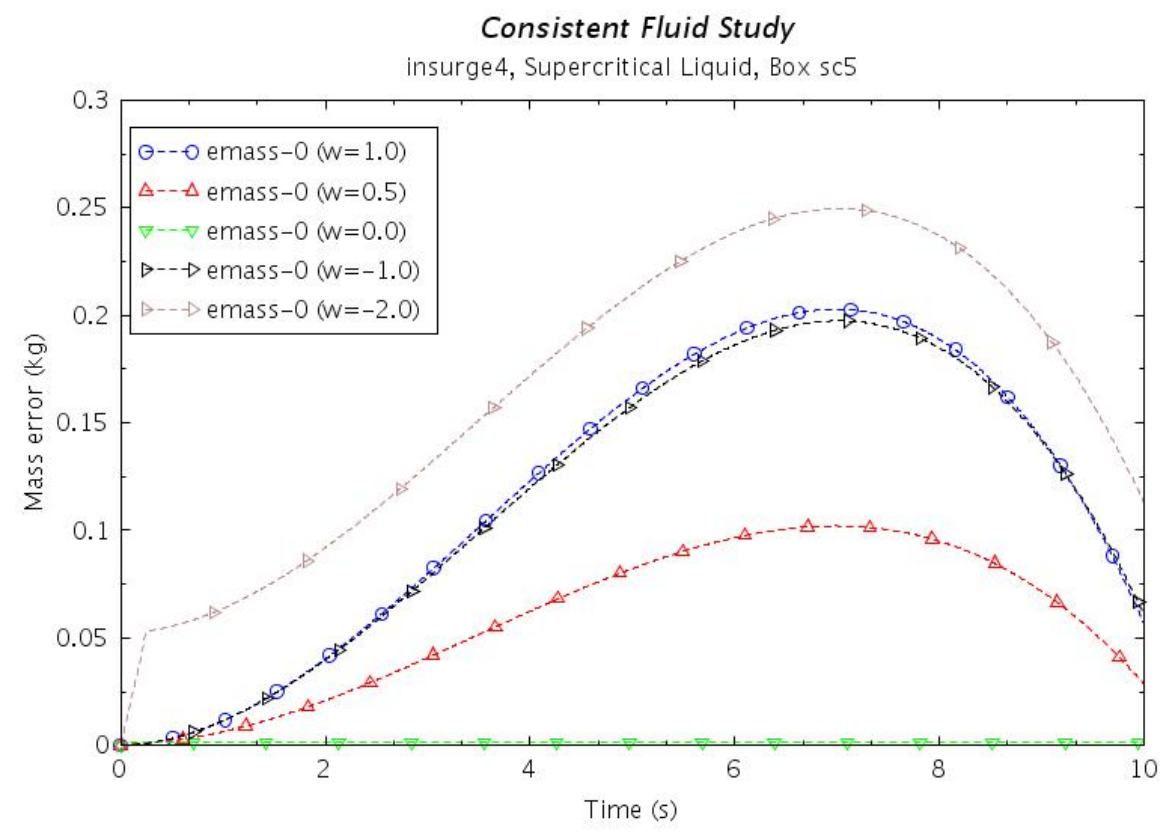

Figure 5.2.1.17 Box sc5 Mass error, $\omega \neq-2$ outperforms default RELAP5-3D

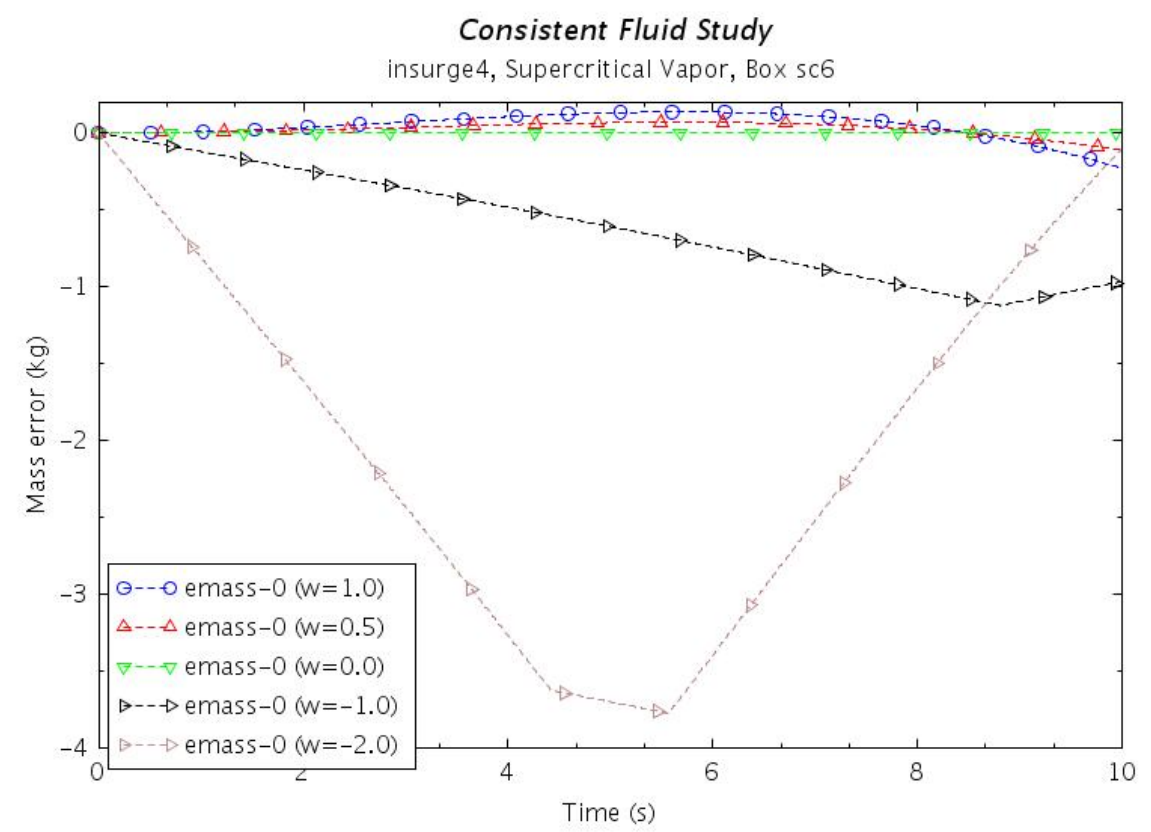

Figure 5.2.1.18 Box sc6 Mass error, $0 \leq \omega<1$ outperforms default RELAP5-3D

For the insurge problems, the option $\omega=0$ is clearly best. Both $\omega=0.5$ and $\omega=-1$ are generally better than the default $\omega=1$. At P-gridline crossings, mass error hits local maxima. No differences in mass error between INL and BTS card-1 options are visible. 


\subsubsection{Two volume outsurge "Box" problem}

The nodalization diagram of the insurge and outsurge problems are identical. The purpose of the outsurge problems is to put the pressurizer volume through a blowdown, causing a phase boundary crossing. Also, the flow has been reversed. Only two cases are considered.

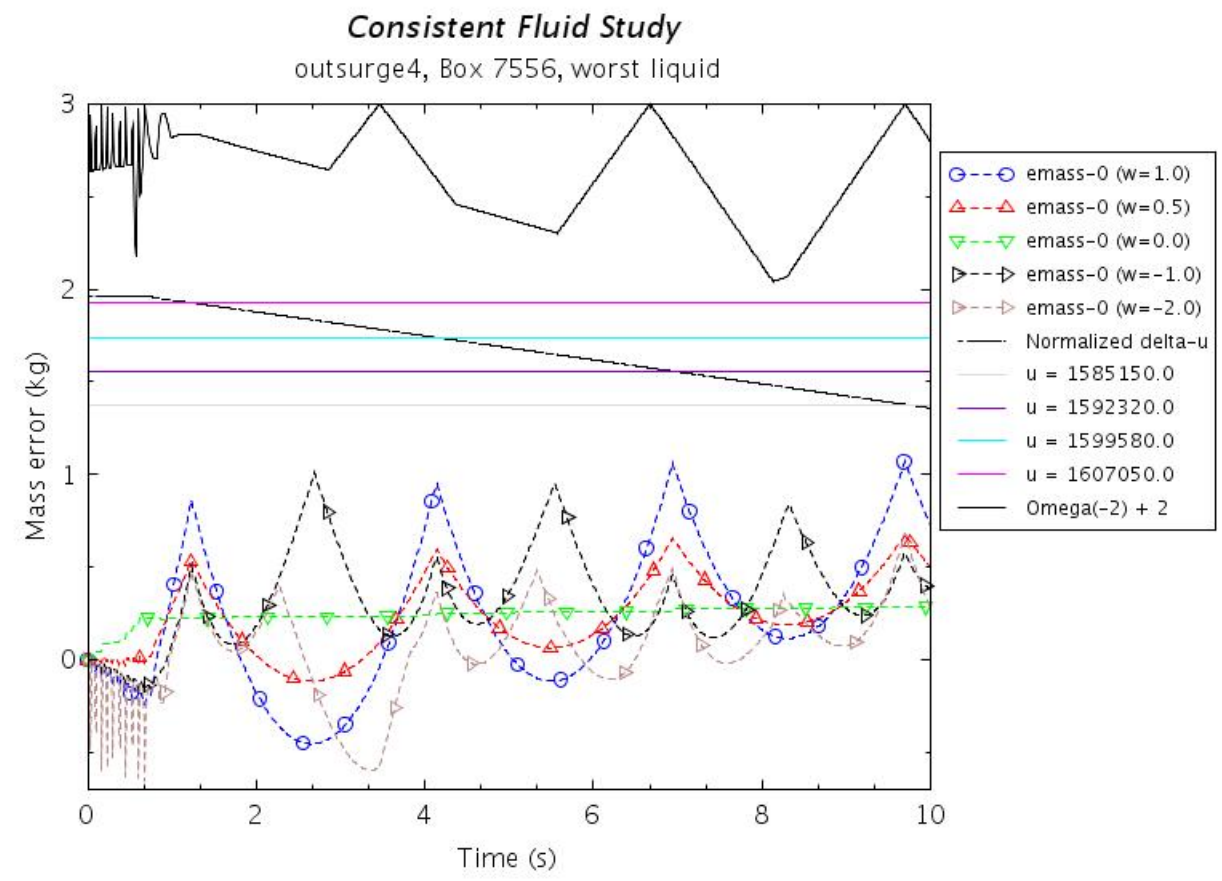

Figure 5.2.2.1a 7556: $\omega(-2)$, u-gridline crossings, and mass error for INL options

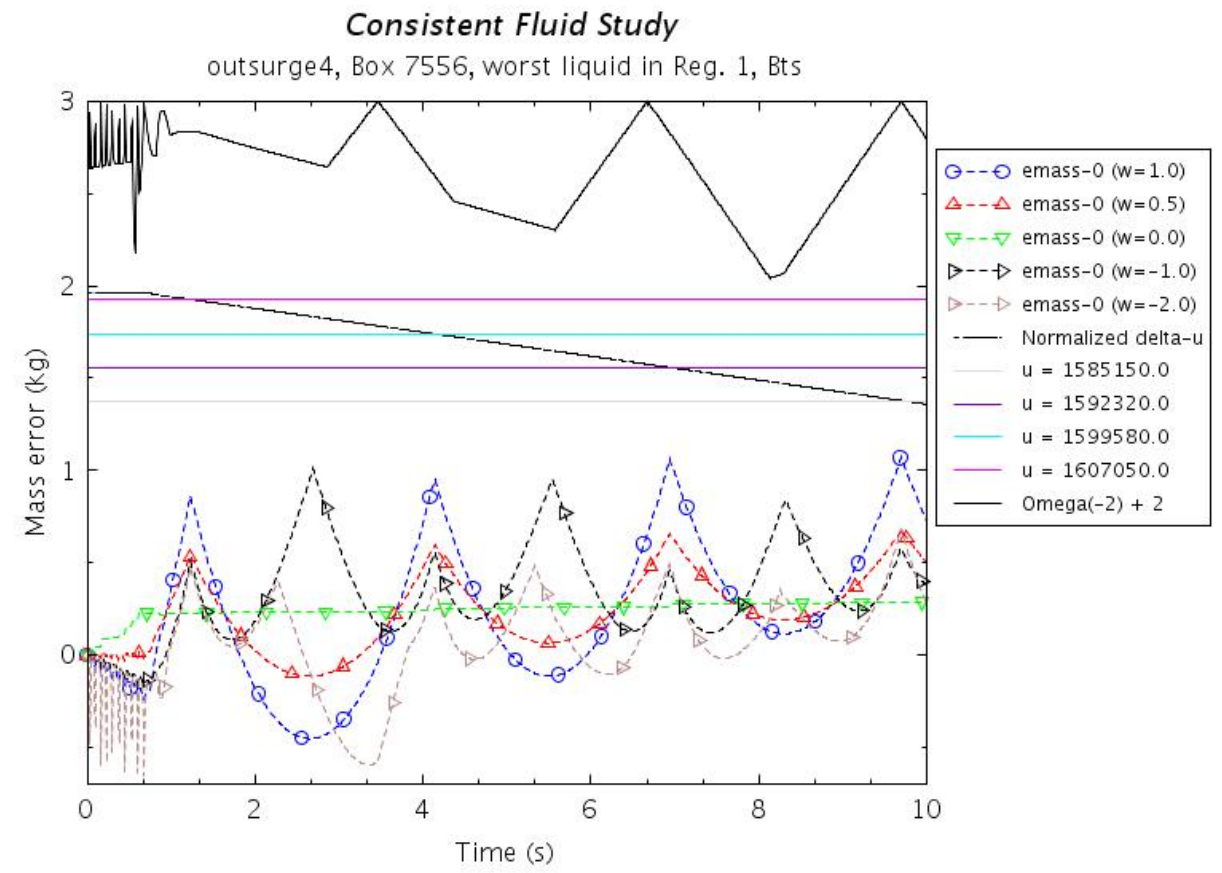

Figure 5.2.2.1b 7556: $\omega(-2)$, u-gridline crossings, and mass error for BTS options 


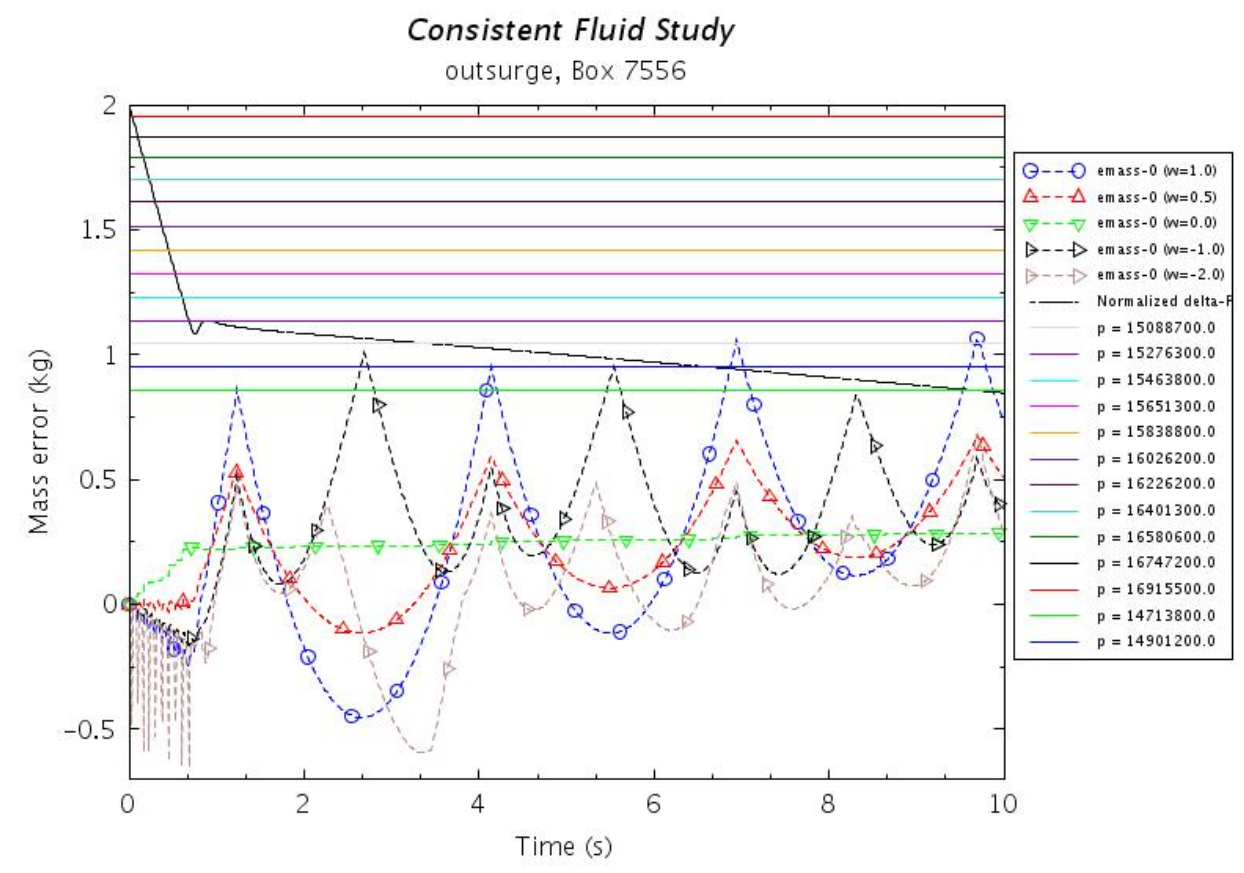

Figure 5.2.2.2a 7556: P-gridline crossings, and mass error for INL options

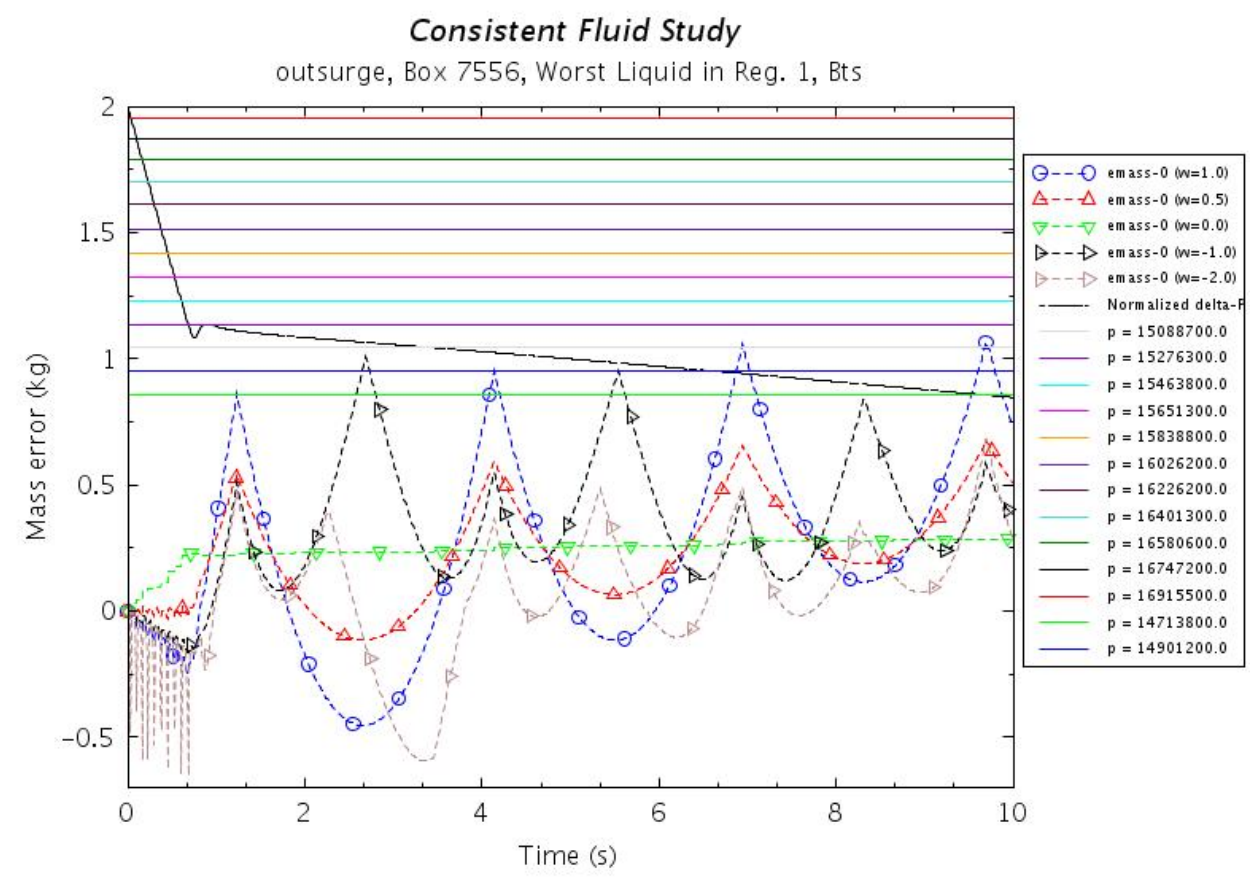

Figure 5.2.2.2b 7556: P-gridline crossings, and mass error for BTS options 


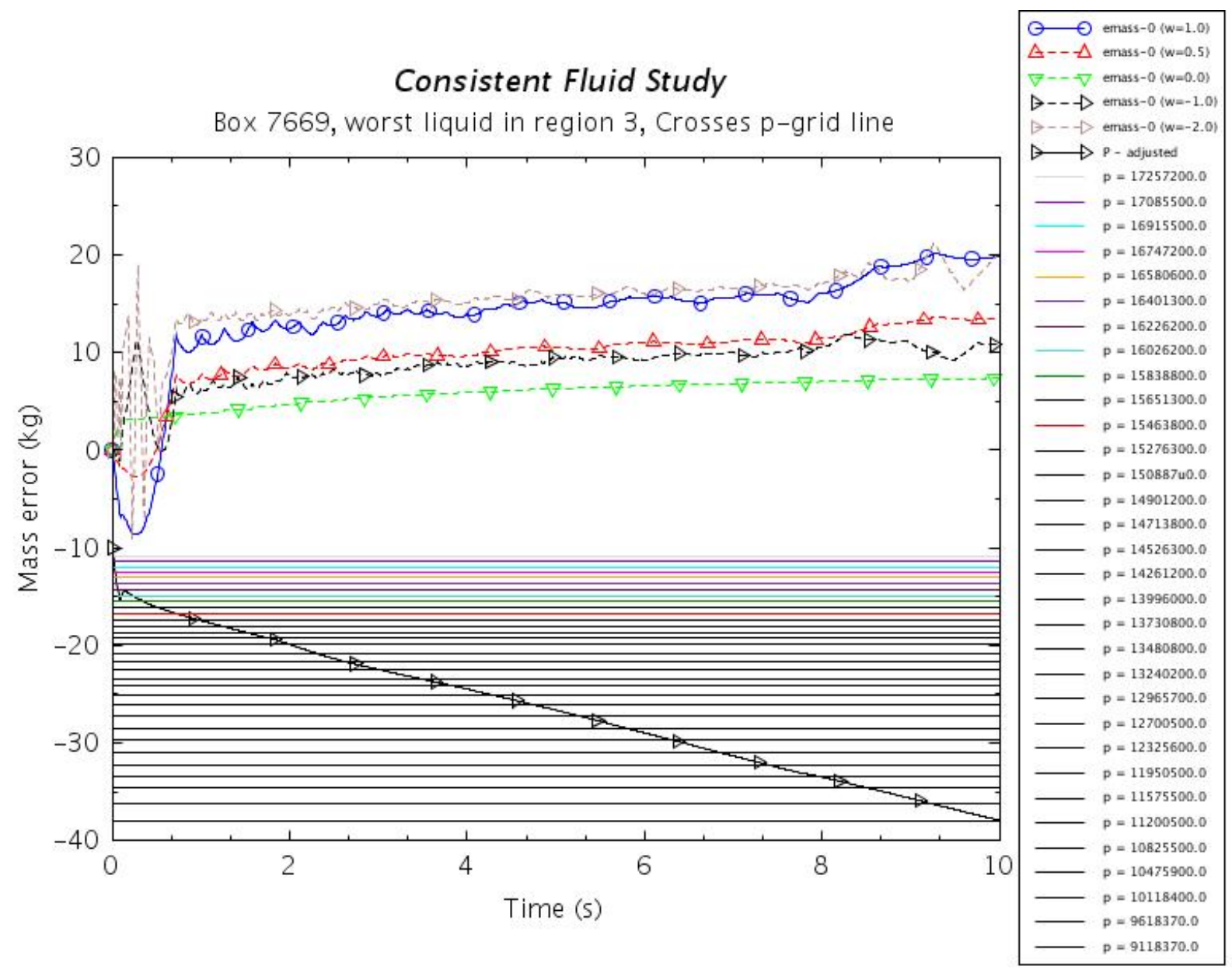

Figure 5.2.2.3b 7669: P-gridline crossings, and mass error for INL options 


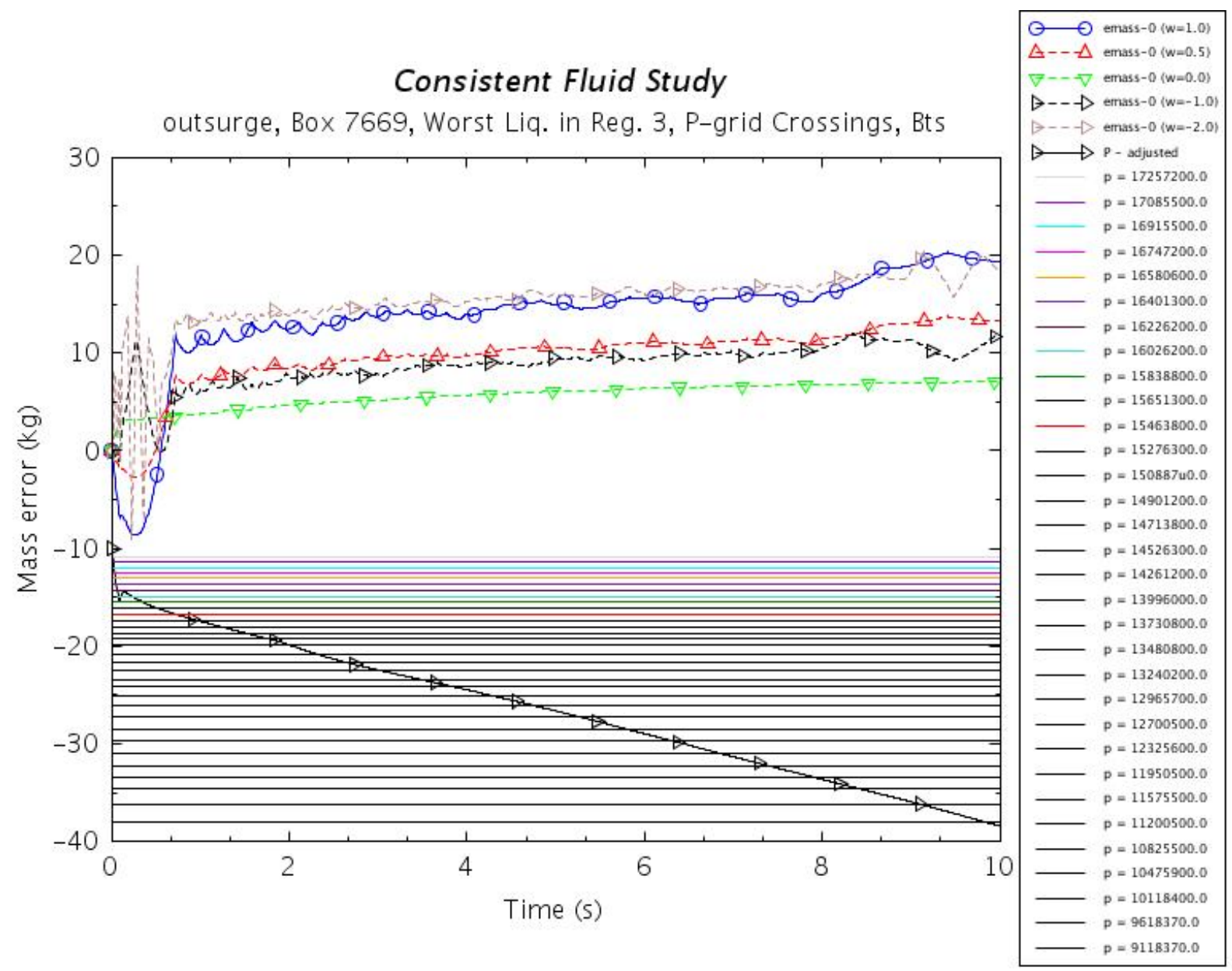

Figure 5.2.2.3b 7669: P-gridline crossings, and mass error for BTS options

There are no obvious differences in the mass error or pressure between the normal INL card-one options and the BTS options. This holds true for both Boxes 7769 and 7556 for any omega value. The following observations are made:

- As with insurge, the plot of $\omega(-2)$ exhibits jittery behavior and has local extrema at pressure gridline crossings.

- The plots of $\omega=1.0$ and $\omega=0.5$ have local maxima where the specific internal energy crosses gridlines.

- $\quad$ The smallest mass error is created when $=0.0$. However, unlike with insurge, the mass error is positive in outsurge problems.

- The second lowest mass error for Box 7556 comes when omega=-2.

- The second lowest mass error for Box 7669 comes when omega=-1. 


\subsubsection{Edwards Pipe}

The Committee on the Safety of Nuclear Installations (CNSI) identified the Edward's O'Brien Blowdown Test as Standard Problem number $1^{[6]}$ for performing validation of nuclear reactor safety computer codes. Fig. 5.2.1 shows the diagram of the Edward's O'Brien Blowdown Test facility ${ }^{[7]}$ conducted to investigate and measure pressurized two-phase water blowdown behavior in a straight pipe geometry. Fig. 5.2.2.1 gives the nodalization diagram for this classic separate effects code benchmark problem.

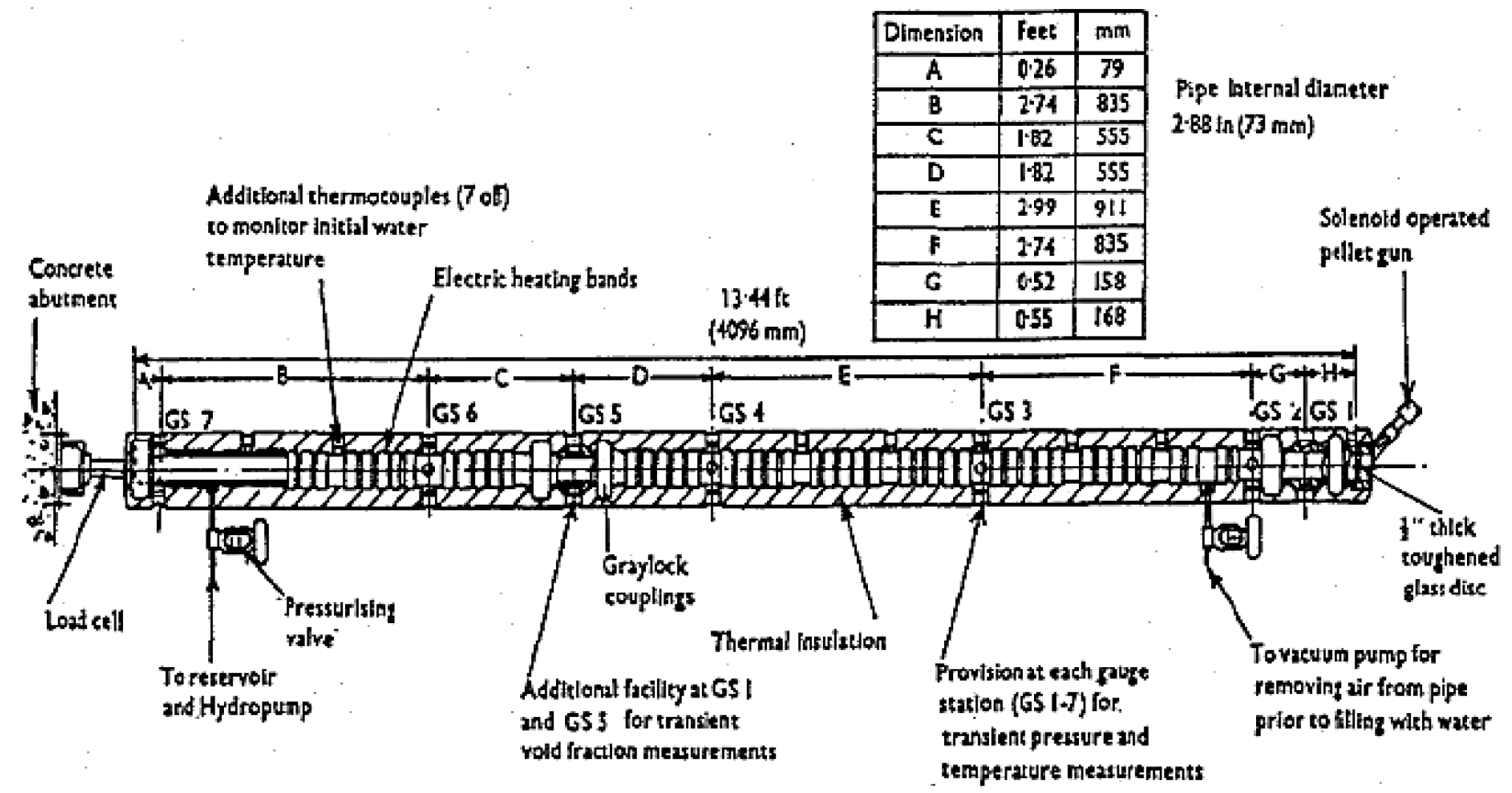

Figure 5.2.1.1 Diagram of the Edward's O'Brien Blowdown test facility
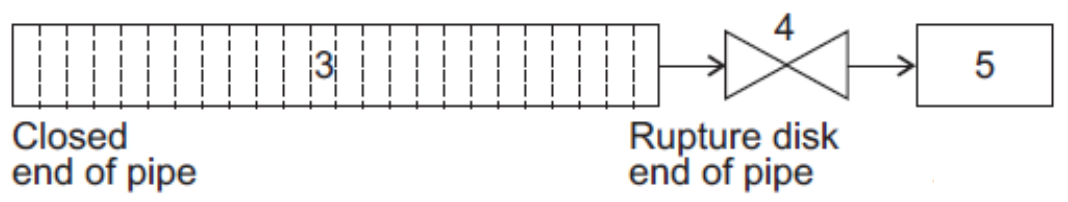

Figure 5.2.2.2 Diagram of the Edward's O'Brien Blowdown test facility

Again, $\boldsymbol{\omega}=\mathbf{0 . 0}$ produces the smallest mass error. However, unlike with the Box problems in Sec. 5.1, its mass error is far from zero. The other values of $\boldsymbol{\omega}$ have many local maxima and minima. Figs. 5.2.2.3 and 5.2.2.4 show that these relate to pressure grid point crossings and the internal energy grid point crossing corresponds to an inflection point on the mass error curves which can be seen from the fluctuations in $\omega$ shown in Fig. 5.2.2.5. From about 0.05s to 0.2s, where neither pressure nor energy grid points are crossed, the mass error plots are relatively smooth. 
Throughout the transient, no clear best value of $\omega$ emerges. Note that because $\boldsymbol{\omega}=\mathbf{- 2 . 0}$ takes pressure into account, it generally performs better in this calculation than non-zero values of $\omega$.

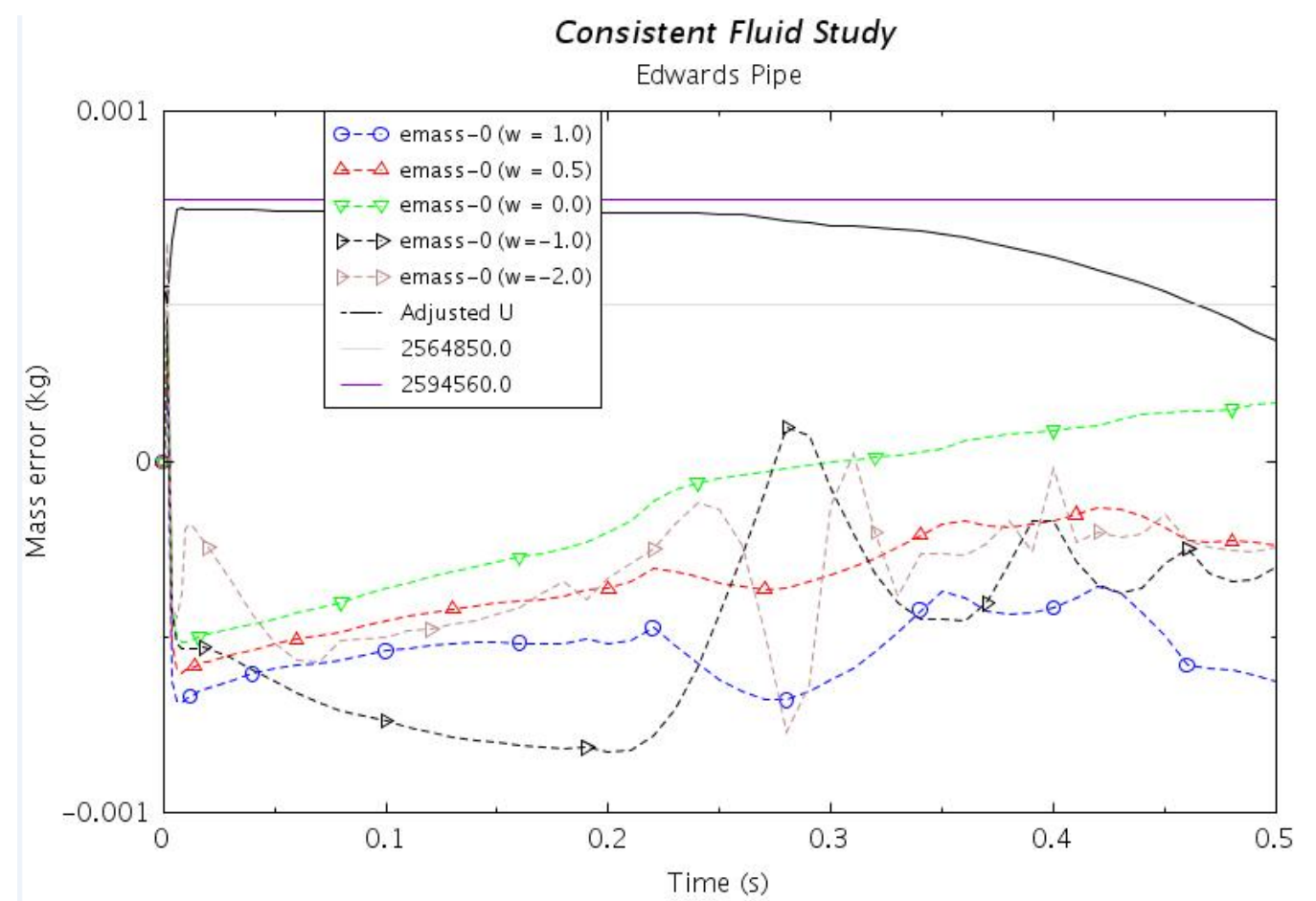

Figure 5.2.2.3a Edwards Pipe mass error. One U grid point crossed, INL card-1 options

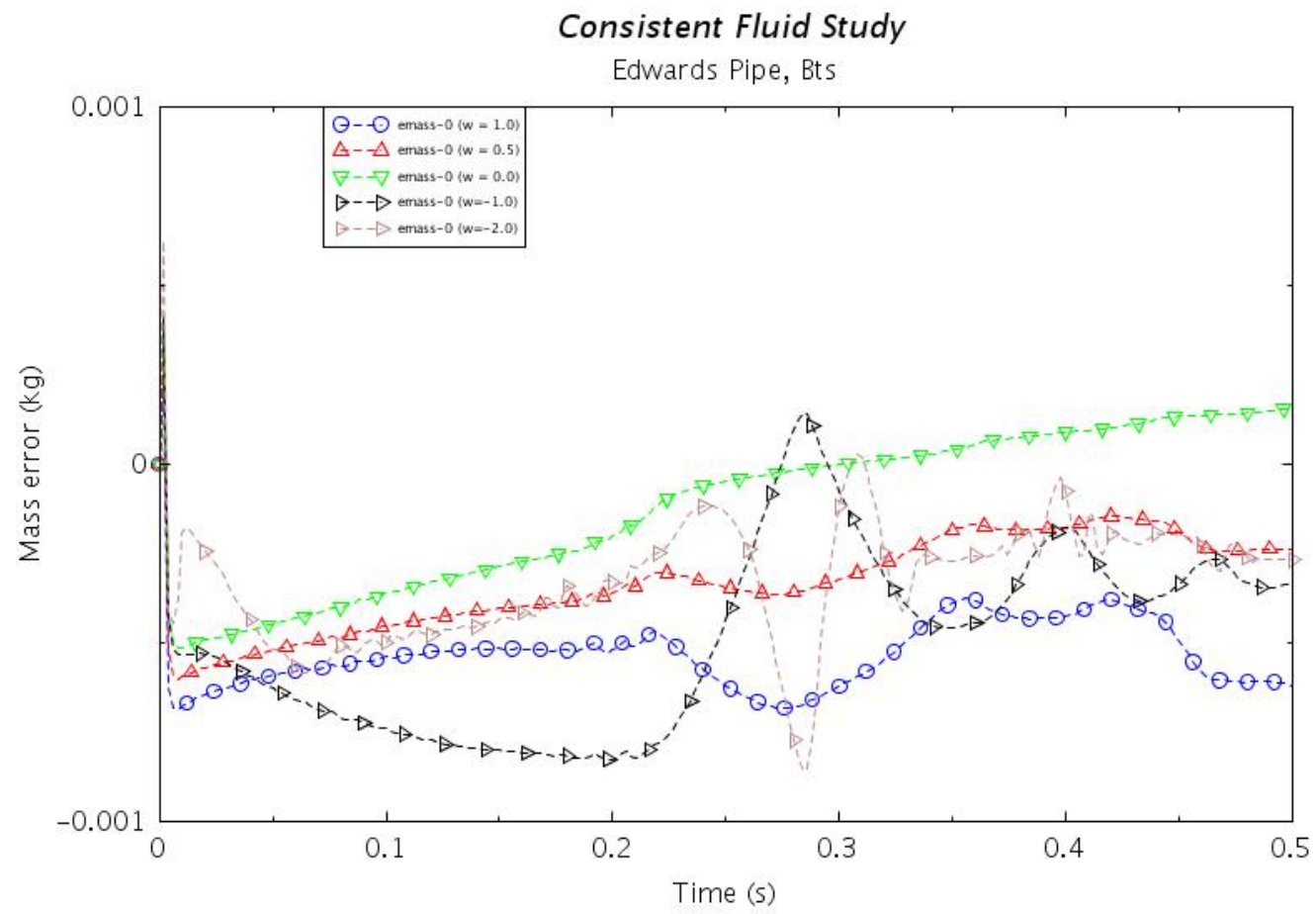

Figure 5.2.2.3b Edwards Pipe mass error. One U grid point crossed, BTS card-1 options 
All curves have a long smooth period from $0.02 \mathrm{~s}$ to $0.22 \mathrm{~s}$. The pressure line crossings again affect mass error. This is evident even for $\omega=0$ in Fig. 5.2.2.4. Figure 5.2.2.5 shows how three Omega curves are affected by pressure grid crossings; note that $\omega(P)$ is not available to users.

\section{Consistent Fluid Study}

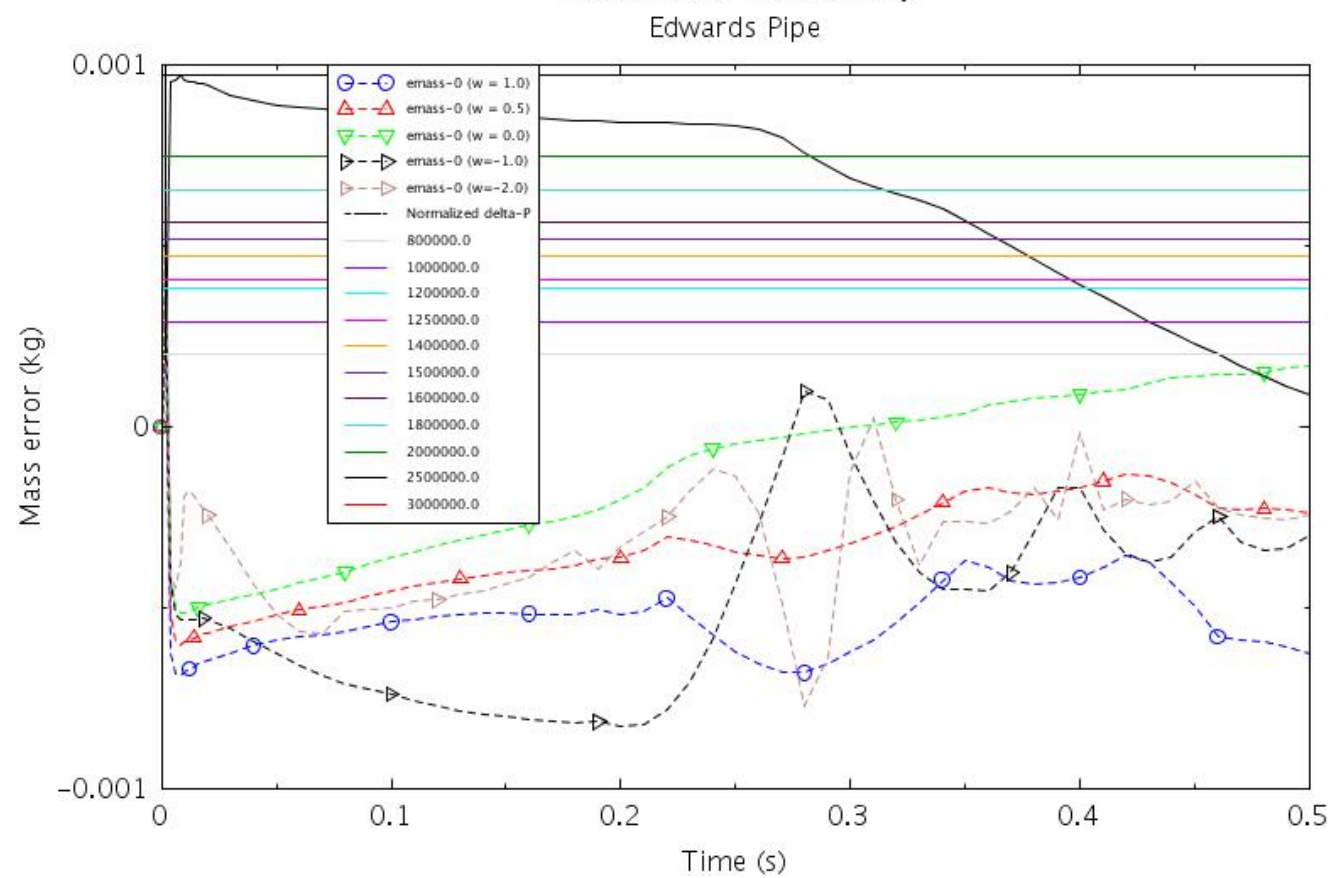

Figure 5.2.2.4 Edwards Pipe mass error. P grid points crossed, INL card-1 options

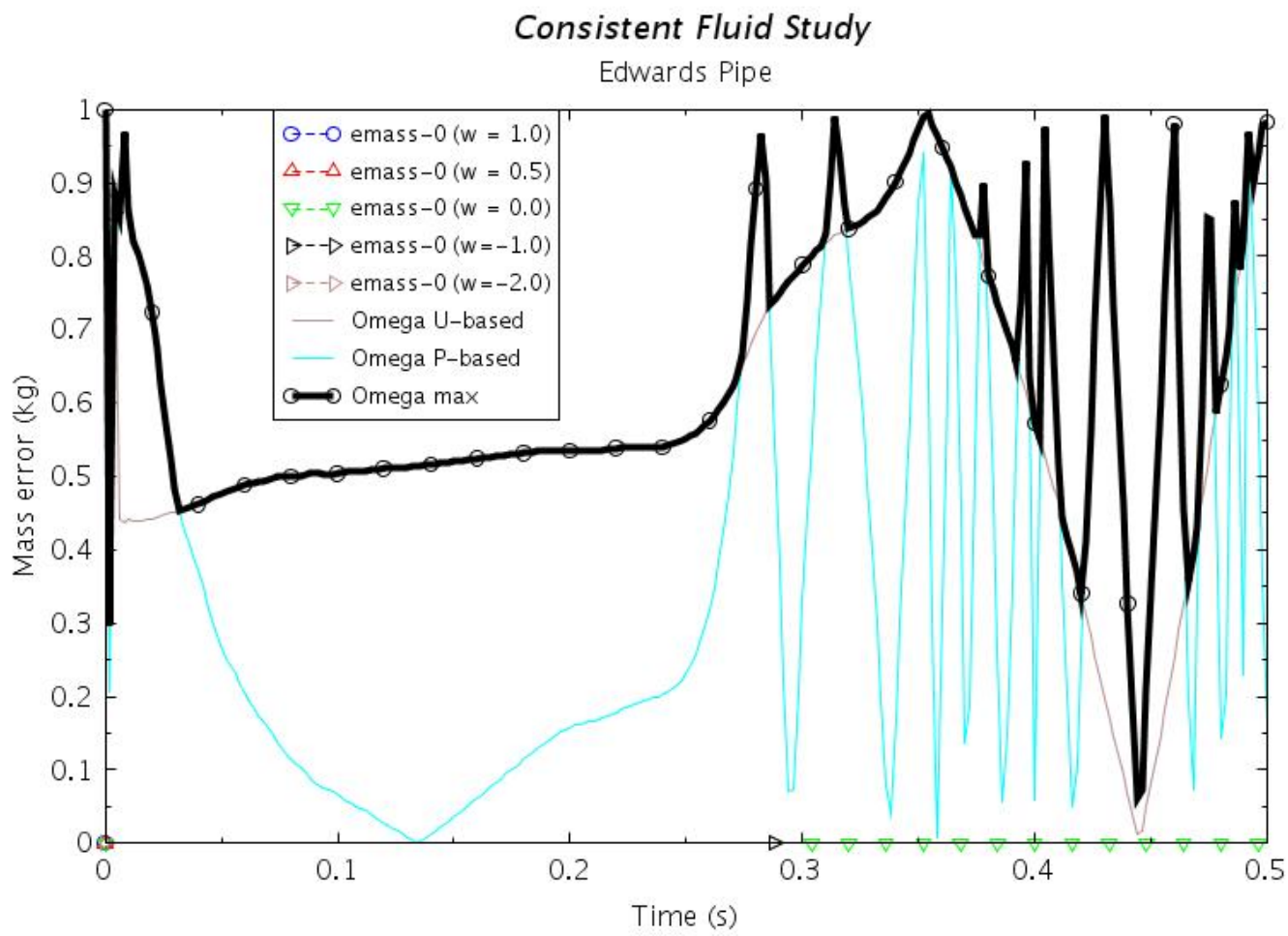

Figure 5.2.2.5 Mass error and $\omega(U), \omega(P)$, and $\max ((U), \omega(P))$ 
The Developmental Assessment graphs show no distinction in code performance for any of the omega values.

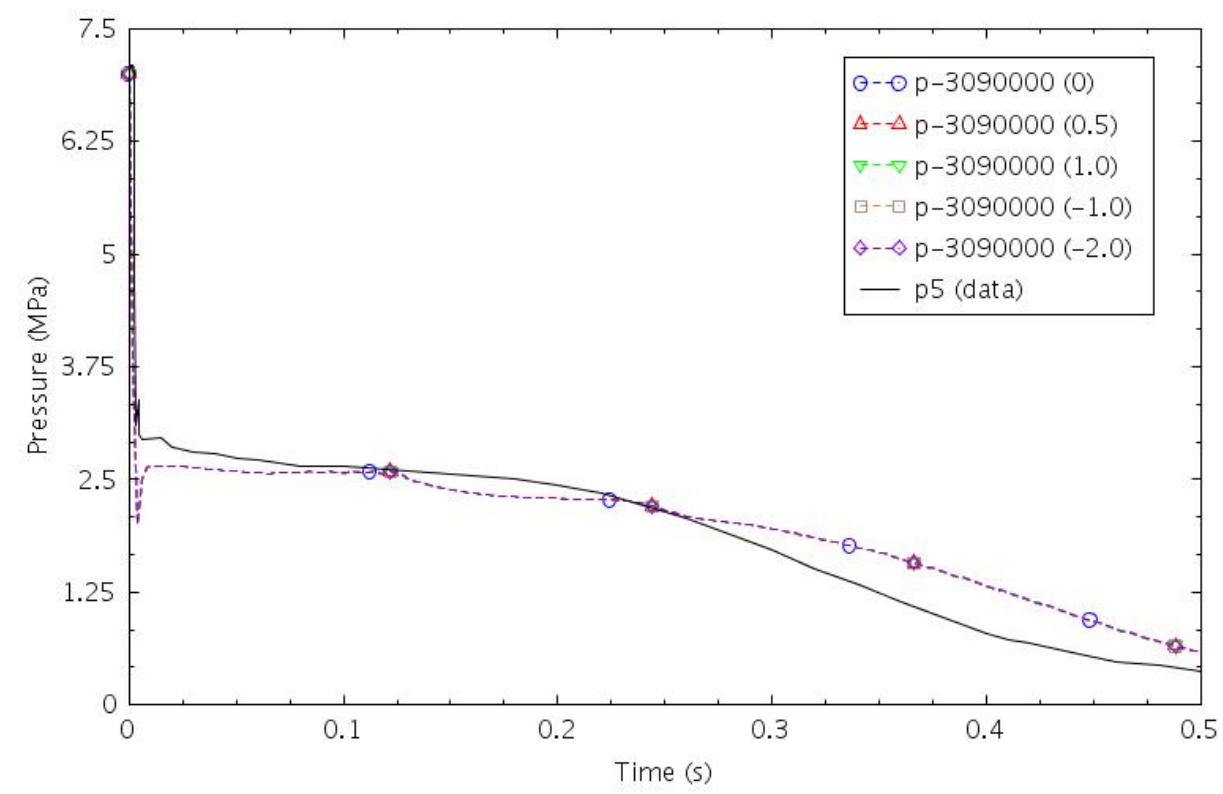

Figure 5.2.2.5 DA plot Pressure vs. time for all five omega runs.

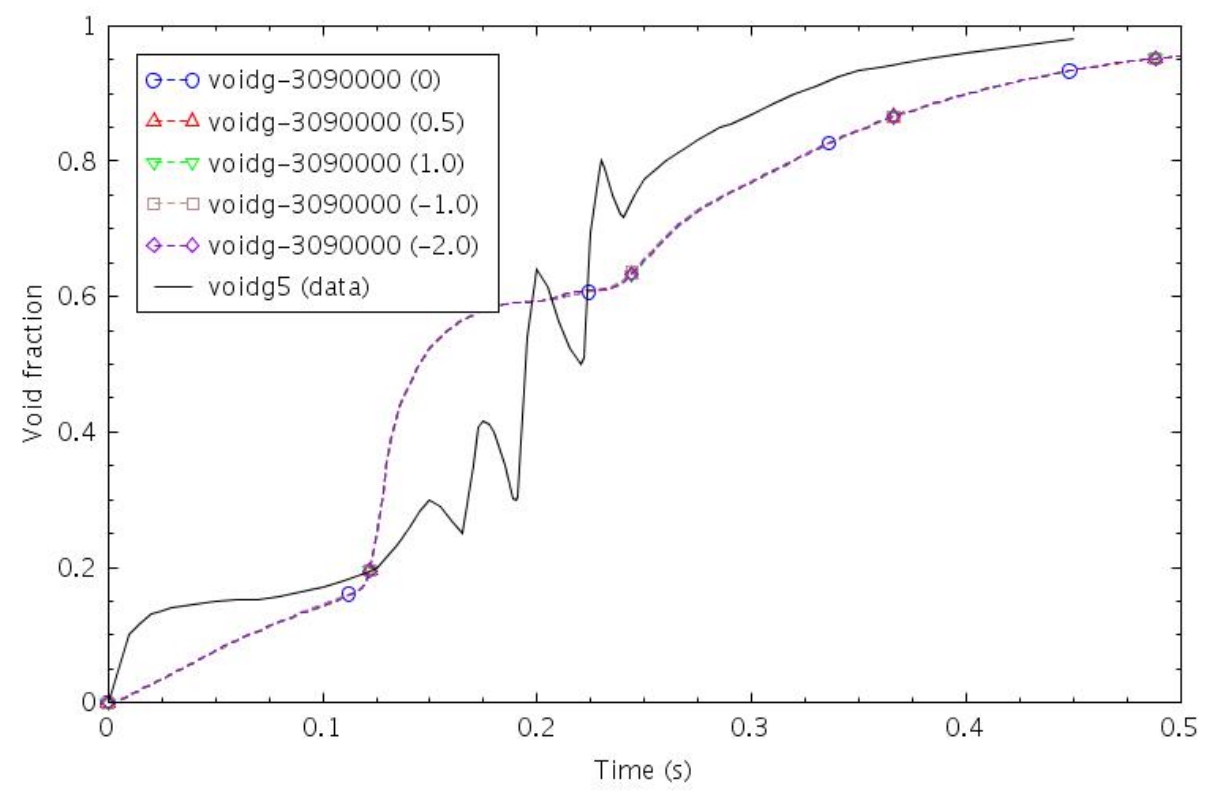

Figure 5.2.2.6 DA plot Void fraction vs. time for all five omega runs. 


\subsubsection{Typical PWR}

The typical PWR model ${ }^{[8,9]}$ of Figure 5.2.3.1 has three systems. Different values of $\boldsymbol{\omega}$ could be applied to each system. While the behavior might be optimized with separate choices that relate to the behavior of each system, a single value of $\boldsymbol{\omega}$ was used for all three systems with each separate test. This results are based on $\omega=-2,-1,0.0,0.5$, and 1.0.

\section{Testing}

Twenty-five code runs are made with the five omega-values $(1.0,0.5,0.0,-1,-2)$ and five timestep values $(\Delta t=0.1,0.05,0.01,0.005,0.001)$.

In response to discussions at the International RELAP5 User Group (IRUG) meeting, 25 more runs were made with the BTS card-1 options and plots of the timestep cuts with the different values of Omega were graphed. The timestep cuts showed very little because of the plot frequency and the fact that the code is programmed to return to DTMAX at multiples of DTMAX. Instead, Table 5.2.4.2 was created for analyzing cuts in the timesteps.

Code performance on these runs is graphed in Figs. 5.2.4.2a through 5.2.4.6b. Graphs labeled "a" use INL card-1 options while those labeled "b" use the BTS options. These are shown on the same page for comparison.

Some preliminary analysis follows.

\section{Mass error analysis}

The typical PWR problems in Figs. 5.2.3.2 through 5.2.3.6 display mass error for the five omega values when the problem is run at different levels of DTMAX. These figures do not show that one particular value of $\boldsymbol{\omega}$ is any better than any other value. The following observations are made:

- At 1200.0 seconds, mass error is about $100.0 \mathrm{~kg}$ for most values of $\mathrm{dt}$ and omega.

- In general, the mass error increases with time. The graphs have approximately the same shape except for DTMAX $=0.001$, where the mass error increases by a factor of five.

- The BTS card-1 options for DTMAX $=0.1$ cause thermodynamic property failures for $\omega$ values $0.5,-1.0$ and -2.0 (with $\alpha_{f} \approx 1.1 e-8, u_{f} \approx-1 e 5$ ), for $\omega=1.0$ at 1802.75 , and for $\omega=0.0$ at 2661.1 for nonconvergence of noncondensable mix.

- With the BTS card-1 options, the mass error decreased with decreasing dt and resulted in much less mass error than the INL options at smaller time steps.

- Generally for each choice of DTMAX, several omega values produced less mass error than the default value, $\omega=1.0$; however, the best choice varied with DTMAX.

No examination of the pressure and internal energy grid line crossings was performed. It is much more complex in this problem because there are three systems with many volumes within a system exhibiting different behavior, particularly in the primary.

\section{Timestep reduction analysis}

Table 5.2.4 compares omega values in the various timestep groups with the INL card-1 options only. It indicates that the $\omega$ values of 0.5 and -1 produce the fewest timestep cuts and never have the greatest number. The default value, $\omega=1.0$, produces the most timestep cuts and never the fewest among timestep groups. The value, $\omega=0.0$, was never the best or worst in any group.

Table 5.2.4 also shows a runtime column measured in seconds. The values $\omega=1.0$ and $\omega$ $=0.0$ took the longest every time, regardless of INL or BTS options, except for once by $\omega=-2.0$. Although timing fluctuates somewhat randomly as competing processes activate on the compute platform, it is indicative. 


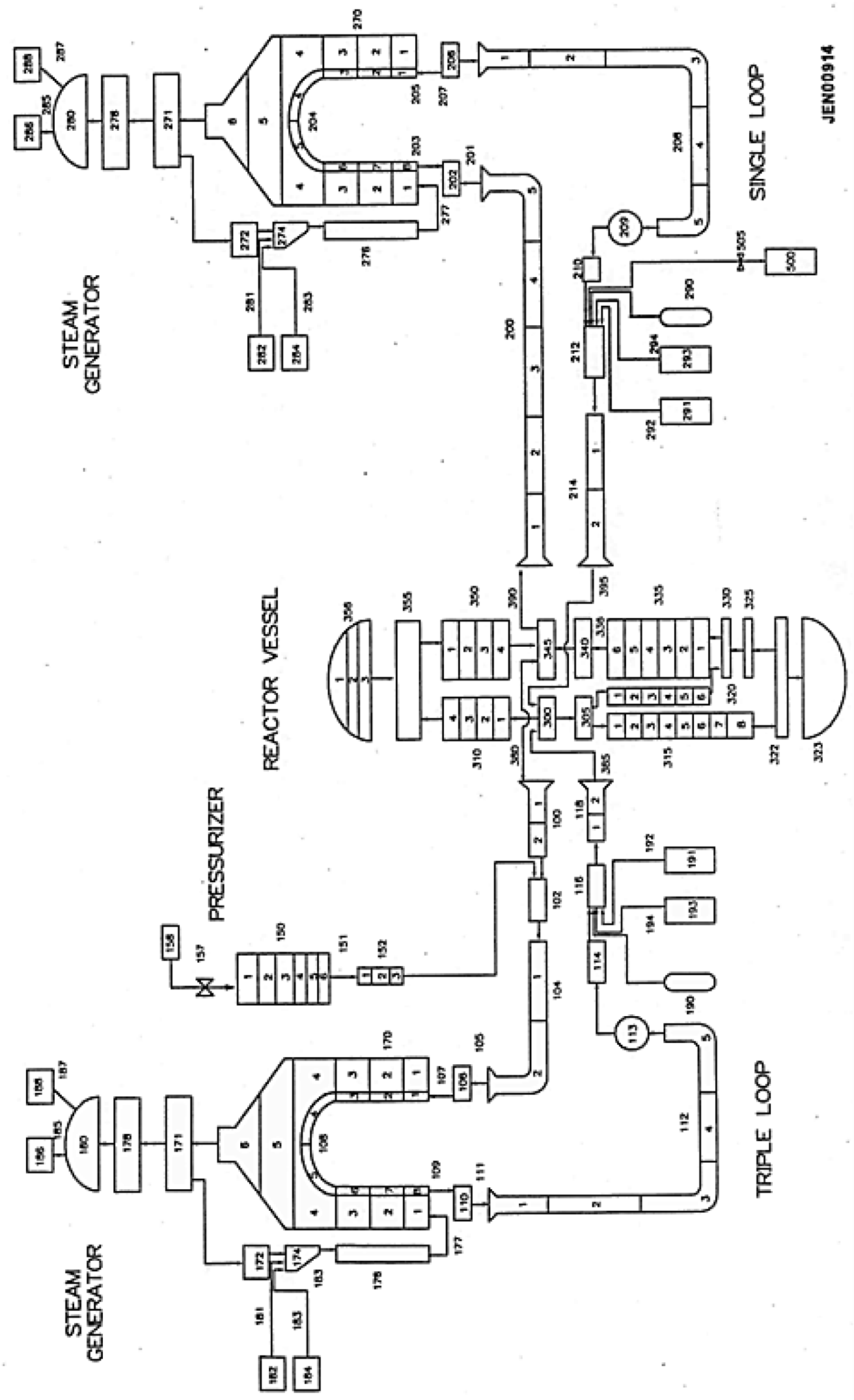

Figure 5.2.4.1 Typical PWR, Nodalization Diagram 


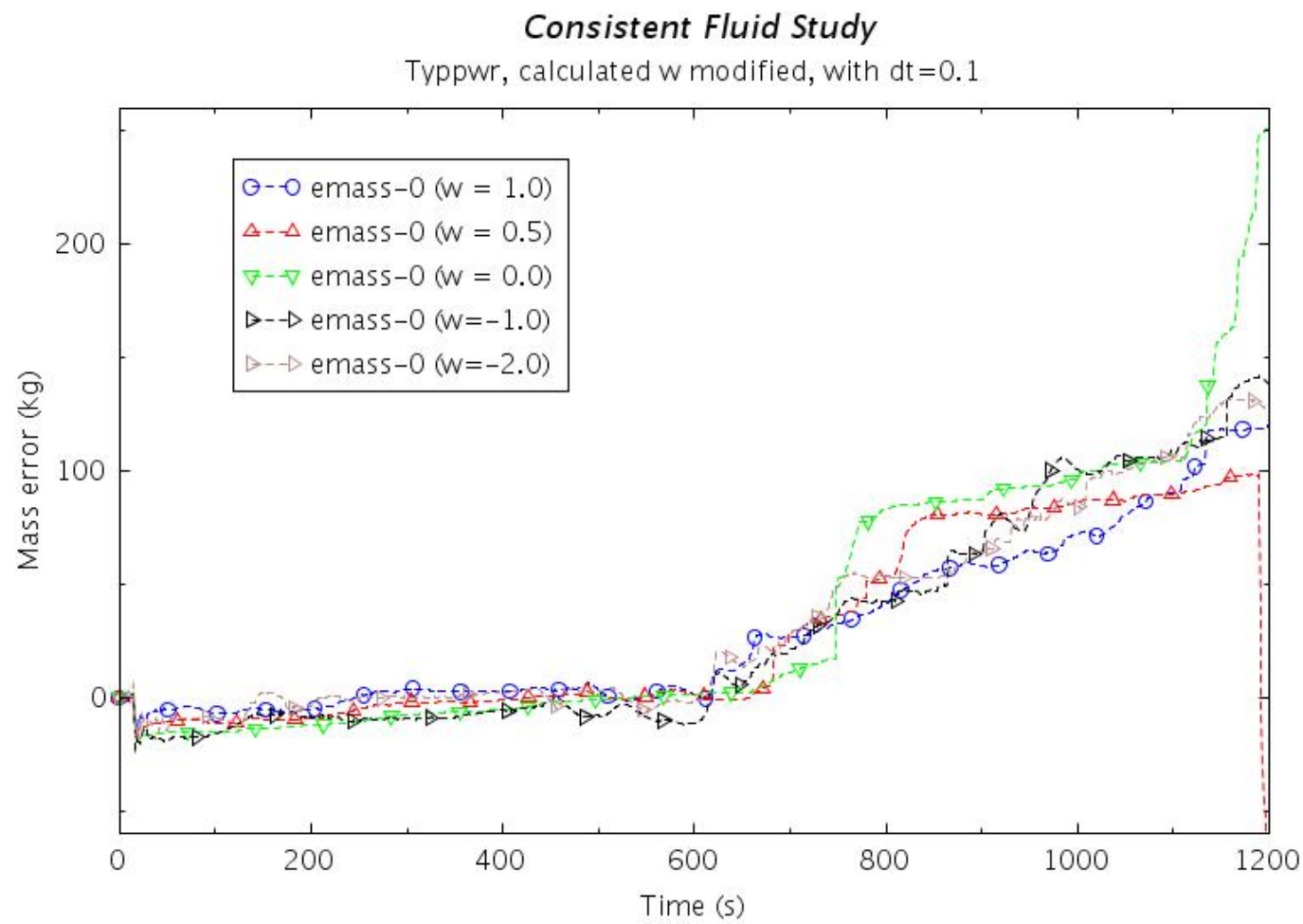

Figure 5.2.4.2a Mass error for various $\omega$ values with $\Delta t=0.1$, INL card-1 options

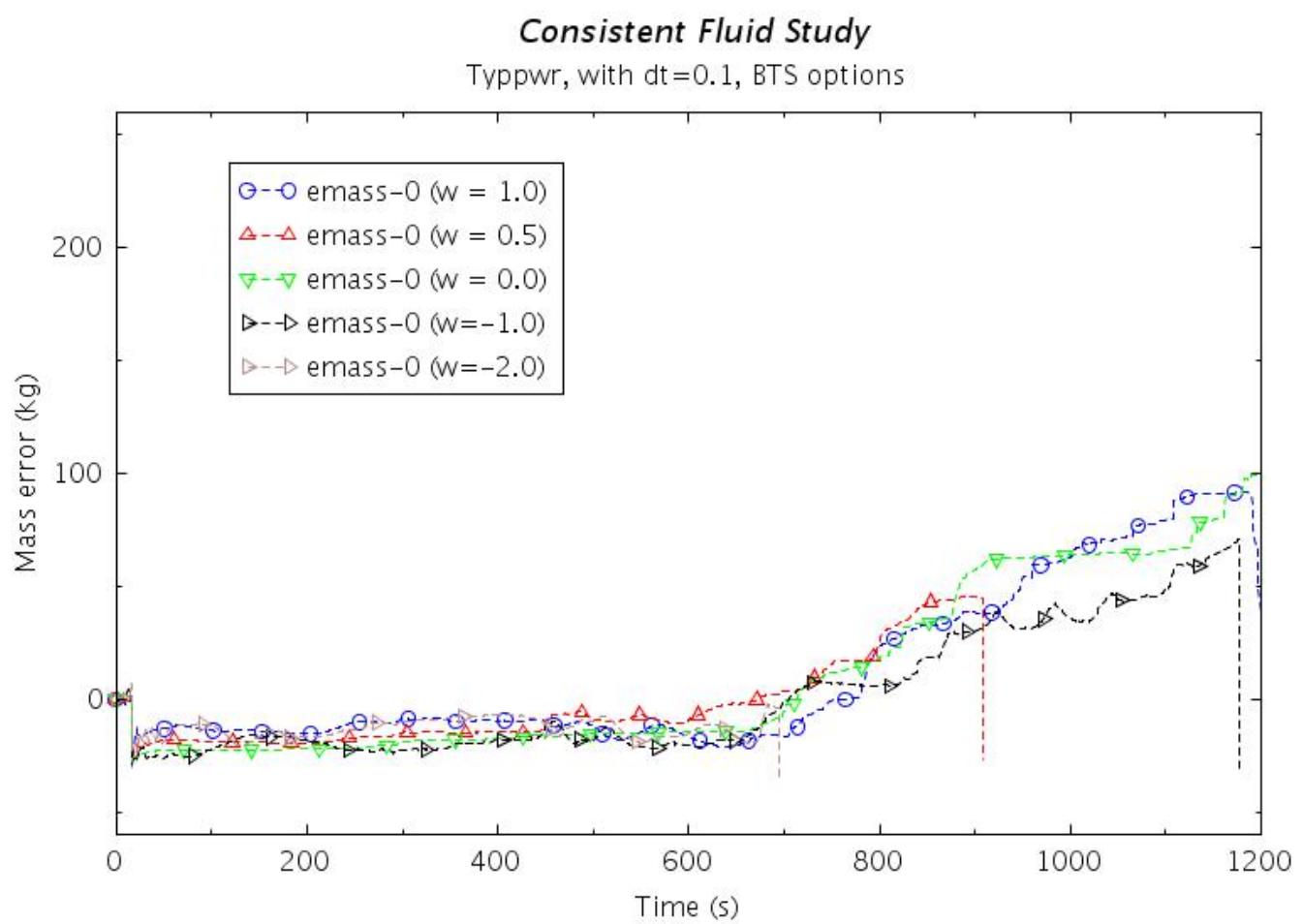

Figure 5.2.4.2b Mass error for various $\omega$ values with $\Delta t=0.1$, BTS op 


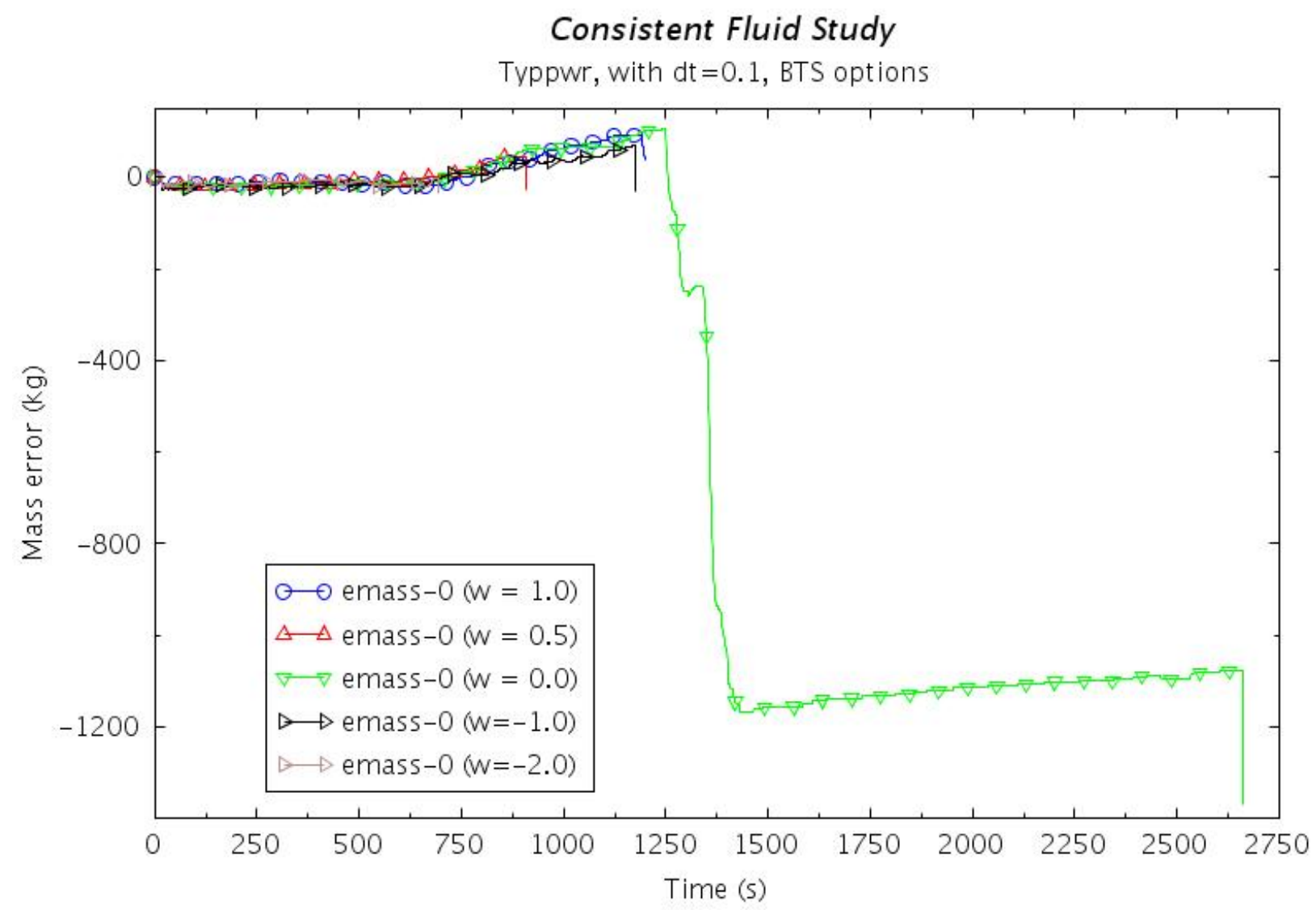

Figure 5.2.4.2c Mass error $\Delta \mathrm{t}=0.1$ and $\mathrm{BTS}$ options run until failure. 


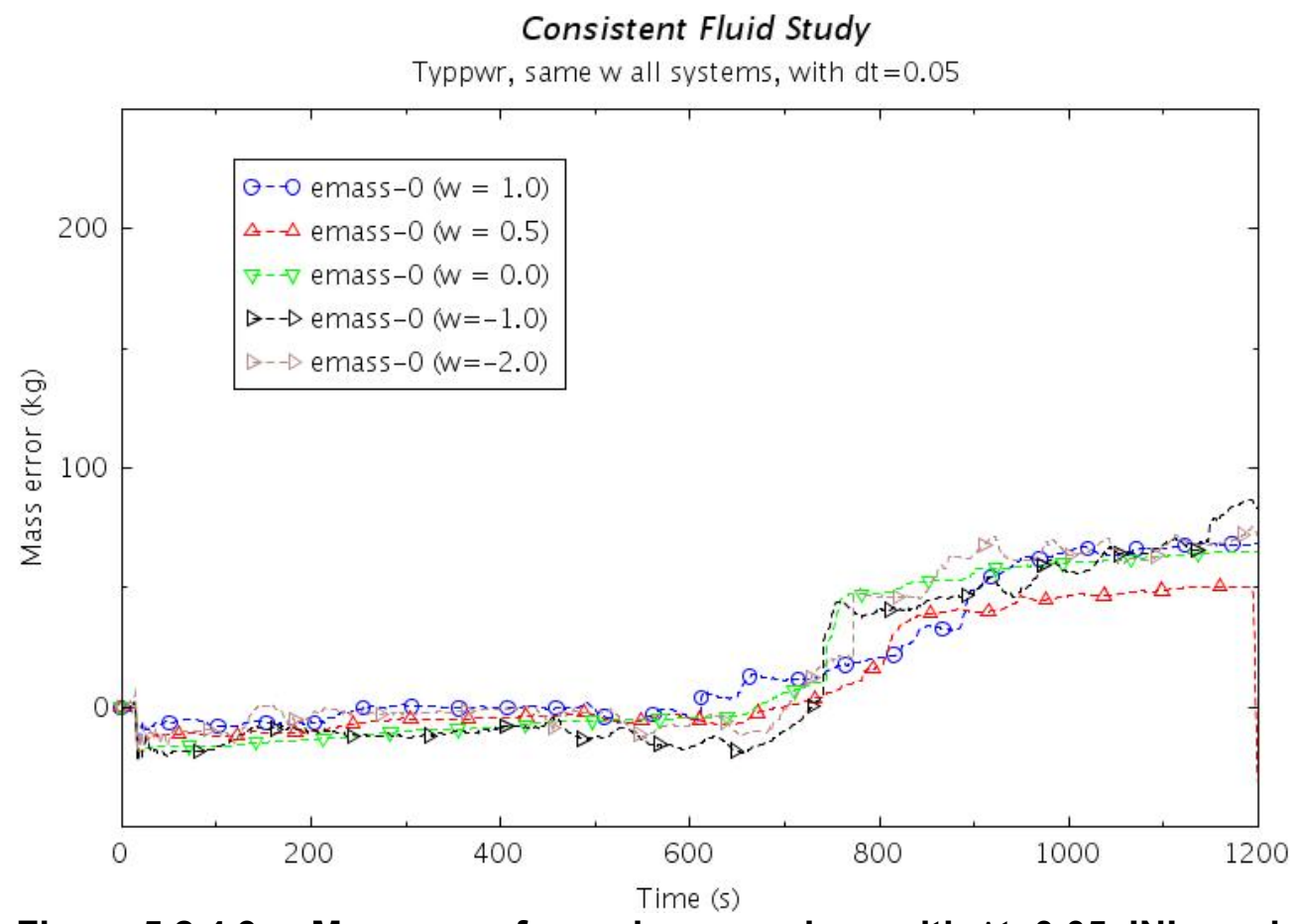

Figure 5.2.4.3a Mass error for various $\omega$ values with $\Delta t=0.05$, INL card-1 options

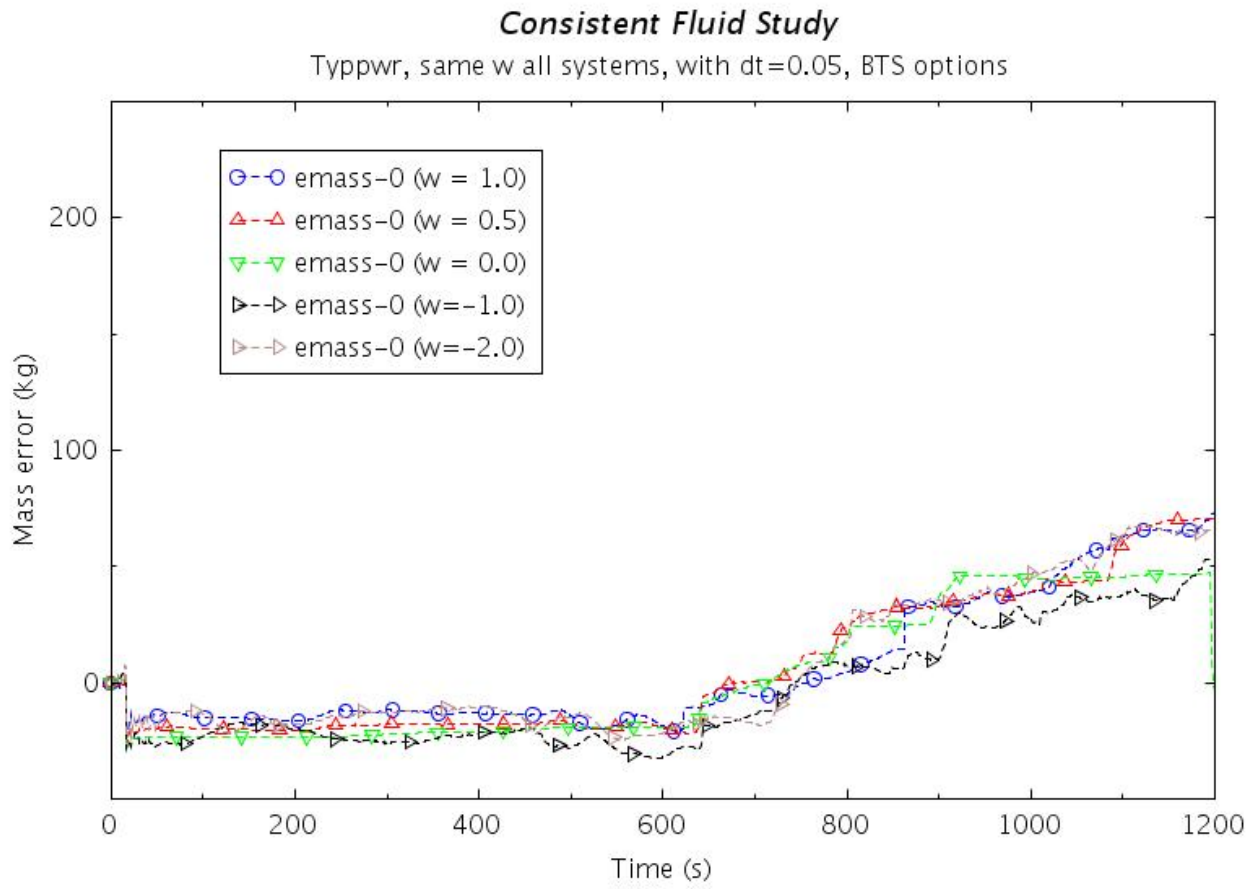

Figure 5.2.4.3b Mass error for various $\omega$ values with $\Delta t=0.05$, BTS options 


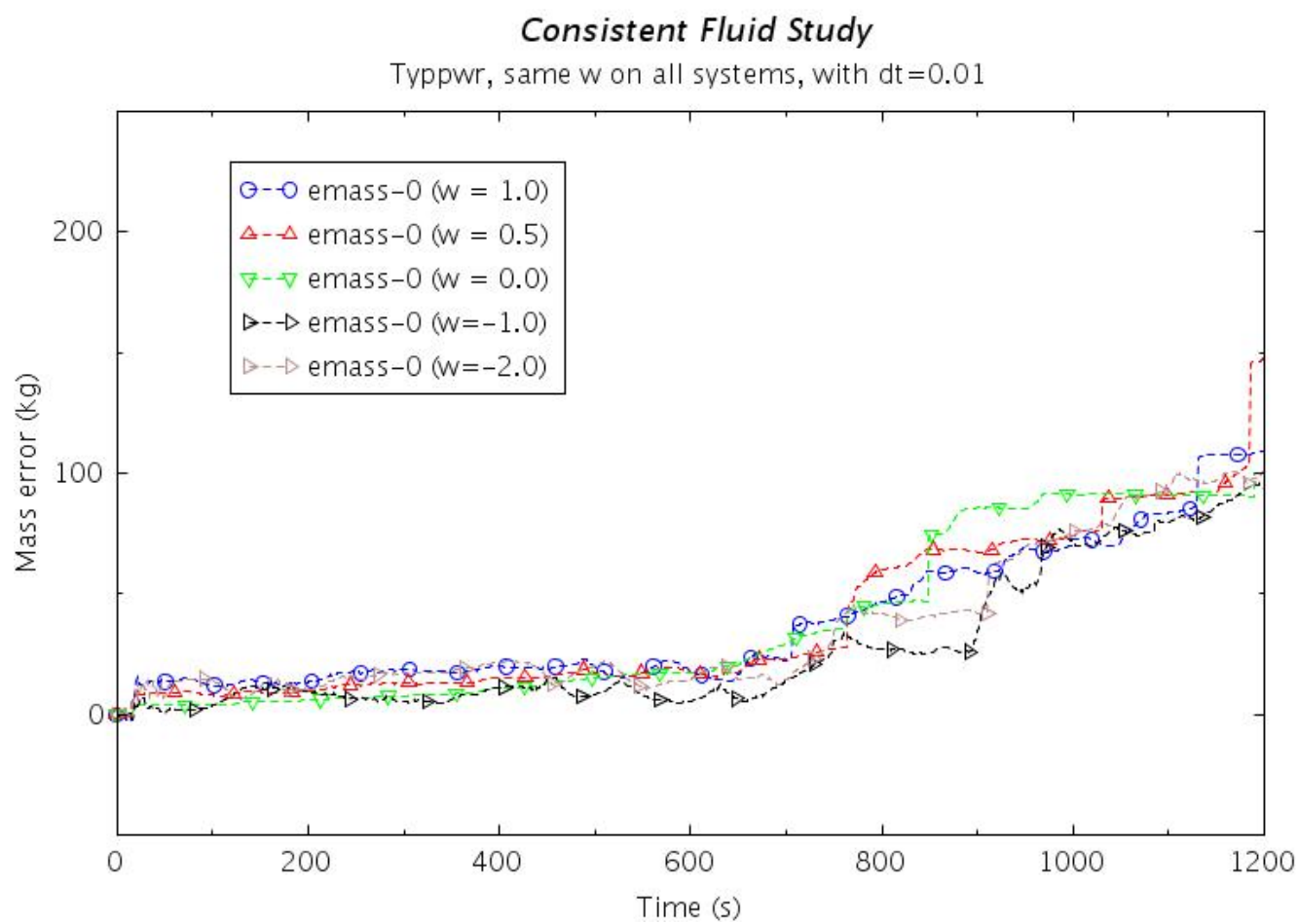

Figure 5.2.4.4a Mass error for various $\omega$ values with $\Delta t=0.01$, INL card-1 options

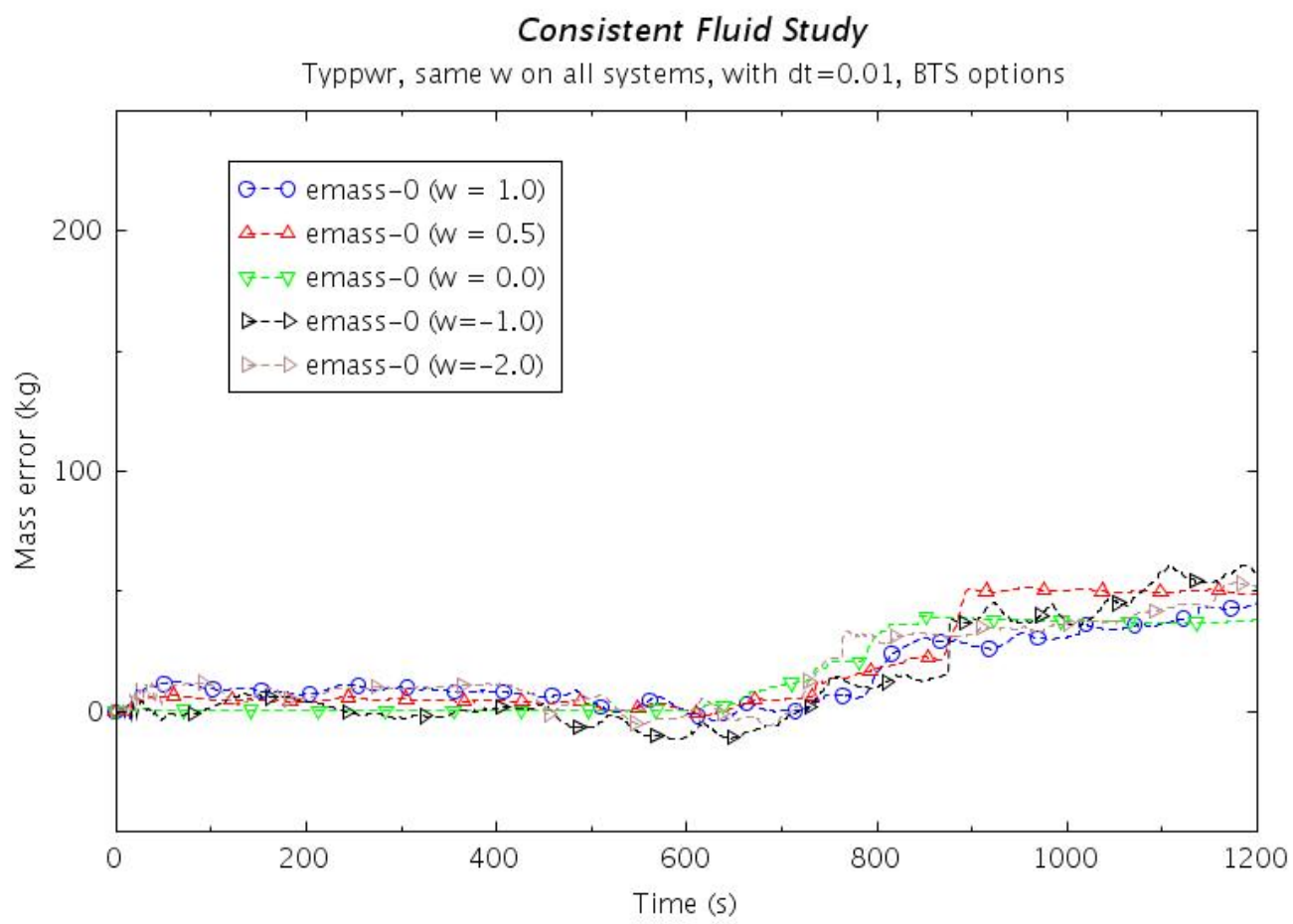

Figure 5.2.4.4b Mass error for various $\omega$ values with $\Delta t=0.01$, BTS options 


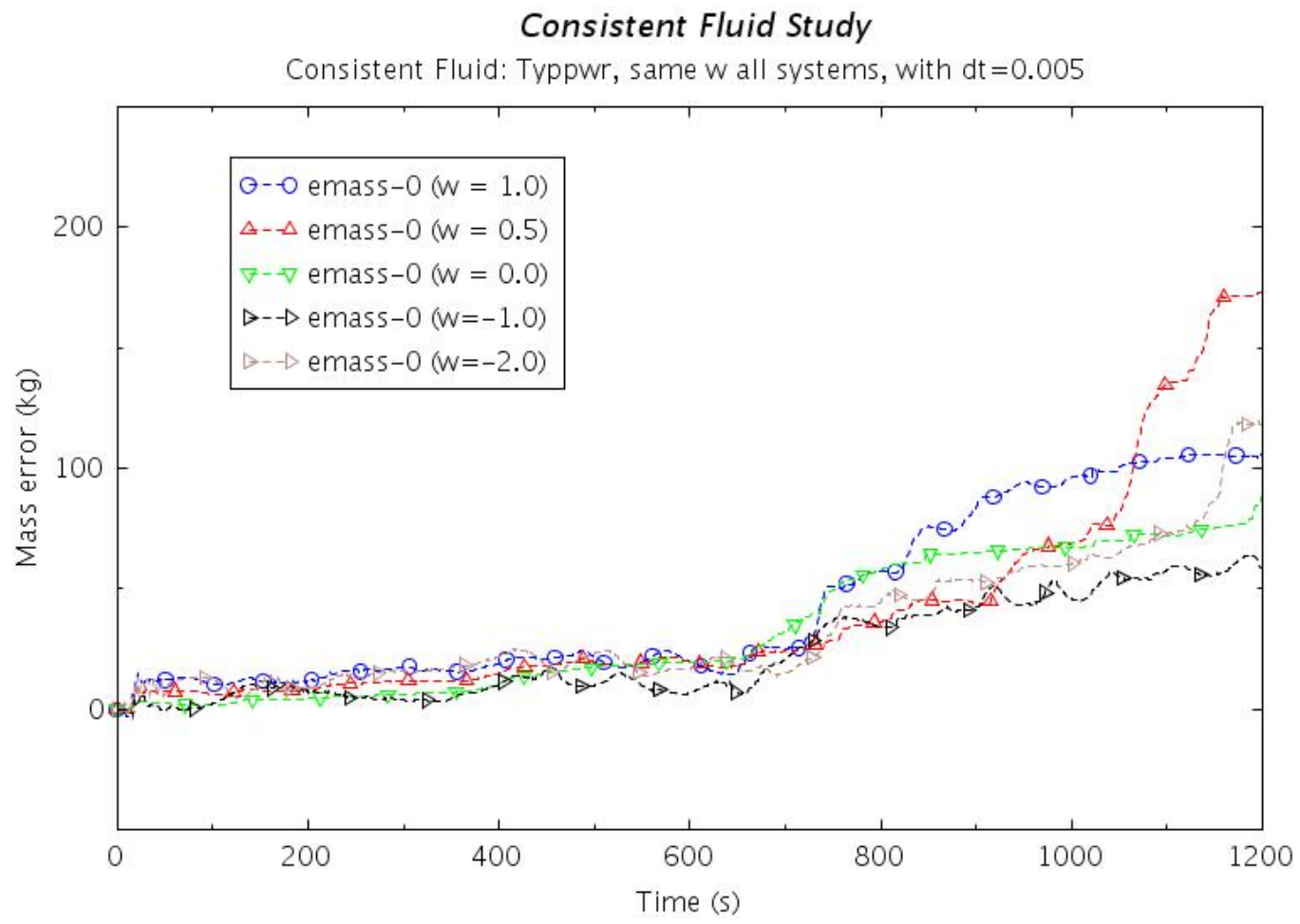

Figure 5.2.4.5a Mass error for various $\omega$ values with $\Delta t=0.005$, INL card-1 options

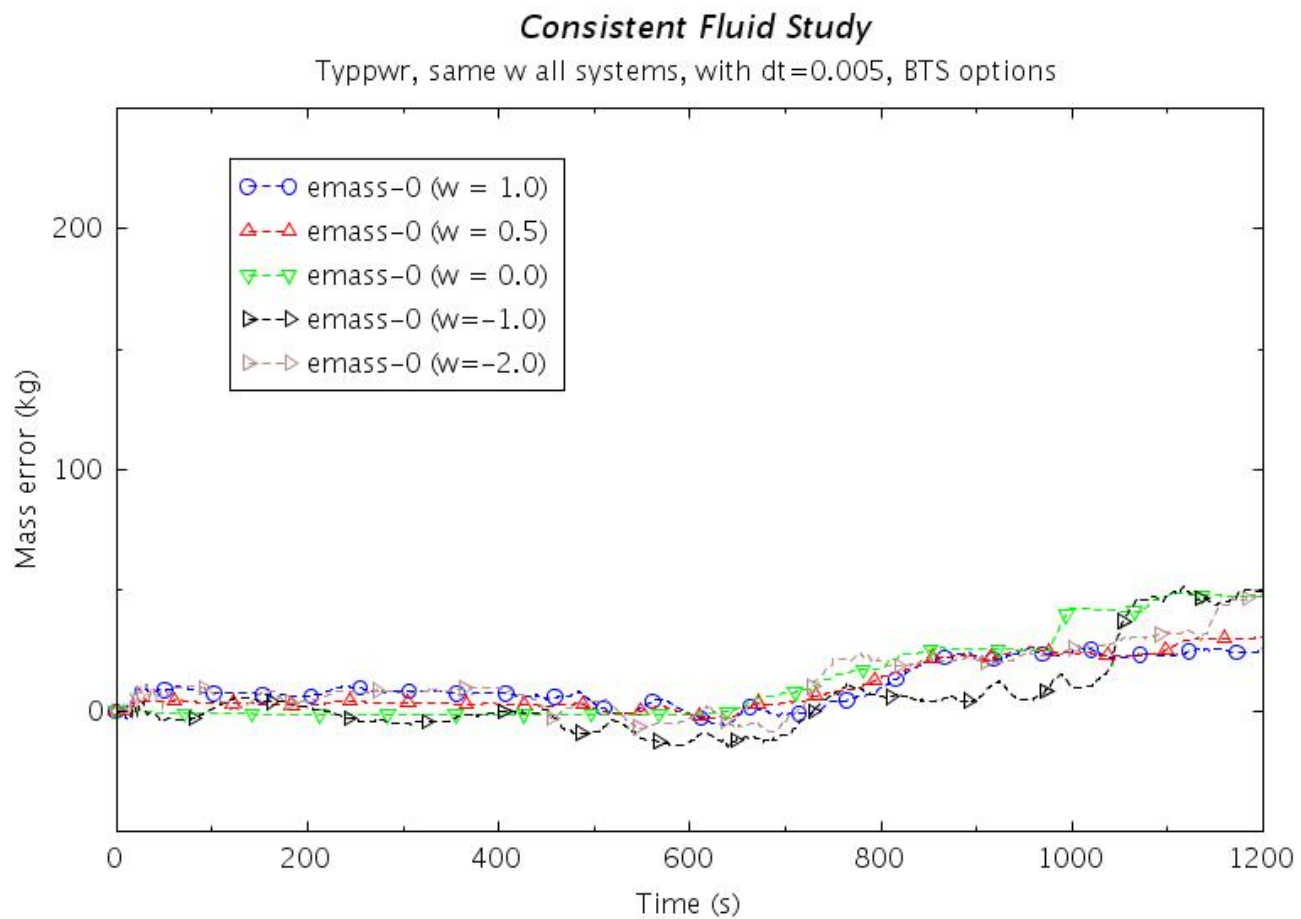

Figure 5.2.4.5b Mass error for various $\omega$ values with $\Delta t=0.005$, BTS options 
Consistent Fluid Study

Typpwr, same $w$ on all systems, with $\mathrm{dt}=0.001$

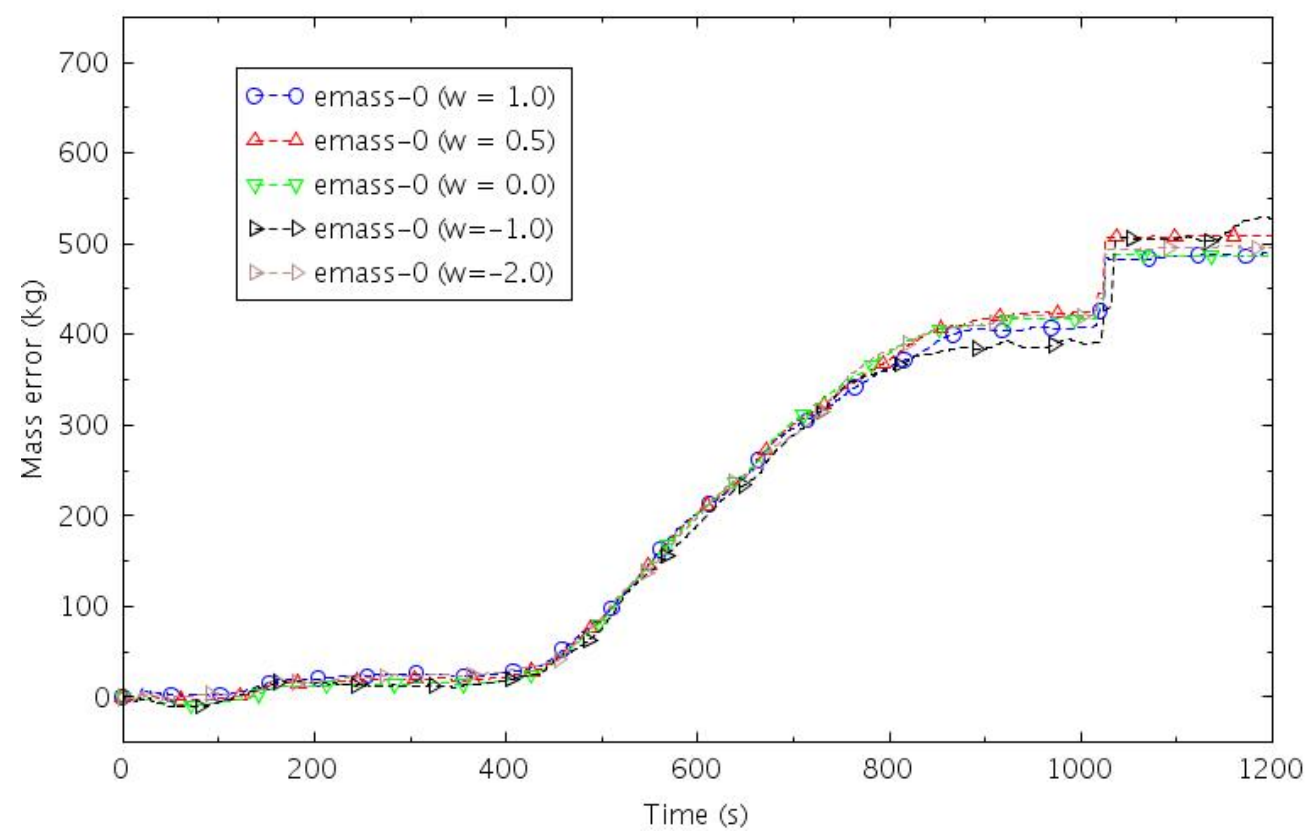

Figure 5.2.4.6a Mass error for various $\omega$ values with $\Delta t=0.001$, INL card-1 options

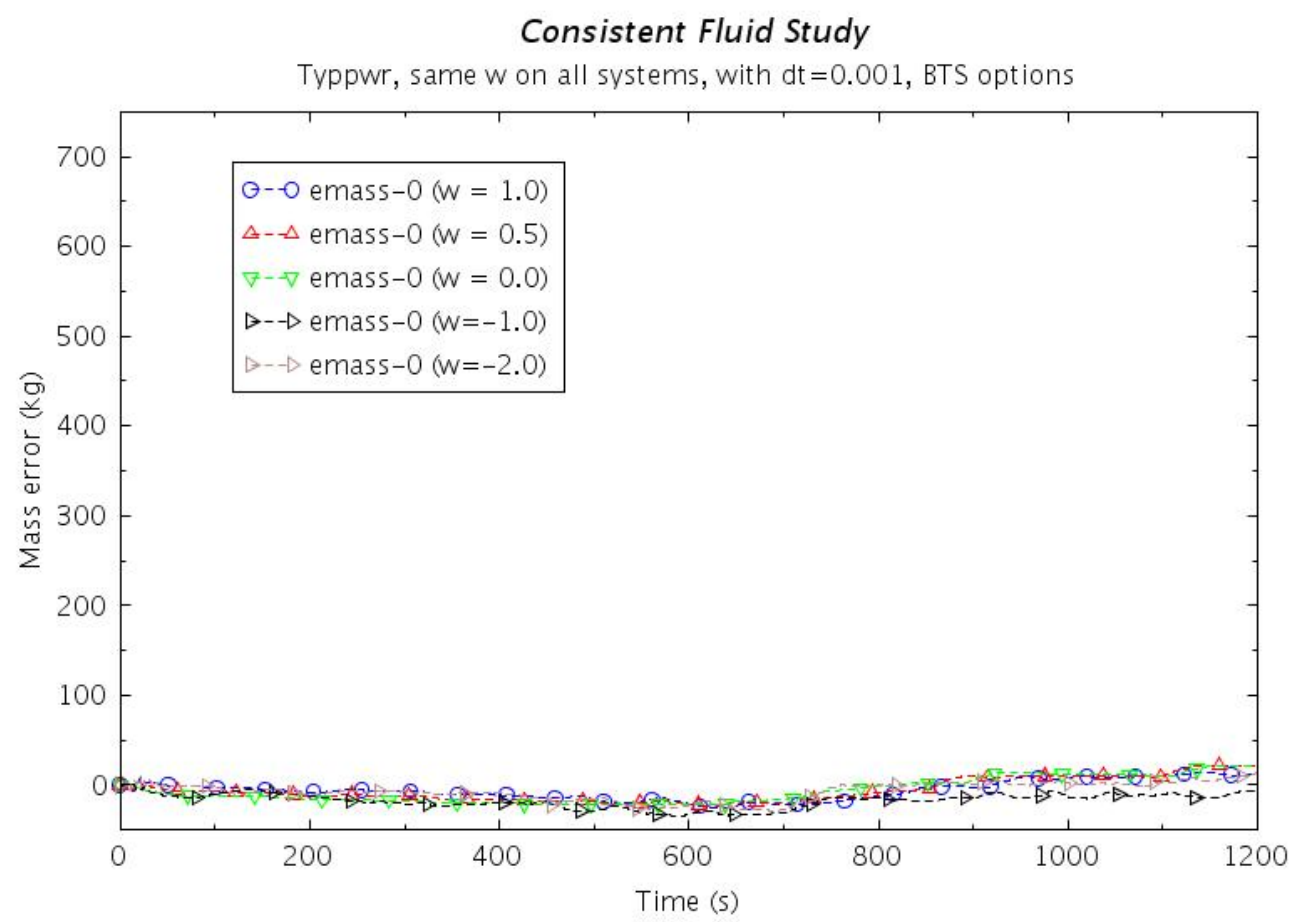

Figure 5.2.4.6b Mass error for various $\omega$ values with $\Delta t=0.001$, BTS options 
Table 5.2.4 compares failed time steps for each of these 25 runs (pairings of $\omega=1.0,0.5$, $0.0,-1.0,-2.0$ and $\mathrm{dt}=0.1,0.05,0.01,0.005,0.001)$. The "scaled" column divides the ratio by the average of the 5 ratios for that maximum time step size. The lowest scaled value for each $\mathrm{dt}$-group is shown in bold; the largest is italicized and shaded. Default-RELAP5-3D $(\omega=1.0)$ has the highest failed timestep rate in three of the groups. The lowest failed timestep ratio occurs twice for $\omega=0.5$, twice for $\omega=-1$, and once for $\omega=-2$. Never does $\omega=0.0$ distinguish itself as best or worst.

Table 5.2.4 Comparing Failed time step occurrence frequency

\begin{tabular}{|c|c|c|c|c|c|c|c|}
\hline Problem & Input $\omega$ & Fails & Advances & Ratio & Scaled & INL Time & BTS Time \\
\hline typdt1 & $\boldsymbol{\omega}=1.0$ & 5643 & 12000 & 0.47025 & 0.995063 & 22.3990 & TH prop err \\
\hline \multirow[t]{4}{*}{$\mathrm{dt}=0.1 \mathrm{~s}$} & $\boldsymbol{\omega}=0.5$ & 5798 & 12000 & 0.48317 & 1.022395 & 22.4710 & TH prop err \\
\hline & $\omega=0.0$ & 5548 & 12000 & 0.46233 & 0.978311 & 22.2350 & NC mix err \\
\hline & $\boldsymbol{\omega}=-1$ & 5863 & 12000 & 0.48858 & 1.033856 & 22.3790 & TH prop err \\
\hline & $\boldsymbol{\omega}=-2$ & 5503 & 12000 & 0.45858 & 0.970376 & 23.6440 & TH prop err \\
\hline \multicolumn{8}{|l|}{ typdt05 } \\
\hline \multirow[t]{5}{*}{$\mathrm{dt}=0.05 \mathrm{~s}$} & $\boldsymbol{\omega}=1.0$ & 5644 & 24000 & 0.23517 & 1.143158 & 38.1480 & 36.5520 \\
\hline & $\boldsymbol{\omega}=0.5$ & 4660 & 24000 & 0.19417 & 0.943855 & 37.0890 & 37.4900 \\
\hline & $\boldsymbol{\omega}=0.0$ & 5517 & 24000 & 0.22988 & 1.117435 & 37.7910 & 38.4650 \\
\hline & $\boldsymbol{\omega}=-1$ & 4167 & 24000 & 0.17363 & 0.844001 & 37.0330 & 38.3440 \\
\hline & $\omega=-2$ & 4698 & 24000 & 0.19575 & 0.951551 & 37.6790 & 37.1480 \\
\hline \multicolumn{8}{|l|}{ typdt01 } \\
\hline \multirow[t]{5}{*}{$\mathrm{dt}=0.01 \mathrm{~s}$} & $\boldsymbol{\omega}=1.0$ & 4205 & 120000 & 0.03504 & 0.893350 & 163.782 & 166.709 \\
\hline & $\omega=0.5$ & 3417 & 120000 & 0.02848 & 0.725940 & 161.273 & 163.872 \\
\hline & $\boldsymbol{\omega}=0.0$ & 5019 & 120000 & 0.04183 & 1.066284 & 164.995 & 165.386 \\
\hline & $\boldsymbol{\omega}=-1$ & 5386 & 120000 & 0.04488 & 1.144253 & 164.856 & 162.293 \\
\hline & $\boldsymbol{\omega}=-2$ & 5508 & 120000 & 0.04590 & 1.170172 & 164.265 & 165.192 \\
\hline \multicolumn{8}{|l|}{ typdt005 } \\
\hline \multirow[t]{5}{*}{$\mathrm{dt}=0.005 \mathrm{~s}$} & $\boldsymbol{\omega}=1.0$ & 6294 & 240000 & 0.02623 & 1.134013 & 322.617 & 323.079 \\
\hline & $\omega=0.5$ & 6085 & 240000 & 0.02535 & 1.096357 & 319.876 & 321.441 \\
\hline & $\omega=0.0$ & 5813 & 240000 & 0.02422 & 1.047350 & 324.123 & 321.314 \\
\hline & $\boldsymbol{\omega}=-1$ & 4627 & 240000 & 0.01928 & 0.833664 & 320.208 & 322.817 \\
\hline & $\boldsymbol{\omega}=-2$ & 4932 & 240000 & 0.02055 & 0.888617 & 321.492 & 321.537 \\
\hline \multicolumn{8}{|l|}{ typdt001 } \\
\hline \multirow[t]{5}{*}{$\mathrm{dt}=0.001 \mathrm{~s}$} & $\omega=1.0$ & 10447 & 1200000 & 0.00871 & 1.070411 & 1586.39 & 1586.39 \\
\hline & $\omega=0.5$ & 8914 & 1200000 & 0.00743 & 0.913338 & 1573.01 & 1573.19 \\
\hline & $\omega=0.0$ & 9645 & 1200000 & 0.00804 & 0.988237 & 1569.71 & 1564.25 \\
\hline & $\boldsymbol{\omega}=-1$ & 9797 & 1200000 & 0.00816 & 1.003812 & 1572.23 & 1573.02 \\
\hline & $\boldsymbol{\omega}=-2$ & 9996 & 1200000 & 0.00833 & 1.024201 & 1575.68 & 1578.11 \\
\hline
\end{tabular}




\subsubsection{Moby Dick problem}

In response to discussions at the International RELAP5 User Group (IRUG) meeting, the Moby Dick problem was run because it uses noncondensables and is a DA problem.

Performed in the late 1970s at the French Atomic Energy Commission laboratories (Centre d'Etudes Nucleaires de Grenoble), Moby-Dick Air-Water Critical Flow Experiment 3141 was one of a series of experiments to study steady state, two-phase, two-component critical flow in a vertical, divergent nozzle test section. Experiment 3141 was a test with upstream nitrogen injection at low temperature and high flow rate into a low temperature inlet water flow. The test data were obtained under constant inlet flow, steady state conditions.

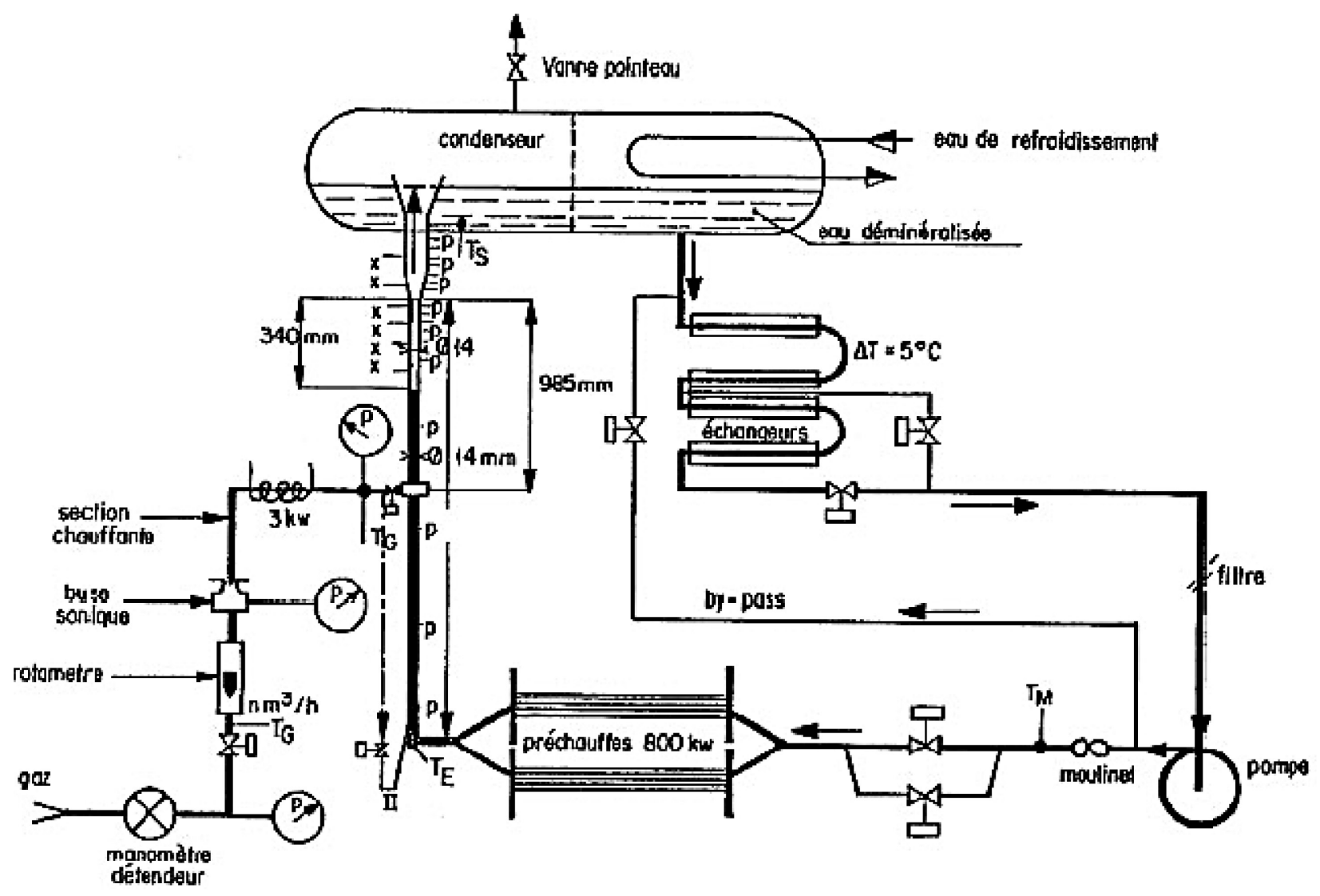

Figure 5.2.5.1 Moby Dick diagram

The nodalization diagram is given in Fig. 5.2.5.2. Time-dependent volume components are used to specify the inlet boundary pressure and temperature conditions for the water (Component 103) and gas (Component 107) injection flows; the conditions used are those in Table 4.6-1. The inlet pressure is 518.9 $\mathrm{kPa}$ for the water and $450 \mathrm{kPa}$ for the gas. The steady state liquid flow rate of $1.222 \mathrm{~kg} / \mathrm{s}$ is injected at the pipe inlet, and the steady state gas flow rate of $6.1 \times 10-3 \mathrm{~kg} / \mathrm{s}$ is injected at the inlet of pipe cell 4 . The mixture exits the test pipe section into a condenser sink volume at approximately atmospheric pressure.

Sixteen Moby Dick tests comprise the DA suite. When combined with 5 omega values, this creates 80 test cases. The naming convention is summarized in Table 5.2.5.1. A name is formed from the Base Test name concatenated with a Coupling/Advancement indicator, a period, and an Omega Abbreviation. The Coupling/Advancement indicator is "ic" for implicit coupling between heat transfer and hydrodynamics. 


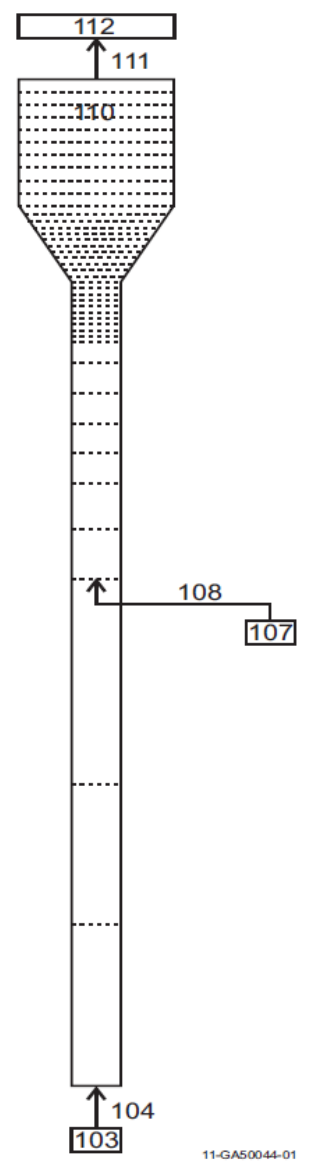

Figure 5.2.5.2 Moby Dick Nodalization diagram

These cases all use the default TPFH2O property file. To perform the study, system 120 cards were added to specify fluid $\mathrm{H} 2 \mathrm{ON}$.

Table 5.2.5.1 Major Moby Dick base case input decks

\begin{tabular}{|l|l|l|l|l|l|}
\hline Base Test & $\begin{array}{l}\text { Card-1 } \\
\text { options }\end{array}$ & $\begin{array}{l}\text { Coupling/ } \\
\text { Advance }\end{array}$ & Meaning & $\begin{array}{l}\text { Omega } \\
\text { Abbrev. }\end{array}$ & $\begin{array}{l}\text { Input } \\
\text { Omega }\end{array}$ \\
\hline Mobydck4 & none & & $\begin{array}{l}\text { Explicit-coupling, } \\
\text { semi-implicit }\end{array}$ & $\mathbf{1 p 0}$ & 1.0 \\
\hline Mobydck4c1 & 55 & $\mathbf{i c}$ & $\begin{array}{l}\text { Implicit coupling } \\
\text { Semi-implicit }\end{array}$ & $\mathbf{0 p 5}$ & 0.5 \\
\hline Mobydck4c2 & 3 & $\mathbf{n i}$ & $\begin{array}{l}\text { Nearly implicit } \\
\text { Explicit coupling }\end{array}$ & $\mathbf{0 p 0}$ & 0.0 \\
\hline Mobydck4c12 & 3 and 55 & $\mathbf{n i - c i}$ & $\begin{array}{l}\text { Nearly implicit } \\
\text { Implicit coupling }\end{array}$ & $\mathbf{m 1}$ & -1 \\
\hline & & & & $\mathbf{m 2}$ & -2 \\
\hline
\end{tabular}

Fig 5.2.5.1, from the developmental Assessment plots pressure vs. elevation for the calculation and data. It is evident that the choice of omega value has negligible effect on the pressure calculation. 


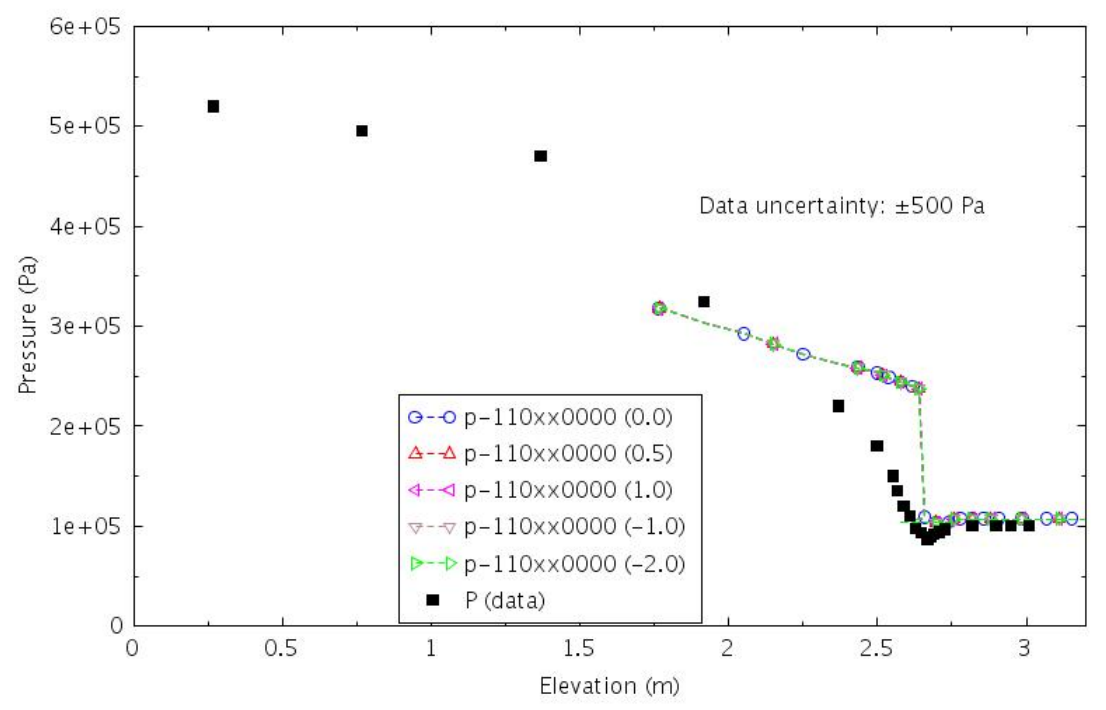

Figure 5.2.5.1 Pressure vs. elevation for Moby Dick, all five standard omega values

Table 5.2.5.2 shows four columns using INL option on the left and four on the right with BTS values. The leftmost column contains the omega values. Other columns have 20 input deck names indicating the base Moby Dick input model and type of coupling/advancement.

Table 5.2.5.2 Mass error ratio evolution, 80 cases with INL options, 80 with BTS

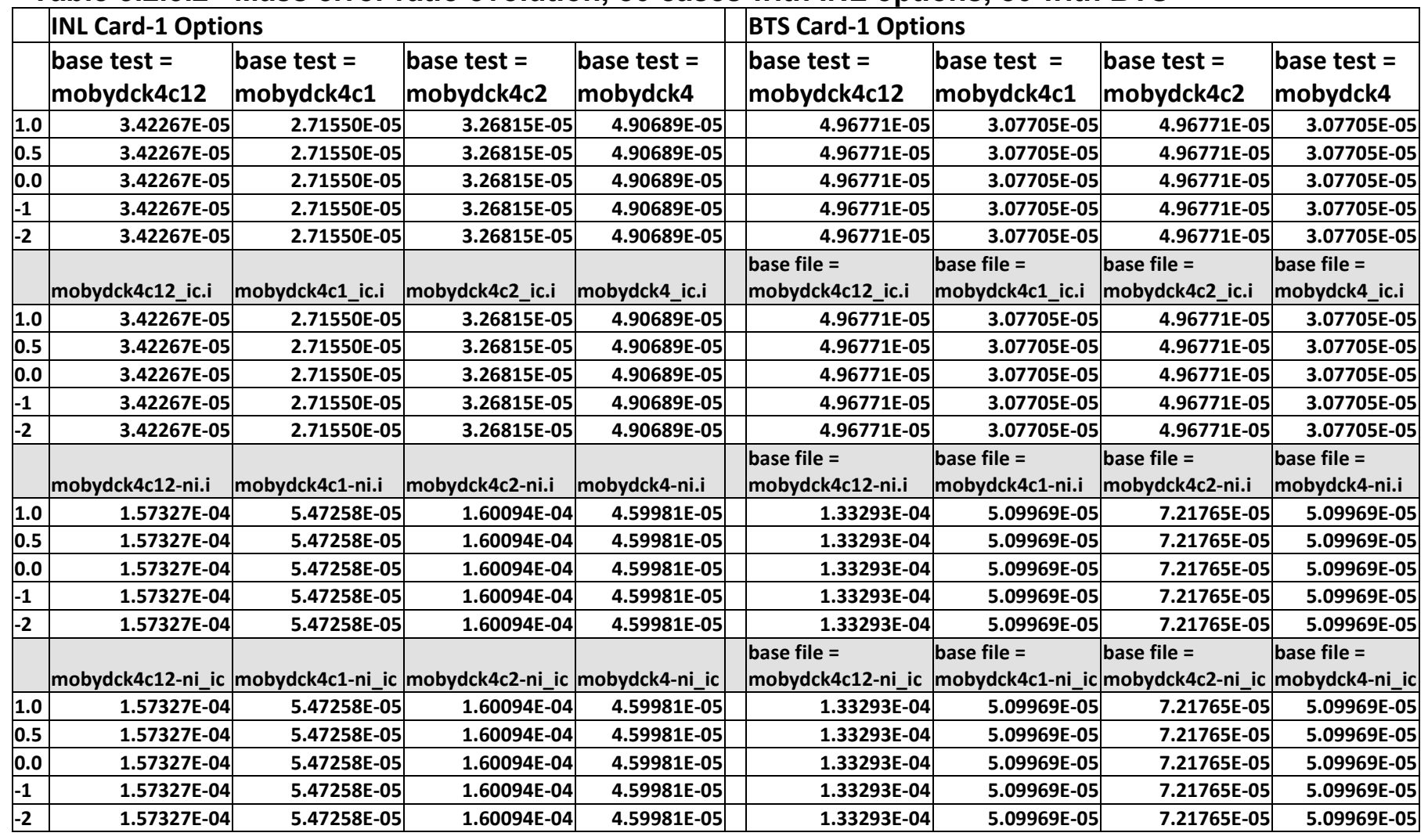




\section{Observations}

- $\quad$ The mass error with $\mathrm{H} 2 \mathrm{ON}$ was typically1.5 times lower than the mass error ratio produced with default $\mathrm{H} 2 \mathrm{O}$.

- For each of the 16 Base Test and Coupling/Advancement combinations, there were no noteworthy differences among the omega values.

- With INL options, the mass error ratio remains constant for some, not for others

- BTS options produce lower mass error ratios for the ni, ic, and ni-ic options, but for the base case, the INL options have smaller mass error.

- The only exception to this rule is mobydck4-ni_ic.

- For some base case runs with nearly implicit, the INL options have lower mass error ratio.

- The DA graph of pressure vs. elevation shows no visible differences between the five values of omega. 


\subsubsection{Mixbub}

For a more strenuous test of mass error, the Developmental Assessment problem with highest mass error ratio was exercised. The mixbub.i is a thought problem that bubbles saturated steam up through a column of saturated liquid water, Fig. 5.2.6.1. The steam flow rate increases in steps to allow quasi-steady conditions to be established. The flow rate is then increased linearly to a value high enough to entrain liquid out of the top of the column. The problem runs to 1400.0 seconds.

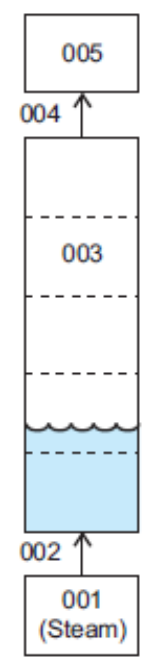

Figure 5.2.6.1 Nodalization diagram for the mixbub input model

The problem is run with both INL and BTS card-1 options and the mass error is graphed in Figs. 5.2.6.2a and 5.2.6.2b.

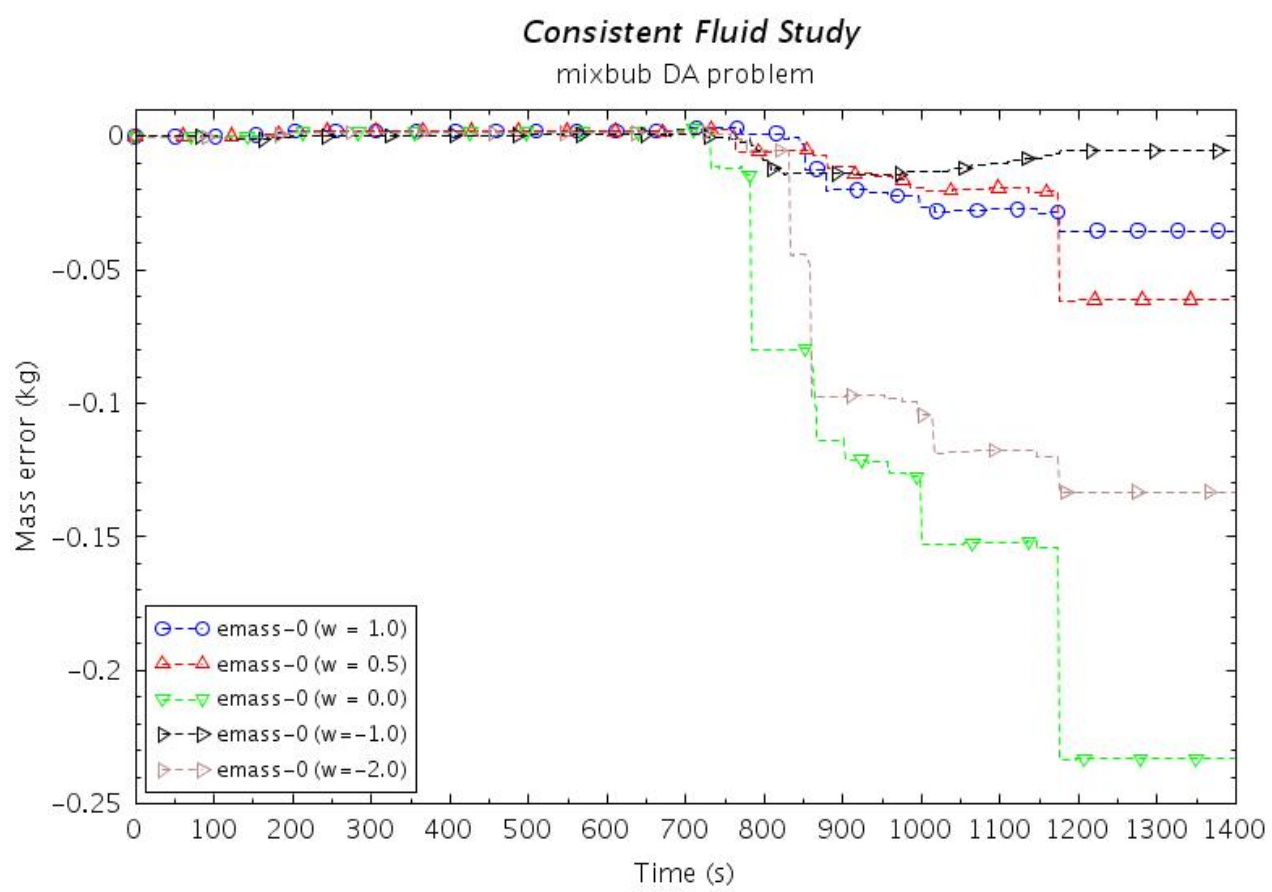


Figure 5.2.6.1a Mass error in mixbub.i for various omega values, INL card-1 options

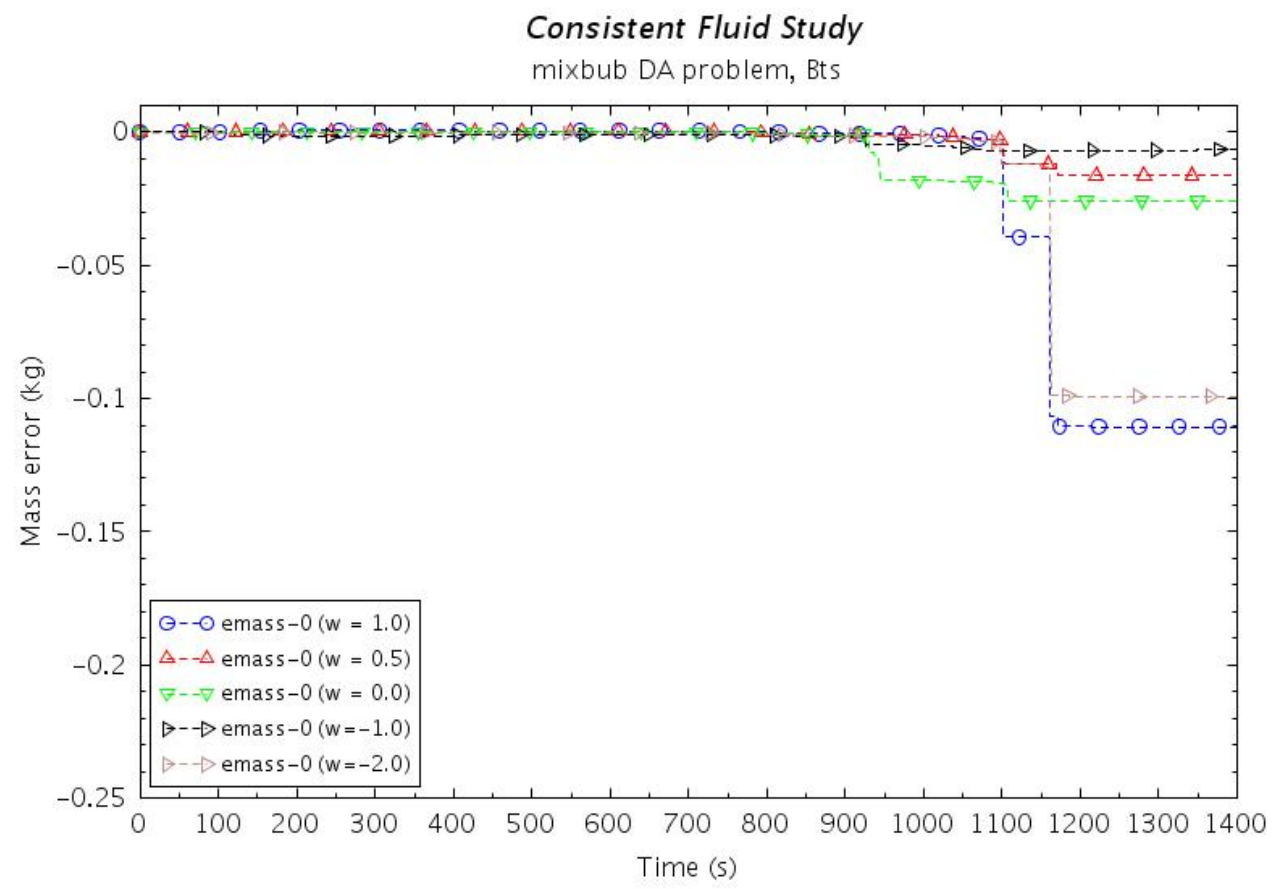

Figure 5.2.6.1b Mass error in mixbub.i for various omega values, BTS card-1 options

Comparison of Figs. 5.2.6.2a and 5.2.6.2b shows the following:

- Options $\boldsymbol{\omega}=0.0$ has the largest mass error in the INL case

- The default $\omega=1.0$ value is worst with the BTS options.

- In both cases $\boldsymbol{\omega}=-1$ is the best by far. These card-1 options have little effect on its mass error. 


\subsection{Conclusions}

RELAP5-3D has been updated to allow the code user to select the weighting factor, $\boldsymbol{\omega}$, for a hydrodynamic system containing $\mathrm{H} 2 \mathrm{ON}$. Various test cases, including simple insurge and outsurge problems, Edwards' pipe, typical PWR, Moby Dick, and mixbub, were evaluated with $\boldsymbol{\omega}$ values of $1.0,0.5,0.0,-1.0$, and -2.0 . No other weighting functions of other approximation methods for the fluid properties and their derivatives were considered. The evaluation showed that no particular value of $\boldsymbol{\omega}$ produced consistently better results in terms of mass error. The evaluation showed:

- Based on limited results, $\boldsymbol{\omega}$ also did not visibly affect engineering parameters such as pressure and void fraction.

- Crossing pressure and internal energy grid lines from one time advancement to the next affects mass error. In simple cases, pressure crossings cause local extrema and internal energy crossing inflect the curves. The effect diminishes as $\boldsymbol{\omega}$ tends toward zero, but is visible even for $\boldsymbol{\omega}=0.0$ for some problems.

- With the BTS options of Table 5.2.1, it is possible to generate TH property failures with typical PWR for all omega values at the largest DTMAX, as was shown in Fig. 5.2.4.2c and Table 5.2.4.

- The default value of $\boldsymbol{\omega}=1.0$ was seldom the best and sometimes the worst in terms of mass error and runtime.

- The value $\boldsymbol{\omega}=0.0$ was uniformly better for the simple insurge problems and was generally better for the outsurge problems, but was sometimes the worst with the more complicated input models.

- The value $\boldsymbol{\omega}=-1.0$ seldom performed poorly and performed the well or the best in a significant number of the more complicated cases. Therefore, $\boldsymbol{\omega}=-1.0$ of Eqn. (2-6) appears to be best option among the cases evaluated.

Although $\boldsymbol{\omega}=-1.0$ somewhat outperformed other choices and $\boldsymbol{\omega}=0.0$ performed well, neither excelled above the others significantly. That both methods match the linearity of the rest of the RELAP5-3D solution scheme indicates that linear approximation of fluid properties and their derivatives provides improvements in many cases due to improved consistency. From this limited testing, it is not clear that $\omega=-1.0$ or 0.0 is the better choice; nor is it clear that there may not be better means to approximate the fluid properties and their derivatives.

Additional test suites, such as the installation, developmental assessment, and others, could be run to further test the effects of $\boldsymbol{\omega}$ on mass error and robustness. 


\subsection{References}

1. The RELAP5-3D Code Development Team, "RELAP5-3D Code Manual Volume I: Code Structure, System Models and Solution Methods," INL-EXT-98-00834-V1, Revision 4.2, June, 2014.

2. C. B. Davis, "Interpolator Consistency Evaluation”, INL private correspondence, December, 2015.

3. The RELAP5-3D Code Development Team, "RELAP5-3D Code Manual Volume II, Appendix A: RELAP5-3D Input Requirements," INL-EXT-98-00834-V2, Revision 4.2, June, 2012.

4. G. L. Mesina, D. L. Aumiller, F. X. Buschman, "Extremely Accurate Sequential Verification of RELAP5-3D," Journal of Nuclear Science and Engineering, January, 2016.

5. C. B. Davis, "Accuracy Based Generation of Thermodynamic Properties for Light Water in RELAP5-3D," Rev. 1a, INL private correspondence, May, 2010.

6. A. R. Edwards and T. P. O'Brien, "Studies of Phenomena Connected with the Depressurization of Water Reactors," Journal of the British Nuclear Energy Society, pp. 125135, April 1970.

7. NEA/CSNI/R(97)3, "International Standard Problems (ISP), Brief Descriptions (1975 - 1997), Committee on the Safety of Nuclear Installations," OECD Nuclear Energy Agency, France, July 1997.

8. NEA/CSNI/R(2008)6/VOL2, "BEMUSE Phase IV Report: Simulation of a LB-LOCA in ZION Nuclear Power Plant, Appendices A to D," Committee on the Safety of Nuclear Installations, OECD Nuclear Energy Agency, France, November 2008.

9. The RELAP5-3D Code Development Team, "RELAP5-3D Code Manual Volume III, Developmental Assessment," INL-EXT-98-00834-V3, Revision 4.2, June, 2012. 